\title{
Determining fundamental parameters from the chargino sector in Left-Right Supersymmetric models
}

\author{
Nibaldo Alvarez-Moraga ${ }^{\dagger *}$ \\ $\dagger$ Autonomous Center of Theoretical Physics and Applied Mathematics, \\ 3561 Hutchison \# 3, Montréal (Québec) H2X 2G9, Canada
}

December 13, 2018

\begin{abstract}
Analytical expressions relating the fundamental parameters describing the chargino sector in the context of the Left-Right Supersymmetric model are constructed. A general complex extension of the real non-symmetric chargino mass matrix including all possible $\mathrm{CP}$-violating phases is considered. The method used for such a effects is the projector formalism based on the explicit knowledge of two unitary matrices diagonalizing the chargino mass matrix. Some possible scenarios allowing us to extract analytical and numerical values for the unknown parameters are considered. Moreover, an algorithm allowing us to disentangle the fundamental parameters of the chargino sector, based on possible measurements of some class of cross-section observables related to the chargino pair production in $e^{+} e^{-}$annihilation processes, is described. Some comparisons with the corresponding results in the context of the Minimal Supersymmetric Standard Model are given.
\end{abstract}

*Email address: nibaldo.alvarez.m@exa.pucv.cl 


\section{INTRODUCTION}

In the Left-Right Supersymmetric (L-R SUSY) model [1,2], which is based on the gauge group $S U(3)_{C} \times$ $S U(2)_{L} \times S U(2)_{R} \times U(1)_{B-L}$ [3], the masses and mixing matrices of the neutralinos and charginos are determined by $M_{L}, M_{R}$, the left-right gaugino mass parameters associated with the gauge group $S U(2)_{L}$ and $S U(2)_{R}$ respectively, $M_{V}$ the gaugino mass parameter associated with the gauge group $U(1)_{B-L}$, $\mu_{j}, j=1,2,3$, the Higgsino mass parameters, the ratio $\tan \theta_{k}=k_{u} / k_{d}$, where $k_{u}$ and $k_{d}$ are the vacuum expectation values $\left(V E V^{\prime} s\right)$ of the Higgs fields which couple to $d$-type and $u$-type quarks respectively, $v_{\Delta_{R}}$ the VEV of the Higgs triplet field $\Delta_{R}$ which together the triplet Higgs field $\Delta_{L}$ are associated to the spontaneous symmetry breaking of the group $S(2)_{R} \times U(1)_{B-L}$ to the hypercharge symmetry group $U(1)_{Y}$, and $v_{\delta_{R}}$, the VEV of the Higgs triplet field $\delta_{R}$ which together the triplet Higgs field $\delta_{L}$ are introduced to insure cancellation of the anomalies in the fermionic sector [4]-[10].

In Ref. [11] several relations connecting the parameters describing the neutralino sector in the context of the L-R SUSY model were described in terms of projectors, pseudoprojectors, reduced projectors and eigenphases[12, 13]. There, analytic expressions for the neutralino masses were obtained by diagonalizing the associated complex symmetric neutralino mass matrix. Then, based on the explicit knowledge of the corresponding diagonalizing unitary matrix novel formulae for the fundamental reduced projectors were constructed. Moreover, several CP-conserving and CP-violating possible scenarios were considered in the study of the so-called inverse problem consisting to express the fundamental parameters in terms of the neutralino physical masses, the eigenphases and the reduced projectors.

The purpose of this work is extending this procedure to study the exiting relations among the fundamental parameters describing the chargino sector of the L-R SUSY model. More precisely, we consider the terms in the total Lagrangian density which are relevant to describe the lightest chargino masses. The principal difference with the previous work is that in this case the chargino mass matrix is not symmetric and therefore one requires two different unitary matrices to diagonalize it. This implies the construction of two types of fundamental reduced projectors, one for each diagonalizing matrix, and the generalization of the method used in $[11,13]$ to treat the inverse problem. The study is carry out in a general context by introducing arbitrary $\mathrm{CP}$ violating phases in the mixing chargino matrix. Thus, we investigate, for instance, the effects of these phases on the chargino mass spectrum considering several CP-violating scenarios. Also, concerning the inverse problem, we study scenarios conditioning the values of these phases as well as the unknown fundamental parameters.

The paper is organized as follows, in Section 2, we write the Lagrangian density describing the light chargino sector in terms of the charged gaugino and Higgsino fields, the coupling constants associated to the gauge groups of the L-R SUSY model and the above mentioned fundamental parameters. According to our purpose, we write it in terms of the mixing chargino mass matrix. Also, in this section, in order to compare some of our results with the corresponding ones obtained in the context of the Minimal Supersymmetric Standard Model (MSSM), we describe briefly some aspects of this last model (for reviews see [14]).

In Section 3, we analyze numerically the chargino mass spectrum based on the analytical expressions for the chargino masses obtained by diagonalizing the above mentioned chargino mass matrix. The details 
of these calculus are given in Appendix A. We plot the masses versus the Higgsino mass parameter in some possible $\mathrm{CP}$-conserving and $\mathrm{CP}$-violating scenarios (effects of the $\mathrm{CP}$ violating phases on the chargino mass spectrum) and we compare the results with those obtained in [15] and also with those obtained using the corresponding formulas in the context of the MSSM [16]. In Section 4, we compute the analytical expressions for the corresponding diagonalizing unitary matrices.

In Section 5, the projector formalism [17] for this model is implemented in a original way. As in Ref. [11], we define the reduced projectors and eigenphases suitably and we express the entries of the diagonalizing chargino matrices in terms of them. Then, using an appropriate connection between the eigenvectors associated to these diagonalizing matrices we obtain a system of equations involving the fundamental parameters, the chargino masses, the eigenphases and the reduced projectors. We use these equations to express all the fundamental L-R SUSY parameters of the chargino sector in terms of the reduced projectors and eigenphases.

In Section 6, the disentangle of some fundamental parameters is considered. Using explicit expressions for the reduced projectors we disentangle the complex parameter $M_{L}$ from the chargino physical masses, the eigenphases and the rest of the parameters. Moreover, using the Jarlskog's formulas [17], we get an alternative formula to compute the norm of $M_{L}$ in terms of the last mentioned quantities, but with a given combination of the $\mathrm{CP}$-violating phases playing the role of the eigenphase arguments. In this section, we also propose an alternative parametrization based on some redefined reduced projectors and eigenphases allowing to disentangle the real parameter $M_{R}$ from the rest of the parameters, we compare with the corresponding case in the context of the MSSM. In Section 7 analytical and numerical reconstruction of the fundamental parameters is performed considering various possible $\mathrm{CP}$-conserving and $\mathrm{CP}$-violating scenarios.

In Section 8, based on some possible experimental measurements of cross-section-type observables related to the chargino pair production in $e^{+} e^{-}$annihilation processes a general algorithm for disentangle the fundamental parameters in the chargino sector is proposed. The general problem may be reduced to compute twelve independent parameters which can be chosen to be eight reduced projector norms (they can be parameterized by four pairs of spherical angles) and either four reduced projectors phases or four eigenphases. When the two lightest chargino masses are known the amount of independent parameters is reduced from twelve to ten. The expression for the parameter $\tan \theta_{k}$, in terms of the independent parameters and two lightest chargino masses is displayed in Appendix B.

Finally, in Appendix $\mathrm{C}$, in order to get a better overview of the complex interplay between the whole parameter set in that model, we construct some tables resuming the principal results of this paper.

\section{Chargino sector in Left-Right Supersymmetric model}

The particle content of the L-R SUSY model is different from that of the MSSM in the gauge sector, the Higgs sector and also having a right-handed neutrino in a weak isosinglet neutrino superfield. In this model, the gauge sector has an extra neutral $Z_{R}^{0}$ and two charged $W_{R}^{ \pm}$gauge bosons corresponding to the gauge 
group $S U(2)_{R}$. The Higgs sector contains two bi-doublet fields (in order to give masses to all the quarks)

$$
\phi_{u, d}=\left(\begin{array}{cc}
\phi_{1}^{0} & \phi_{1}^{+} \\
\phi_{2}^{-} & \phi_{2}^{0}
\end{array}\right)_{u, d}
$$

and four triplet fields,

$$
\Delta_{L, R}=\left(\begin{array}{cc}
\frac{1}{\sqrt{2}} \Delta^{+} & \Delta^{++} \\
\Delta^{0} & -\frac{1}{\sqrt{2}} \Delta^{+}
\end{array}\right)_{L, R}
$$

and

$$
\delta_{L, R}=\left(\begin{array}{cc}
\frac{1}{\sqrt{2}} \delta^{+} & \delta^{++} \\
\delta^{0} & -\frac{1}{\sqrt{2}} \delta^{+}
\end{array}\right)_{L, R}
$$

The Higgs $\phi_{u, d}$ both transform as $(1 / 2,1 / 2,0)$, and the Higgs $\Delta_{L, R}$ transform as $(1,0,2)$ and $(0,1,2)$, respectively. The triplet Higgs $\delta_{L, R}$ which transform as $(1,0,-2)$ and $(0,1,-2)$, respectively, are introduced to cancel anomalies in the fermionic sector that would otherwise occurs.

The full Lagrangian of the L-R SUSY model is given by [1]

$$
\mathcal{L}=\mathcal{L}_{\text {gauge }}+\mathcal{L}_{\text {matter }}+\mathcal{L}_{\mathrm{Y}}-\mathcal{V}+\mathcal{L}_{\text {soft }}
$$

where $\mathcal{L}_{\text {gauge }}$ contains the kinetic and self-interactions terms for the bosons vector fields $\left(W^{ \pm}, W^{0}\right)_{L, R}$ and $V^{0}$, and the Dirac Lagrangian of their corresponding superpartners, i.e., the gaugino fields $\left(\lambda^{ \pm}, \lambda^{0}\right)_{L, R}$ and $\lambda_{V}^{0} ; \mathcal{L}_{\text {matter }}$ contains the kinetic terms for the fermionic and bosonic matter fields, the Higgs fields and interaction of the gauge and matter multiplets; $\mathcal{L}_{Y}$ (Yukawa Lagrangian) contains the self-interaction terms of the matter multiplets, as well as the Higgs multiplets, e.g., it contains the self-interaction terms of the matter multiplets involving the fundamental Higgsino mass parameters $\mu_{1} \equiv \mu, \mu_{2}$ and $\mu_{3}: \operatorname{Tr}\left[\mu_{1}\left(\tau_{1} \tilde{\phi}_{u} \tau_{1}\right)^{T} \tilde{\phi}_{d}\right]$, $\operatorname{Tr}\left[\mu_{2}\left(\tau \cdot \tilde{\Delta}_{L}\right)\left(\tau \cdot \tilde{\delta}_{L}\right)\right]$ and $\operatorname{Tr}\left[\mu_{3}\left(\tau \cdot \tilde{\Delta}_{R}\right)\left(\tau \cdot \tilde{\delta}_{R}\right)\right]$, where $\tau_{j}, j=1,2,3$ are the usual Pauli matrices, $\tilde{\phi}_{u}$, $\tilde{\phi}_{d}, \tilde{\Delta}_{L, R}$ and $\tilde{\delta}_{L, R}$ are the superpartners of the bi-doublet field $\phi_{u}, \phi_{d}$ and the four triplet fields $\Delta_{L, R}$ and $\delta_{L, R}$, respectively; $\mathcal{V}$ is a scalar potential; $\mathcal{L}_{\text {soft }}$ is the soft-breaking Lagrangian, involving the fundamental gaugino mass parameters $M_{L}, M_{R}$ and $M_{V}$, which gives Majorana mass to the gauginos:

$$
\begin{aligned}
\mathcal{L}_{\text {soft }} & =M_{L}\left(\lambda_{L}^{a} \lambda_{L}^{a}+\bar{\lambda}_{L}^{a} \bar{\lambda}_{L}^{a}\right) \\
& +M_{R}\left(\lambda_{R}^{a} \lambda_{R}^{a}+\bar{\lambda}_{R}^{a} \bar{\lambda}_{R}^{a}\right) \\
& +M_{V}\left(\lambda_{V}^{0} \lambda_{V}^{0}+\bar{\lambda}_{V}^{0} \bar{\lambda}_{V}^{0}\right) .
\end{aligned}
$$

In order to generate mass for the gauge bosons we can choose the VEV's of the Higgs fields in the form [1]

$$
\begin{aligned}
\left\langle\Delta_{L}\right\rangle=\left\langle\delta_{L}\right\rangle=0, \quad\left\langle\Delta_{R}\right\rangle & =\left(\begin{array}{cc}
0 & 0 \\
& \\
v_{\Delta_{R}} & 0
\end{array}\right), \quad\left\langle\delta_{R}\right\rangle=\left(\begin{array}{cc}
0 & 0 \\
v_{\delta_{R}} & 0
\end{array}\right), \\
\left\langle\phi_{u}\right\rangle & =\left(\begin{array}{cc}
k_{u} & 0 \\
& \\
0 & 0
\end{array}\right), \quad\left\langle\phi_{d}\right\rangle=\left(\begin{array}{ll}
0 & 0 \\
& \\
0 & k_{d}
\end{array}\right) .
\end{aligned}
$$


Hence, the generation of mass for the gauge bosons can be analyzed in two separate stages of symmetry breaking. In the first stage, the break of $S U(2)_{R} \times U(1)_{B-L}$ into $U(1)_{Y}$, according to the VEV's of the $\Delta_{R}, \delta_{R}$ fields given in Eq. (2.6), generates masses for $W_{R}^{ \pm}, W_{R}^{0}$ and $V^{0}$. The two neutral states $W_{R}^{0}$ and $V^{0}$ mix yielding the physical field $Z_{R}$ and the massless field $B$. The vacuum expectation value $v_{\Delta_{R}}$ of the triplet Higgs $\Delta_{R}$ must be chosen big enough $\left(>>1 \mathrm{TeV}\right.$ ) to provide large masses to gauge bosons $W_{R}^{ \pm}$and $Z_{R}$. In the second stage, taking place at a much lower-energy scale, the spontaneous breaking of $S U(2)_{L} \times U(1)_{Y}$ into $U(1)_{\mathrm{em}}$, according the VEV's for $\phi_{u, d}$ given in Eq. (2.6), generates masses for the weak bosons $W_{L}^{ \pm}$ and $W_{L}^{0}$, as well as for $B_{\mu}$. Also in this stage, the masses for four light neutralinos $m_{\tilde{\chi}_{j}^{0}}, j=1, \ldots, 4$, and five charginos $m_{\tilde{\chi}_{j}^{ \pm}}, j=1, \ldots, 5$ are generated. Once again, the neutral fields mix forming the massless photon $A_{\mu}$ and the physical gauge field $Z_{L}$.

To find the chargino and neutralino masses, we consider the interaction terms between the gauge bosons, the Higgs, and their superpartners. In this way, for instance, the charged gaugino-higgsino mixing interaction Lagrangian is given by $[15,18]$

$$
\begin{aligned}
\mathcal{L}_{\text {chargino }} & =i v_{\Delta_{R}} g_{R} \tilde{\Delta}_{R}^{+} \lambda_{R}^{-}+i v_{\delta_{R}} g_{R} \tilde{\delta}_{R}^{-} \lambda_{R}^{+}+i g_{R} k_{d} \tilde{\phi}_{d 1}^{+} \lambda_{R}^{-}+i g_{L} k_{d} \bar{\phi}_{d 1}^{+} \lambda_{L}^{-}+i g_{R} k_{u} \tilde{\phi}_{u 2}^{-} \lambda_{R}^{+} \\
& +i g_{L} k_{u} \tilde{\phi}_{u 2}^{-} \lambda_{L}^{+}+\mu_{1} \tilde{\phi}_{u 1}^{+} \tilde{\phi}_{d 2}^{-}+\mu_{1} \tilde{\phi}_{u 2}^{-} \tilde{\phi}_{d 1}^{+}+M_{L} \lambda_{L}^{+} \lambda_{L}^{-}+M_{R} \lambda_{R}^{+} \lambda_{R}^{-} \\
& +\mu_{3} \tilde{\Delta}_{R}^{+} \tilde{\delta}_{R}^{-}+\mu_{3} \tilde{\Delta}_{R}^{++} \tilde{\delta}_{R}^{--}+H . C,
\end{aligned}
$$

where where $g_{L}$ and $g_{R}$ are the coupling constants of the gauge groups $S U(2)_{L}, S U(2)_{R}$, respectively; $\lambda_{L, R}^{ \pm}$are the $S U(2)_{L, R}$ gauginos fields, $\tilde{\phi}_{1 u}^{+}, \tilde{\phi}_{2 u}^{-}$and $\tilde{\phi}_{1 d}^{+}, \tilde{\phi}_{2 d}^{-}$the charged higgsino fields associated with the $u$ and $d$-quarks, respectively, and $\tilde{\Delta}_{R}^{+}, \tilde{\Delta}_{R}^{++}, \tilde{\delta}_{R}^{ \pm}, \tilde{\delta}_{R}^{--}$the charged right-handed higgsino fields.

As we have mentioned above, the VEVs $v_{\Delta_{R}}$ and $v_{\delta_{R}}$ are responsible for giving masses to $W_{R}$ and $Z_{R}$ bosons, as well as implementing the seesaw mechanism [3]. Thus, $v_{\Delta_{R}}$ is various orders greater than 1 $\mathrm{TeV}$ [19], i.e., we can consider, in a first approximation, the $\Delta_{R}^{+}$and $\delta_{R}^{-}$field decoupled from the lightest chargino states. The Lagrangian describing the lightest charginos can be written in the form

$$
\mathcal{L}_{\text {light-char. }}=-\frac{1}{2}\left(\begin{array}{ll}
\psi^{+^{T}} & \psi^{-T}
\end{array}\right)\left(\begin{array}{cc}
0 & M^{T} \\
M & 0
\end{array}\right)\left(\begin{array}{l}
\psi^{+} \\
\psi^{-}
\end{array}\right)+\text {h.c }
$$

where

$$
\begin{aligned}
& \psi^{+}=\left(-i \lambda_{L}^{+},-i \lambda_{R}^{+}, \tilde{\phi}_{u 1}^{+}, \tilde{\phi}_{d 1}^{+}\right)^{T}, \\
& \psi^{-}=\left(-i \lambda_{L}^{-},-i \lambda_{R}^{-}, \tilde{\phi}_{u 2}^{-}, \tilde{\phi}_{d 2}^{-}\right)^{T}
\end{aligned}
$$

and $M$ is the chargino mass matrix $\left(\mu_{1} \equiv \mu, \mu_{3}=0\right)$

$$
M=\left(\begin{array}{cccc}
M_{L} & 0 & 0 & \sqrt{2} \tilde{M}_{L} \cos \theta_{k} \\
0 & M_{R} & 0 & \sqrt{2} \tilde{M}_{R} \cos \theta_{k} \\
\sqrt{2} \tilde{M}_{L} \sin \theta_{k} & \sqrt{2} \tilde{M}_{R} \sin \theta_{k} & 0 & -\mu \\
0 & 0 & -\mu & 0
\end{array}\right),
$$

where we consider $M_{L}=\left|M_{L}\right| e^{i \Phi_{L}}, \mu=|\mu| e^{i \Phi_{\mu}}$,

$$
\tilde{M}_{L}=M_{W_{L}} e^{i \tilde{\Phi}_{L}}
$$


and

$$
\tilde{M}_{R}=\frac{g_{R}}{g_{L}} M_{W_{L}} e^{i \tilde{\Phi}_{R}},
$$

where $M_{W_{L}}$ denotes the mass of the left-handed gauge boson

$$
M_{W_{L}}=\frac{1}{\sqrt{2}} g_{L}\left(k_{u}^{2}+k_{d}^{2}\right)^{1 / 2} .
$$

Note that from Eq. (2.15) and the definition of $\theta_{k}, k_{u}$ and $k_{d}$ can be expressed in terms of $M_{W_{L}}, g_{L}$ and $\theta_{k}$ in the form

$$
\begin{aligned}
k_{u} & =\sqrt{2} \frac{M_{W_{L}}}{g_{L}} \sin \theta_{k}, \\
k_{d} & =\sqrt{2} \frac{M_{W_{L}}}{g_{L}} \cos \theta_{k} .
\end{aligned}
$$

In sum, in the CP-violating case, we assume that the lightest chargino mass matrix is parameterized by eight real parameters, namely, $\left|M_{L}\right|, \Phi_{L},|\mu|, \Phi_{\mu}, M_{R}, \tilde{\Phi}_{L}, \tilde{\Phi}_{R}$ and $\tan \theta_{k}$. On the other hand, in the $\mathrm{CP}$-conserving case, when all the phases are equal to zero, we assume that the chargino mass matrix is parameterized by four real parameters, $M_{L}, \mu, M_{R}$ and $\tan \theta_{k}$.

\subsection{Some aspects about the Minimal Supersymmetric Standard Model}

Along this work it will be particulary interesting to compare the results of some physical quantities obtained from the LR-SUSY model with those obtained from the MSSM, which is based on the gauge group $S U(3)_{C} \times S U(2)_{L} \times U(1)_{Y}$. The particle content of the MSSM are the one of the Standard Model plus the corresponding superpartners, but besides that, the MSSM includes two complex scalar Higgs doublets field $H_{u}=\left(H_{u}^{+}, H_{u}^{0}\right)$ and $H_{d}=\left(H_{d}^{0}, H_{d}^{-}\right)$plus partners and a singlet partner field $\psi_{x}$ and $\tilde{\psi}_{x}$. The break of electroweak symmetry down to electromagnetism $S U(2)_{L} \times U(1)_{Y} \mapsto U(1)$, is realized by giving nonzero VEVs to the Higgs fields $\left\langle H_{u}^{0}\right\rangle=v_{u}$ and $\left\langle H_{u}^{0}\right\rangle=v_{d}$. These VEVs can be connected to the known mass of the $Z^{0}$ boson and the electroweak gauge couplings $g, g^{\prime}$ :

$$
v_{u}^{2}+v_{d}^{2}=\frac{2 m_{Z}^{2}}{\left(g^{2}+g^{\prime 2}\right)} \approx(174 \mathrm{GeV})^{2} .
$$

The ratio of the two VEVs is written as $\tan \beta=v_{u} / v_{d}$. Among the effects of electroweak symmetry breaking, the neutral higgsinos $\tilde{H}_{u}^{0}$ and $\tilde{H}_{d}^{0}$ and the neutral gauginos $\tilde{B}$ and $\tilde{W}^{0}$ combine to form four neutral mass eigenstates, i.e., the neutralinos. The charged higgssinos $\tilde{H}_{u}^{+}$and $\tilde{H}_{d}^{-}$and winos $\tilde{W}^{+}$and $\tilde{W}^{-}$mix to form two mass eigenstates with charge \pm 1 called charginos. Thus, the mass content of the neutralino sector is described by a $4 \times 4$ symmetric matrix which, in the most general CP-violating case[13], is parameterized by two complex quantities, $M_{1}=\left|M_{1}\right| e^{i \Phi_{1}}$ and $\mu=e^{i \Phi_{\mu}}$, (the supersymmetric higssino mass parameter) and two real quantities, $M_{2}$ (the $S U(2)$ gaugino mass), and $\tan \beta=v_{2} / v_{1}$. Here, the phase angles are defined such that $\pi \leq \Phi_{1} \leq \pi$ and $\pi \leq \Phi_{\mu} \leq \pi$. The chargino mass matrix is given by the $2 \times 2$ non-symmetric matrix

$$
\mathcal{M}_{C}=\left(\begin{array}{cc}
M_{2} & \sqrt{2} m_{W} \cos \beta \\
\sqrt{2} m_{W} \sin \beta & \mu
\end{array}\right) \text {, }
$$


where $m_{W} \approx 80,419 \mathrm{GeV}$ is the mass of the vector boson gauge field $W$. Introducing a CP-violating phase in the higgsino parameter $\mu=|\mu| e^{i \Phi_{\mu}}$, we can show that the chargino mass spectrum for this model is given by [20]

$$
m_{\tilde{\chi}_{1,2}^{ \pm}}^{2}=\frac{1}{2}\left(M_{2}^{2}+|\mu|^{2}+2 m_{W}^{2} \mp \Delta_{C}\right)
$$

where

$$
\Delta_{C}=\sqrt{\left(M_{2}^{2}-|\mu|^{2}\right)^{2}+4 m_{W}^{4} \cos ^{2}(2 \beta)+4 m_{W}^{2}\left(M_{2}^{2}+|\mu|^{2}\right)+8 m_{W}^{2} M_{2}|\mu| \sin (2 \beta) \cos \Phi_{\mu}} .
$$

The parameter determination problem in both cases, the CP-conserving and CP-violating, has been studied in detail in the literature, see for example [13, 20, 23, 24].

\section{Chargino masses, numerical results}

In this section we investigate the chargino mass spectrum of the LR-SUSY under some chosen CP-conserving and CP-violating scenarios we compare the results with those obtained in similar conditions in the context of the MSSM.

The physical chargino mass eigenstates are related to the states given in Eqs. (2.10) and (2.11) as

$$
\begin{array}{ll}
\psi_{i}^{+}=\sum_{j=1}^{4} V_{i j} \chi_{j}^{+}, & i=1, \ldots, 4, \\
\psi_{i}^{-}=\sum_{j=1,4} U_{i j} \chi_{j}^{-} & i=1, \ldots, 4,
\end{array}
$$

respectively, where the unitary $U$ and $V$ matrices satisfies

$$
\begin{aligned}
M_{D} & =U^{T} M V, \\
& \equiv \sum_{j=1}^{4} m_{\tilde{\chi}_{j}^{ \pm}} E_{j},
\end{aligned}
$$

and

$$
\begin{aligned}
M_{D}^{2} & =V^{-1} M^{\dagger} M V=U^{T} M M^{\dagger} U^{*} \\
& \equiv \sum_{j=1}^{4} m_{\tilde{\chi}_{j}^{ \pm}}^{2} E_{j},
\end{aligned}
$$

where $\left(E_{j}\right)_{4 \times 4}$ are the basic matrices defined by

$$
\left(E_{j}\right)_{i k}=\delta_{j i} \delta_{j k}
$$

Here, we suppose that the real eigenvalues of $M_{D}$ are ordered in the following way

$$
m_{\tilde{\chi}_{1}^{ \pm}} \leq m_{\tilde{\chi}_{2}^{ \pm}} \leq m_{\tilde{\chi}_{3}^{ \pm}} \leq m_{\tilde{\chi}_{4}^{ \pm}}
$$




\begin{tabular}{ccccc}
\hline Scenario & $M_{R}$ & $M_{L}$ & $M_{W_{L}}$ & $\tan \theta_{k}$ \\
\hline$S c p c_{1}$ & 300 & 50 & 50.271 & 1.6 \\
\hline$S c p c_{2}$ & 200 & 150 & 80.456 & 3 \\
\hline
\end{tabular}

Table 1: Input parameters for scenarios $S c p c_{1}$ and $S c p c_{2}$. All mass quantities are given in GeV.

The resulting diagonal matrix $M_{D}$, containing the chargino masses, and the matrices $U$ and $V$ are known only for the CP-conserving case under the assumptions that $g_{L} \approx g_{R}$ and in limit of large $M_{R}, M_{L}$ or $\mu$,

$$
\left|M_{L} \mu\right| \gg \tilde{M}_{L}^{2} \sin ^{2} \theta_{k}, \quad\left|M_{R} \mu\right| \gg \tilde{M}_{R}^{2} \sin ^{2} \theta_{k}
$$

and similarly for $\sin ^{2} \theta_{k} \leftrightarrow \cos ^{2} \theta_{k}$ [15]. In Appendix A, we put into practice a method [11, 12, 13, 21, 22] giving analytic expressions for these masses without limiting the values of the parameters. In this way, in the most general case, we directly observe that to determine the chargino masses in the context of L-R SUSY model we must to fix eight real parameters, namely $\left|M_{L}\right|, \Phi_{L},|\mu|, \Phi_{\mu}, M_{R}, \tilde{\Phi}_{L}, \tilde{\Phi}_{R}$, and $\tan \theta_{k}$, whereas in the MSSM, the chargino masses only depend on four real fundamental parameters, $M_{2},|\mu|, \Phi_{\mu}$ and $\tan \beta$.

\subsection{CP-conserving scenarios}

Let us consider the CP-conserving scenarios $S c p c_{1}$ and $S c p c_{2}$ described in Tab. 1. These scenarios are similar to the ones studied in Ref. [15] where they have been used to compare the chargino masses predicted by the L-R SUSY model and the MSSM. For both scenarios, we consider the coupling constants values $g_{R} \approx g_{L}=0.65$. To make suitable the general results given in Eqs. (A.12) and (A.13) to these situations we must take in Eqs. (A.9-A.11) the mixing phases $\Phi_{L}=\tilde{\Phi}_{L}=\tilde{\Phi}_{R}=0$ and $\Phi_{\mu}=0, \pi$.

Figures 1 and 2, show the behaviour of the chargino physical masses $m_{\tilde{\chi}_{i}^{ \pm}}, i=1, \ldots, 4$, versus $\mu$, computed from Eqs. (A.12-A.13), for input parameters of scenarios $S c p c_{1}$ and $S c p c_{2}$, respectively. We observe the correct size ordering of the chargino masses, such as required by Eq. (3.27). Also, in both scenarios, we find that for $|\mu| \sim 200 \mathrm{GeV}$ the chargino masse $m_{\tilde{\chi}_{1}^{ \pm}}$is equal to $M_{L}$, approximately, and for $|\mu| \sim 300 \mathrm{GeV}$ the the chargino masse $m_{\tilde{\chi}_{3}^{ \pm}}$, is of the order of $M_{R}$. On the other hand, considering the values of the chargino masse $m_{\tilde{\chi}_{4}^{ \pm}}$, we observe that for all values of $|\mu|$ they are always greater than $M_{R}$.

In Fig. 1, we observe that the mass $m_{\tilde{\chi}_{1}^{ \pm}}=|\mu|$, when $-20 \mathrm{GeV} \lesssim \mu \lesssim 60 \mathrm{GeV}$ and $m_{\tilde{\chi}_{2}^{ \pm}}=|\mu|$, in the complementary region, whereas in Fig. 2 we observe that the mass $m_{\tilde{\chi}_{2}^{ \pm}}=|\mu|$, when $-175 \mathrm{GeV} \lesssim$ $\mu-\lesssim 25 \mathrm{GeV}$ and $125 \mathrm{GeV} \lesssim \mu \lesssim 185 \mathrm{GeV}, m_{\tilde{\chi}_{1}^{ \pm}}=|\mu|$, when $-25 \mathrm{GeV} \lesssim \mu \lesssim 125 \mathrm{GeV}$ and $m_{\tilde{\chi}_{3}^{ \pm}}=|\mu|$, in the complementary region. This is an important feature that we must taking into account when we study the inverse problem. Indeed, we can show that $|\mu|^{2}$ is an exact solution of the characteristic equation determining the chargino spectrum (in either CP-conserving or CP-violating case) (see Appendix 


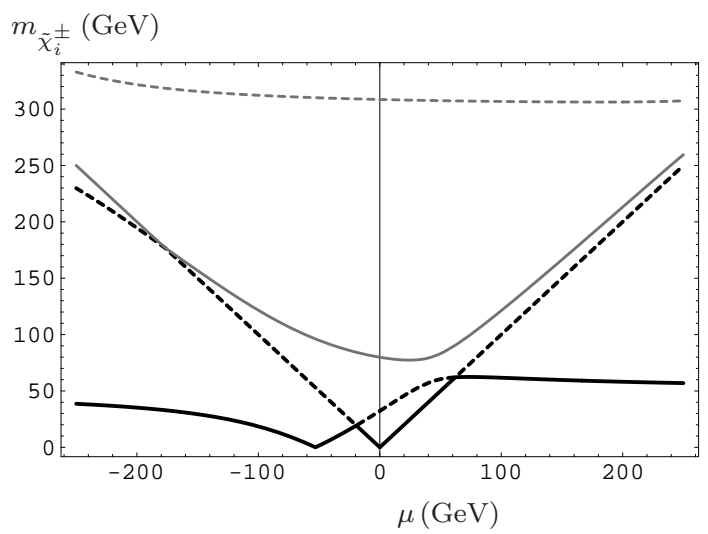

Figure 1: chargino masses $m_{\tilde{\chi}_{j}^{ \pm}}, i=1, \ldots, 4$, as functions of $\mu$ for scenario input parameters of scenario $S c p c_{1}$.

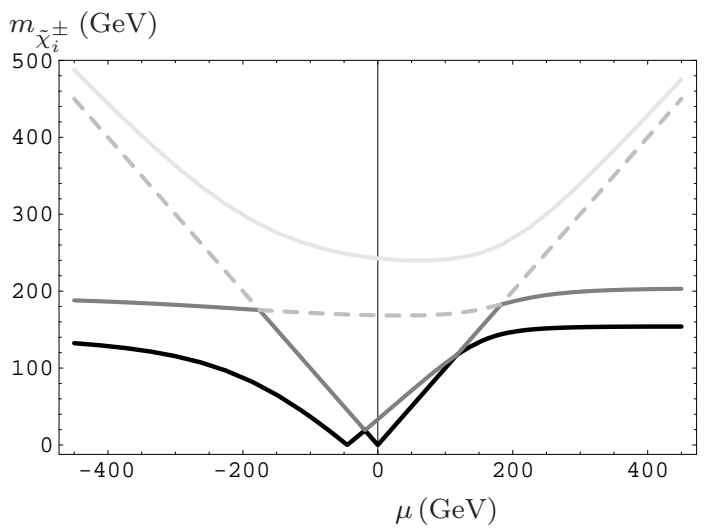

Figure 2: chargino masses $m_{\tilde{\chi}_{j}^{ \pm}}, j=1, \ldots, 4$, as functions of $\mu$ for input parameters of scenario $S c p c_{2}$.

A), i.e., it is always possible to find a region in the fundamental parameter space where one of the chargino masses exactly takes the value $|\mu|$.

\subsection{CP-violating scenarios}

In this section we study the effects of the CP-violating phases on the chargino mass spectrum. Also, we analyze the singularities in the curves $m_{\tilde{\chi}_{1}^{ \pm}}$versus $\mu$ and we compare with the corresponding ones obtained using the formulas for the MSSM. Moreover, we study the behaviour of this mass when $\tan \theta_{k}$ varies and again we compare with the results obtained in the context of the MSSM.

\subsubsection{Chargino mass spectrum when varying $\mu$ and the phase angles}

Let us now to study the effects produced by the variation of the mixing phases on the curves describing the behaviour of the chargino masses as a function of $\mu$. Let us consider, for instance, the chargino masses $m_{\tilde{\chi}_{i}^{ \pm}}, i=1,2$, given in Eq. (A.12) and the CP-violating scenario $S c p v_{1}$ described in Tab. 2. Figure 3 shows the effects produced by the variation of the phase $\Phi_{L}$ on the curves describing the chargino mass $m_{\tilde{\chi}_{1}^{ \pm}}$as a 


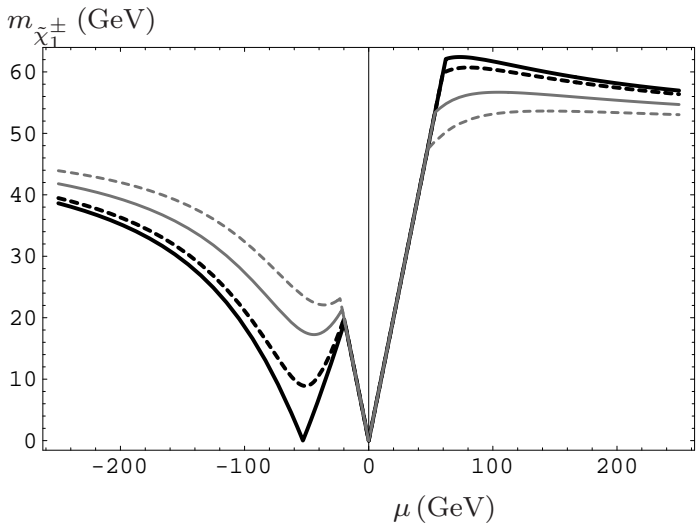

Figure 3: Chargino masse $m_{\tilde{\chi}_{1}^{ \pm}}$as a function of $\mu$ for scenario $S c p v_{1}$, assuming $\tilde{\Phi}_{L}=\tilde{\Phi}_{R}=0$. The curves are: $\Phi_{L}=0$ (heavy solid), $\Phi_{L}=\pi / 8$ (heavy dashed), $\Phi_{L}=\pi / 4$ (light solid), $\Phi_{L}=\pi / 3$ (light dashed).

function of $\mu$, for input parameters of scenario $S c p v_{1}$ with fixed phases $\tilde{\Phi}_{L}=\tilde{\Phi}_{R}=0$. We observe that for values of $-20 \mathrm{GeV} \lesssim \mu \lesssim 60 \mathrm{GeV}$, there is not difference between the curves with $\Phi_{L}=0$ and those with $\Phi_{L}=\pi / 8, \pi / 4, \pi / 3$. However, for values of $\mu \lesssim-20 \mathrm{GeV}$ or $\mu \gtrsim 60 \mathrm{GeV}$, we observe for some values of $\mu$, differences attaining as far as $6 \mathrm{Gev}$ approximately. Also, we observe that in these cases the differences with respect to the variation of $\Phi_{L}$ are positives when $\mu \lesssim-20 \mathrm{GeV}$ and negatives when $\mu \gtrsim 60 \mathrm{GeV}$.

The same arguments are valid when we consider the effects produced by the variation of the phase $\tilde{\Phi}_{L}$ on these curves, as we can see by observing Fig. 4. However, in this last case, the differences between the curves for some values of $\mu$ can attain as far as $16 \mathrm{Gev}$ and the lower limit of positif values of $\mu$ where some differences are detected is $30 \mathrm{Gev}$, approximately. On the other hand, if we consider the effect on the curves induced by the variation of the phase $\tilde{\Phi}_{R}$, we don't appreciate important differences among them. Indeed, for positif values of $\mu$ there is none. Contrarily to the above analysis, for the curves describing the chargino mass $m_{\tilde{\chi}_{2}^{ \pm}}$as a function of $\mu$, the intervals where we appreciate differences among the curves which are consequences of the variation of the phases $\Phi_{L}, \tilde{\Phi}_{L}$ or $\tilde{\Phi}_{R}$ are practically inverted.

Note that the irregularities in the plots of Figs. 3 and 4, produces because the curve representing the variation with respect to $\mu$ of the factor $\sqrt{\beta-\bar{w}-\lambda / 4 \alpha}$ in Eq. (A.13), take the forme of a $\mathcal{W}$, approxi-

\begin{tabular}{cccccc}
\hline Scenario & $\Phi_{L}, \tilde{\Phi}_{L}, \tilde{\Phi}_{R}$ & $M_{R}$ & $\left|M_{L}\right|$ & $k_{u}$ & $\tan \theta_{k}$ \\
& 0 & & & & \\
& $\pi / 8$ & & & & \\
$S_{c p v v_{1}}$ & $\pi / 4$ & 300 & 50 & 92.75 & 1.6 \\
& $\pi / 3$ & & & & \\
& & & & & \\
\hline
\end{tabular}

Table 2: Input parameters for scenario $S c p v_{1}$. All mass quantities are given in GeV. 


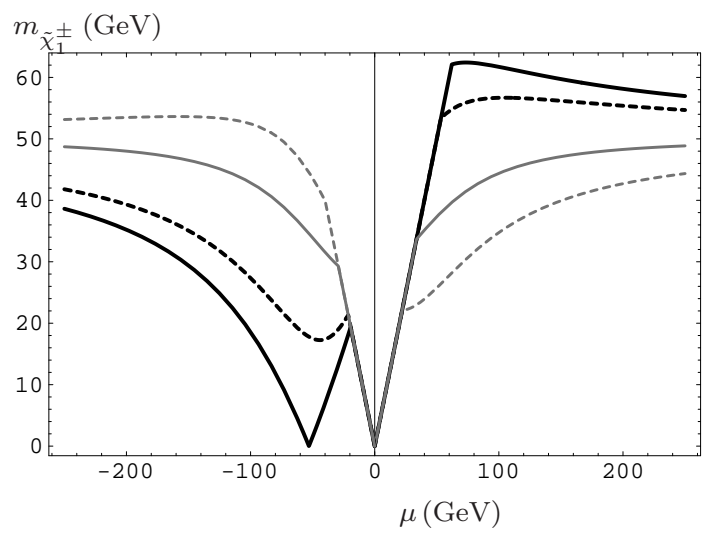

Figure 4: Chargino masse $m_{\tilde{\chi}_{1}^{ \pm}}$as a function of $\mu$ for scenario $S c p v_{1}$, assuming $\Phi_{L}=\tilde{\Phi}_{R}=0$. The curves are: $\tilde{\Phi}_{L}=0$ (heavy solid), $\tilde{\Phi}_{L}=\pi / 8$ (heavy dashed), $\tilde{\Phi}_{L}=\pi / 4$ (light solid), $\tilde{\Phi}_{L}=\pi / 3$ (light dashed).

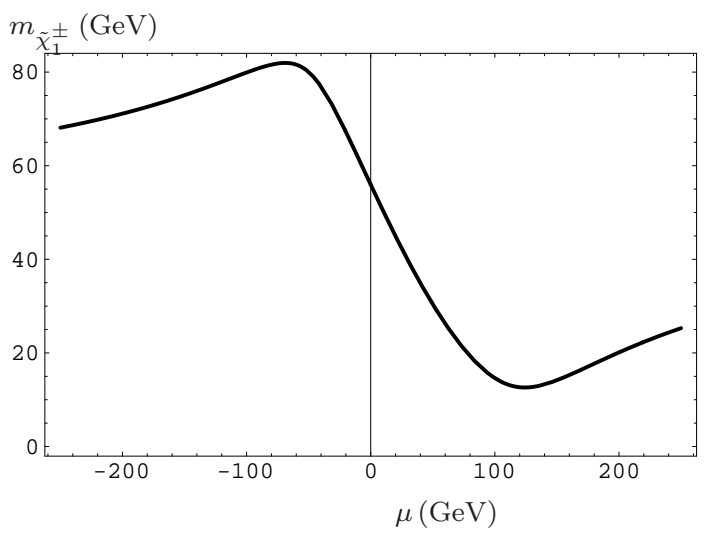

Figure 5: Chargino masse $m_{\tilde{\chi}_{1}^{ \pm}}$as a function of $\mu$, computed from Eq. (2.20) in the context of the MSSM, assuming $m_{W}=80.419 \mathrm{GeV}, M_{2}=50 \mathrm{GeV}$ and $\tan \beta=1.6$. 
mately, whereas the curve representing the variation with respect to $\mu$ of the factor $a / 4-\alpha / 2$ in that equation take the forme of a hyperbolic branch, approximately, both curves with the ordinate-axis as its symmetry axis. The hyperbolic branch being inscribed in the $\mathcal{W}$ curve approach asymptotically to this one for positive values of $\mu$ and go away from this one, after practically intersect it, for negative values of $\mu$. Thus, in this case, we can say that the change in the mixing character is determined by the factor $\sqrt{\beta-\bar{w}-\lambda / 4 \alpha}$ and tuned and displaced by the factor $a / 4-\alpha / 2$. In the corresponding case of the MSSM, the tuning factor in Eq. (2.20) take the form of a parabola and the change in the mixing character, determined in combination with the factor $\Delta_{C} / 2$, is smooth, see Fig 5. We don't observe an important displacement of the curve with respect to the ordinate-axis as in the case of the LR-SUSY model. Also, for large values of $|\mu|$, we observe an inverted mixing behaviour with respect to the one observed in the L-R SUSY model.

\subsubsection{Chargino mass $m_{\tilde{\chi}_{1}^{ \pm}}$as a function of $\tan \boldsymbol{\theta}_{\boldsymbol{k}}$}

\begin{tabular}{lcccccc}
\hline Scenario & $\Phi_{L}=\tilde{\Phi}_{L}=\tilde{\Phi}_{R}$ & $M_{R}$ & $\left|M_{L}\right|$ & $|\mu|$ & $M_{W_{L}}=m_{W}$ & $M_{2}$ \\
\hline$S c p v_{2}$ & 0 & 300 & 152 & 248 & 80.456 & 152 \\
\hline
\end{tabular}

Table 3: Input parameters for scenario $\mathrm{Scpv}_{2}$. All mass quantities are given in GeV.

In this section we investigate the behaviour of the lightest chargino mass when the ratio $k_{u} / k_{d}=\tan \theta_{k}$ varies, and we compare with the behaviour of this mass in the context of the MSSM when $\tan \beta$ is allowed to vary.

Let us consider the scenario $S c p v_{2}$, described in Tab. 3, where in addition to the the input parameters for the L-R SUSY model, we include the input parameters representing similar conditions in the context of the MSSM. Figure 6 shows the curves describing the variation of the chargino mass $m_{\tilde{\chi}_{1}^{ \pm}}$(in the context of the L-R SUSY model) with respect to $\tan \theta_{k}$ for input parameters of scenario $S c p v_{2}$ when $\Phi_{\mu}=0, \pi / 4, \pi / 2,3 \pi / 4, \pi$ (the curves are distingued by its grey level, from the darkest one $\left(\Phi_{\mu}=0\right)$ to the lightest one $\left(\Phi_{\mu}=\pi\right)$, respectively). We observe that, for values of $0 \leq \Phi_{\mu}<\pi / 2$, the chargino mass decrease when $\tan \theta_{k}$ increase whereas for values of $\pi / 2<\Phi_{\mu} \leq \pi$, there is a change of the mixing character, i.e., the chargino mass increase when $\tan \theta_{k}$ grows. For small values of $\Phi_{\mu}$ and $\theta_{k}$, the chargino mass $m_{\tilde{\chi}_{1}^{ \pm}}$is of order of $\left|M_{L}\right|$. For large values of $\tan \theta_{k}$ and for all $\Phi_{\mu}$, the values of $m_{\tilde{\chi}_{1}^{ \pm}}$approach to the common value $\approx 136 \mathrm{GeV}$, i.e., the value corresponding to the phase angle $\Phi_{\mu}=\pi / 2$. In general, in this scenario the value of $m_{\tilde{\chi}_{1}^{ \pm}}$lies in the range $112 \mathrm{GeV}-152 \mathrm{GeV}$.

Figure 7 shows curves describing the variation of the chargino mass $m_{\tilde{\chi}_{1}^{ \pm}}$(in the context of the MSSM) with respect to $\tan \beta$ for input parameters of scenario $S c p v_{2}$, when $\Phi_{\mu}=0, \pi / 4, \pi / 2,3 \pi / 4, \pi$ ( again, the curves are distinguished by its grey level, from the darkest one $\left(\Phi_{\mu}=0\right)$ to the lightest one $\left(\Phi_{\mu}=\pi\right)$, respectively). Comparing these curves with those of the LR-SUSY model, we observe an opposite change 


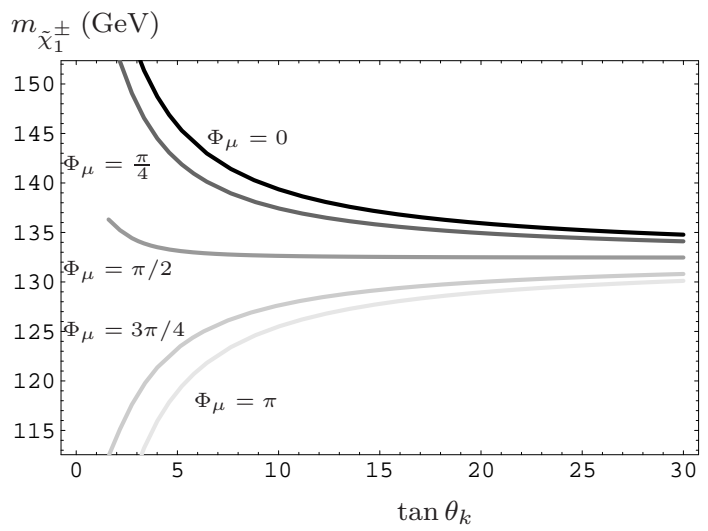

Figure 6: Chargino mass $m_{\tilde{\chi}_{1}^{ \pm}}$as a function of $\tan \theta_{k}$ for input parameters of scenario $S c p v_{2}$, in the context of LR-SUSY model.

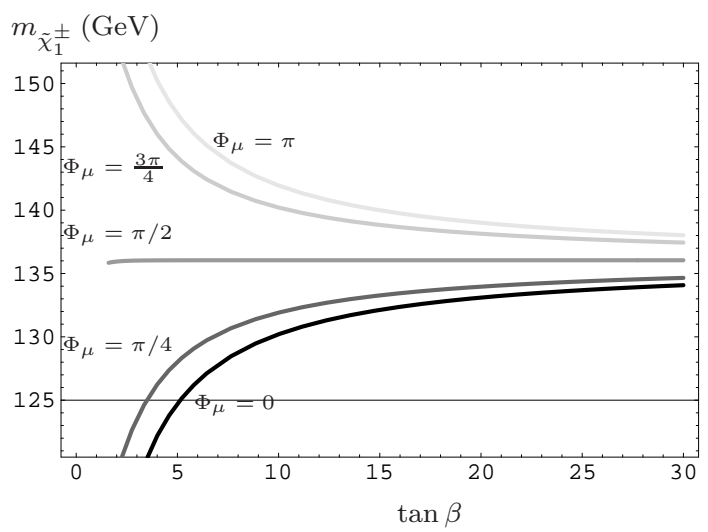

Figure 7: Chargino mass $m_{\tilde{\chi}_{1}^{ \pm}}$as a function of $\tan \beta$ for input parameters of scenario $S c p v_{2}$, in the context of MMSM.

rate of the chargino mass $m_{\tilde{\chi}_{1}^{ \pm}}$with respect to $\tan \beta$, approximately, into the same intervals of variation of $\Phi_{\mu}$ described in the previous paragraph. This last fact illustrates the way as the parameter $\Phi_{\mu}$ provides of an inverted mixing character to both models (a phase difference of $\pi$, approximately ).

In the MSSM the order of magnitude of the chargino mass $m_{\tilde{\chi}_{1}^{ \pm}}$is dominated by the parameter $M_{2}$, whereas in the LR-SUSY model this magnitude is determined by both the $\left|M_{L}\right|$ and $M_{R}$ parameters. For instance, if we take $M_{R}=100 \mathrm{GeV}$ in place of $300 \mathrm{GeV}$ in Tab. 3, then we find that the values of $m_{\tilde{\chi}_{1}^{ \pm}}$lies in the range $70 \mathrm{GeV}-110 \mathrm{GeV}$.

\section{Construction of the unitary $U$ and $V$ matrices}

The diagonalizing matrix $V$ can be obtained by computing the eigenvectors $v_{j}$ of $H \equiv M^{\dagger} M$, corresponding to the eigenvalues $m_{\tilde{\chi}_{j}^{ \pm}}$given in Eqs. (A.12) and (A.13):

$$
v_{j}=\left(\begin{array}{llll}
V_{1 j} & V_{2 j} & V_{3 j} & V_{4 j}
\end{array}\right)^{T}, \quad j=1, \ldots, 4 .
$$


Following the results in Ref.[11], we get the $V_{i j}$ entries

$$
V_{i j}=\frac{\Delta_{i j}}{\Delta_{1 j}} \frac{\left|\Delta_{1 j}\right| e^{i \theta_{j}}}{\sqrt{\left|\Delta_{1 j}\right|^{2}+\left.\left.\left|\Delta_{2 j}\right|^{2}|+| \Delta_{3 j}\right|^{2}|+| \Delta_{4 j}\right|^{2}}},
$$

when $\mathrm{i}=1, \ldots, 4$. Here

$$
\Delta_{1 j}(H)=\left|\begin{array}{ccc}
H_{22}-m_{\tilde{\chi}_{j}^{ \pm}}^{2} & H_{23} & H_{24} \\
H_{32} & H_{33}-m_{\tilde{\chi}_{j}^{ \pm}}^{2} & H_{34} \\
H_{42} & H_{43} & H_{44}-m_{\tilde{\chi}_{j}^{ \pm}}^{2}
\end{array}\right|
$$

and $\Delta_{i j}(H), i=2,3,4$, is formed from $\Delta_{1 j}(H)$ by substituting the $(i-1)$ th column by $\left(\begin{array}{l}-H_{21} \\ -H_{31}\end{array}\right)$. In the same way, the eigenvectors $u_{j}^{*}$ forming the $U^{*}$ matrix are given by

$$
u_{j}^{*}=\left(\begin{array}{llll}
U_{1 j}^{*} & U_{2 j}^{*} & U_{3 j}^{*} & U_{4 j}^{*}
\end{array}\right)^{T}, \quad j=1, \ldots, 4,
$$

where the $U_{i j}^{*}$ matrix's elements are given by

$$
U_{i j}^{*}=\frac{\tilde{\Delta}_{i j}}{\tilde{\Delta}_{1 j}} \frac{\left|\tilde{\Delta}_{1 j}\right| e^{-i \tilde{\theta}_{j}}}{\sqrt{\left|\tilde{\Delta}_{1 j}\right|^{2}+\left.\left.\left|\tilde{\Delta}_{2 j}\right|^{2}|+| \tilde{\Delta}_{3 j}\right|^{2}|+| \tilde{\Delta}_{4 j}\right|^{2}}},
$$

when $\mathrm{i}=1, \ldots, 4$. Here, $\tilde{\Delta}_{i j}(\tilde{H}) \equiv \Delta_{i j}(\tilde{H}), i, j=1, \ldots, 4$.

At this point it is pertinent to clarify that considering both the particular values taken by the entries of the $H$ matrix, namely $H_{31}=H_{32}=H_{34}=0$ and $H_{33}=|\mu|^{2}$, and the particular value taken by one of the physical chargino masses $m_{\tilde{\chi}_{j}^{ \pm}}=|\mu|$, when $j=1,2,3$ or 4 , (see Appendix A), so for a given $j$ the corresponding $\Delta_{i j}, i=1,2, \ldots, 4$, factors defined in Eq. (4.31) become zero, i.e., the $V$ matrix becomes singular. This fact obliges us to pay special attention at the moment to compute the eigenvector associated to this particular chargino mass value. Indeed, the usual theory of matrix eigenvalues and eigenvectors provides us the tools we need to remedy this situation. The same is valid for the $\tilde{\Delta}_{i j}$ factors. Hereafter we suppose, without any loss of generality (concerning the general method) that we work in a region of the fundamental parameter space where $m_{\tilde{\chi}_{3}^{ \pm}}=|\mu|$. For other regions where a different chargino has mass equal to $|\mu|$, the subsequent analysis is modified only slightly to the extent that role of the old $j=3$ index is played by the new $j$ index associated to the new fixed chargino mass. The general formulation that we present here allows us to do it without any problem.

Continuing with our arguments, both the phases $\theta_{j}$ 's in Eq. (4.30) and the phases $\tilde{\theta}_{j}$ 's in Eq. (4.33) are arbitrary, they will be fixed by the requirement that $U$ and $V$ satisfy Eq. (3.24). As we will see in the next section, these phases are related to the so-called CP eigenphases [11, 13].

On the other hand, as $v_{j}$ is an eigenvector of $H$ associated to the eigenvalue $m_{\tilde{\chi}_{j}^{ \pm}}$, i.e.,

$$
M^{\dagger} M v_{j}=m_{\tilde{\chi}_{j}^{ \pm}} v_{j},
$$

then multiplying both sides of this equation by $M$, we get

$$
M M^{\dagger}\left(M v_{j}\right)=m_{\tilde{\chi}_{j}^{ \pm}}\left(M v_{j}\right),
$$


i.e, $M v_{j}$ is an eigenvector of $\tilde{H}$ corresponding to the same eigenvalue $m_{\tilde{\chi}_{j}^{ \pm}}$. Thus, according to Eq. (3.25) and taking in account the unitary character of $U^{*}$, we can show that the normalized eigenvectors $u_{j}^{*}$ forming this matrix are given by

$$
u_{j}^{*}=\frac{1}{m_{\tilde{\chi}_{j}^{ \pm}}} M v_{j},
$$

when $j=1, \ldots, 4$. Thus, substituting Eq. (4.29) in Eq. (4.36) and comparing with Eq. (4.32), we get

$$
U_{i j}^{*}=\frac{1}{m_{\tilde{\chi}_{j}^{ \pm}}} \sum_{k=1}^{4} M_{i k} V_{k j} .
$$

Equations (4.33) and (4.37) represent two equivalent ways to write the entries of the $U^{*}$ matrix.

On the other hand, by the same arguments we get

$$
V_{i j}=\frac{1}{m_{\tilde{\chi}_{j}^{ \pm}}} \sum_{k=1}^{4} M_{i k}^{\dagger} U_{k j}^{*} .
$$

Thus, equations (4.30) and (4.38) represent two equivalent ways to write the entries of the $V$ matrix.

\section{The chargino projectors, reduced projectors, pseudoprojectors and CP eigenphases}

In this section we show how to implement the projector formalism [17] to describe the chargino observables in the L-R SUSY model.

It has been demonstrated that the projector formalism is very efficient to describe the neutralino observables in both the the MSSM [13] and the L-R SUSY model [11], where the neutralino mass matrix was described by a real or complex $4 \times 4$ symmetric matrix. So in both, the $\mathrm{CP}$-conserving and CP-violating cases, this formalism represents a systematic method to express the fundamental parameters of the neutralino sector in terms of some basic quantities, namely the reduced projectors and eigenphases. Conversely, these last quantities can be expressed in terms of the fundamental parameters and physical masses in a direct manner. Other important characteristic of this formalism is that it can be easily generalized to any neutralino number.

Concerning the chargino sector, in the MSSM we must deal with a $2 \times 2$ non-symmetric matrix. The problem of determining the fundamental chargino parameters including all possible CP-violating phases into the chargino mixing matrix has been completely solved by standard diagonalization matrix methods, see for instance $[19,20]$ an references therein. In the case of the LR-SUSY model, where we must deal with a $4 \times 4$ non-symmetric mass matrix (or a $5 \times 5$, if we consider the contribution of the charged right-handed

higgsino fields $\tilde{\Delta}_{R}$ and $\tilde{\delta}_{R}$ ), it is suitable to dispose of a method that allows us to disentangle the parameters in a systematic manner.

For a complete description of the chargino observables, in addition to the projectors matrices, it is also necessary to compute the so-called pseudoprojector matrices and CP eigenphases. In the following we implement a method based on the knowledge of the general structure of the diagonalizing matrices $U$ and $V$ to obtain these quantities and demonstrate some of their properties. 
Since two unitary matrices are needed to diagonalize $M$, we must define two classes of projector matrices $P^{U}$ and $P^{V}$ associated to the diagonalizing matrices $U$ and $V$, respectively, and a class of pseudoprojector matrix $\bar{P}$. They are defined as[17]:

$$
\begin{gathered}
P_{j}^{U^{*}}=\left(P_{j}^{U^{*}}\right)^{\dagger}=U^{*} E_{j} U^{T} \\
P_{j}^{V}=\left(P_{j}^{V}\right)^{\dagger}=V E_{j} V^{-1} \\
\bar{P}_{j}=U^{*} E_{j} V^{-1}
\end{gathered}
$$

so that

$$
\begin{gathered}
P_{j \alpha \beta}^{U^{*}}=U_{\alpha j}^{*} U_{\beta j}, \\
P_{j \alpha \beta}^{V}=V_{\alpha j} V_{\beta j}^{*}, \\
\bar{P}_{j \alpha \beta}=U_{\alpha j}^{*} V_{\beta j}^{*} .
\end{gathered}
$$

These projectors and pseudoprojectors satisfy the relations

$$
\begin{gathered}
P_{i}^{U^{*}} P_{j}^{U^{*}}=P_{j}^{U^{*}} \delta_{i j}, \quad \operatorname{Tr} P_{j}^{U^{*}}=1, \quad \sum_{j=1}^{4} P_{j}^{U^{*}}=1, \\
P_{i}^{V} P_{j}^{V}=P_{j}^{V} \delta_{i j}, \quad \operatorname{Tr} P_{j}^{V}=1, \quad \sum_{j=1}^{4} P_{j}^{V}=1, \\
\bar{P}_{j} \bar{P}_{j}^{\dagger}=P_{j}^{U^{*}}, \quad \bar{P}_{j}^{\dagger} \bar{P}_{j}=P_{j}^{V}, \\
P_{j}^{U^{*}} \bar{P}_{j}=\bar{P}_{j} P_{j}^{V}=\bar{P}_{j} .
\end{gathered}
$$

Note that from Eqs. (3.25), (5.39) and (5.40) it is possible to write

$$
M M^{\dagger}=\sum_{j=1}^{4} m_{\tilde{\chi}_{j}^{ \pm}}^{2} P_{j}^{U^{*}}
$$

and

$$
M^{\dagger} M=\sum_{j=1}^{4} m_{\tilde{\chi}_{j}^{ \pm}}^{2} P_{j}^{V} .
$$

Also, from Eqs. (3.24) and (5.41), we can write

$$
M=\sum_{j=1}^{4} m_{\tilde{\chi}_{j}^{ \pm}} U^{*} E_{j} V^{-1}=\sum_{j=1}^{4} m_{\tilde{\chi}_{j}^{ \pm}} \bar{P}_{j} .
$$




\subsection{Reduced projectors}

The projector and pseudoprojector matrices can be expressed in terms of the most fundamental ones, the so-called reduced projectors [13]. Indeed, by inserting (4.30) into (5.43), we get

$$
P_{j \alpha \beta}^{V}=\frac{p_{j \alpha}^{*} p_{j \beta}}{\left|p_{j 1}\right|^{2}+\left|p_{j 2}\right|^{2}+\left|p_{j 3}\right|^{2}+\left|p_{j 4}\right|^{2}}
$$

where we have define the type- $V$ reduced projectors [11]

$$
p_{j \alpha} \equiv \frac{\Delta_{\alpha j}^{*}(H)}{\Delta_{1 j}^{*}(H)} .
$$

Thus, from Eq. (5.52) we deduce

$$
P_{j 11}^{V}=\frac{1}{\left|p_{j 1}\right|^{2}+\left|p_{j 2}\right|^{2}+\left|p_{j 3}\right|^{2}+\left|p_{j 4}\right|^{2}}
$$

Inserting this last result into (5.52), we get

$$
P_{j \alpha \beta}^{V}=P_{j 11}^{V} p_{j \alpha}^{*} p_{j \beta}
$$

In the same way, substituting (4.33) into (5.42), we get

$$
P_{j \alpha \beta}^{U^{*}}=\frac{\tilde{p}_{j \alpha}^{*} \tilde{p}_{j \beta}}{\left|\tilde{p}_{j 1}\right|^{2}+\left|\tilde{p}_{j 2}\right|^{2}+\left|\tilde{p}_{j 3}\right|^{2}+\left|\tilde{p}_{j 4}\right|^{2}},
$$

where we have define the type- $U^{*}$ reduced projectors

$$
\tilde{p}_{j \alpha} \equiv \frac{\tilde{\Delta}_{\alpha j}^{*}(\tilde{H})}{\tilde{\Delta}_{1 j}^{*}(\tilde{H})} .
$$

Thus, from Eq. (5.56) we deduce

$$
P_{j 11}^{U^{*}}=\frac{1}{\left|\tilde{p}_{j 1}\right|^{2}+\left|\tilde{p}_{j 2}\right|^{2}+\left|\tilde{p}_{j 3}\right|^{2}+\left|\tilde{p}_{j 4}\right|^{2}} .
$$

Inserting this last result into (5.56), we get

$$
P_{j \alpha \beta}^{U^{*}}=P_{j 11}^{U^{*}} \tilde{p}_{j \alpha}^{*} \tilde{p}_{j \beta}
$$

On the other hand, using Eqs. (5.53) and (5.54), we can express the entries of the diagonalizing matrix $V$ given in Eq. (4.30) in terms of the type- $V$ reduced projectors, that is

$$
V_{\alpha j}=\sqrt{\frac{P_{j 11}^{V}}{\eta_{j}}} p_{j \alpha}^{*},
$$

where $\eta_{j} \equiv e^{-2 i \theta_{j}}$ stands for the type- $V$ CP eigenphases. Similarly, using Eqs. (5.57) and (5.58), we can express the entries of the diagonalizing matrix $U^{*}$ given in Eq. (4.33) in terms of the type- $U^{*}$ reduced projectors, that is

$$
U_{\alpha j}^{*}=\sqrt{\frac{P_{j 11}^{U^{*}}}{\tilde{\eta}_{j}}} \tilde{p}_{j \alpha}^{*},
$$


where $\tilde{\eta}_{j} \equiv e^{2 i \tilde{\theta}_{j}}$ stands for the type- $U^{*} \mathrm{CP}$ eigenphases.

Note that the reduced projector are not independent. As $V$ and $U^{*}$ are unitary matrices, using Eqs. (5.60) and (5.61), we get the constraints

$$
\sum_{k=1}^{4} p_{i k} p_{j k}^{*}=0
$$

and

$$
\sum_{k=1}^{4} \tilde{p}_{i k} \tilde{p}_{j k}^{*}=0
$$

when $i, j=1, \ldots, 4, / i>j$. Eqs. (5.62) and (5.63) represents each one a system of six complex algebraic equations serving to reduce, in the most general case, up to twelve the number of real independent parameters on each set of reduced projectors.

Inserting Eqs. (5.60) and (5.61) into Eq. (5.44) we can express the pseudoprojector entries in terms of the reduced projectors and eigenphases $\zeta_{j}=\sqrt{\eta_{j} / \tilde{\eta}_{j}}$ :

$$
\bar{P}_{j \alpha \beta}=\zeta_{j} \sqrt{P_{j 11}^{U^{*}} P_{j 11}^{V}} \tilde{p}_{j \alpha}^{*} p_{j \beta} .
$$

Moreover, substituting Eq. (5.60) into Eq. (4.37), we obtain

$$
U_{\alpha j}^{*}=\frac{1}{m_{\tilde{\chi}_{j}^{ \pm}}} \sqrt{\frac{P_{j 11}^{V}}{\eta_{j}}} \sum_{k=1}^{4} M_{\alpha k} p_{j k}^{*},
$$

so from the equivalence between Eqs. (5.61) and (5.65), we deduce $(\alpha=1, \ldots, 4)$

$$
m_{\tilde{\chi}_{j}^{ \pm}} \zeta_{j} \sqrt{\frac{P_{j 11}^{U^{*}}}{P_{j 11}^{V}}} \tilde{p}_{j \alpha}^{*}=\sum_{\beta=1}^{4} M_{\alpha \beta} p_{j \beta}^{*} .
$$

On the other hand, following the same procedure, from the corresponding equivalence for the entries of the $V$ matrix, we get $(\alpha=1, \ldots, 4)$

$$
m_{\tilde{\chi}_{j}^{ \pm}} \zeta_{j} \sqrt{\frac{P_{j 11}^{V}}{P_{j 11}^{U^{*}}}} p_{j \alpha}=\sum_{\beta=1}^{4} M_{\beta \alpha} \tilde{p}_{j \beta} .
$$

Equations (5.66) and (5.67) constitute a generalization of the corresponding formulas deduced in the case of the neutralino $[11,13]$ which was based on a complex symmetric mass matrix. These equations represent, for fixed $j$, a system of eight complex algebraic equations serving to determine the fundamental parameters of the model in terms of the reduced projectors, the chargino physical masses $m_{\tilde{\chi}_{j}^{ \pm}}$and the eigenphases, or vice versa.

Note that, in Eqs. (5.60) and (5.61) as well as in Eqs. (5.66) and (5.67), without any loss of generality, we could choose the $U^{*}$-type eigenphases either $\tilde{\eta}_{j}=1, j=1, \ldots, 4$, such that $\zeta_{j}=\sqrt{\eta_{j}} ; \tilde{\eta}_{j}^{*}=\eta_{j}, j=$ $1,2, \ldots, 4$, such $\zeta_{j}=\eta_{j}$, or any other suitable choice allowing us to eliminate four superfluous parameters. 


\subsection{Explicit form of the reduced projectors}

In general, according to Eqs. (5.53) and (5.57), to obtain the explicit form of the reduced projectors of the $V$-type and $U^{*}$-type, in terms of the fundamental parameters of the theory, we only need to know the explicit form of quantities $\Delta_{\alpha j}^{*}$ and $\tilde{\Delta}_{\alpha j}^{*}$, respectively. For fixed $j=1,2,4$ the quantities of the $V$-type are given by

$$
\begin{aligned}
\Delta_{1 j}^{*}= & \left(|\mu|^{2}-m_{\tilde{\chi}_{j}^{ \pm}}^{2}\right)\left\{\left(|\mu|^{2}-m_{\tilde{\chi}_{j}^{ \pm}}^{2}\right)\left(M_{R}^{2}-m_{\tilde{\chi}_{j}^{ \pm}}^{2}\right)\right. \\
- & 2 \cos ^{2} \theta_{k}\left|\tilde{M}_{L}\right|^{2}\left(m_{\tilde{\chi}_{j}^{ \pm}}^{2}-M_{R}^{2}-2\left|\tilde{M}_{R}\right|^{2} \sin ^{2} \theta_{k}\right) \\
- & 2\left|\tilde{M}_{R}\right|^{2} \\
\times & {\left[m_{\tilde{\chi}_{j}^{ \pm}}^{2}-M_{R}|\mu| \cos \left(2 \tilde{\Phi}_{R}-\Phi_{\mu}\right) \sin \left(2 \theta_{k}\right)\right] } \\
+ & \left.\left|\tilde{M}_{R}\right|^{4} \sin ^{2}\left(2 \theta_{k}\right)\right\} \\
\Delta_{2 j}^{*} & =2\left|\tilde{M}_{L}\right|\left|\tilde{M}_{R}\right|\left(m_{\tilde{\chi}_{j}^{ \pm}}^{2}-|\mu|^{2}\right) \\
& \times\left\{\operatorname { s i n } \theta _ { k } \left[|\mu| M_{R} \cos \theta_{k} e^{i\left(\Phi_{u}-\tilde{\Phi}_{L}-\tilde{\Phi}_{R}\right)}\right.\right. \\
& +\sin \theta_{k} e^{i\left(\tilde{\Phi}_{R}-\tilde{\Phi}_{L}\right)} \\
& \left.\times\left[2\left(\left|\tilde{M}_{L}\right|^{2}+\left|\tilde{M}_{R}\right|^{2}\right) \cos ^{2} \theta_{k}-m_{\tilde{\chi}_{j}^{ \pm}}^{2}\right]\right] \\
& +\left|M_{L}\right| \cos \theta_{k}\left[|\mu| \sin \theta_{k} e^{-i\left(\Phi_{u}+\Phi_{L}-\tilde{\Phi}_{L}-\tilde{\Phi}_{R}\right)}\right. \\
& \left.\left.-M_{R} \cos \theta_{k} e^{-i\left(\Phi_{L}-\tilde{\Phi}_{L}+\tilde{\Phi}_{R}\right)}\right]\right\} \\
& \quad \Delta_{3 j}^{*}=0
\end{aligned}
$$

and

$$
\begin{aligned}
\Delta_{4 j}^{*} & =\sqrt{2}\left|\tilde{M}_{L}\right|\left(m_{\tilde{\chi}_{j}^{ \pm}}^{2}-|\mu|^{2}\right) \\
& \times\left\{\left|M_{L}\right| \cos \theta_{k} e^{i\left(\tilde{\Phi}_{L}-\Phi_{L}\right)}\right. \\
& \times\left[M_{R}^{2}-m_{\tilde{\chi}_{j}^{ \pm}}^{2}+2\left|\tilde{M}_{R}\right|^{2} \sin ^{2} \theta_{k}\right]+\sin \theta_{k} \\
& \times\left[|\mu|\left(m_{\tilde{\chi}_{j}^{ \pm}}^{2}-|\mu|^{2}\right) e^{i\left(\Phi_{\mu}-\tilde{\Phi}_{L}\right)}\right. \\
& \left.\left.-M_{R}\left|\tilde{M}_{R}\right|^{2} \sin \left(2 \theta_{k}\right) e^{i\left(2 \tilde{\Phi}_{R}-\tilde{\Phi}_{L}\right)}\right]\right\},
\end{aligned}
$$

and the ones of the $U^{*}$-type are given by

$$
\begin{aligned}
\tilde{\Delta}_{1 j}^{*} & =\left(|\mu|^{2}-m_{\tilde{\chi}_{j}^{ \pm}}^{2}\right)\left\{\left(|\mu|^{2}-m_{\tilde{\chi}_{j}^{ \pm}}^{2}\right)\left(M_{R}^{2}-m_{\tilde{\chi}_{j}^{ \pm}}^{2}\right)\right. \\
& -2 \sin ^{2} \theta_{k}\left|\tilde{M}_{L}\right|^{2}\left(m_{\tilde{\chi}_{j}^{ \pm}}^{2}-M_{R}^{2}-2\left|\tilde{M}_{R}\right|^{2} \cos ^{2} \theta_{k}\right) \\
& -2\left|\tilde{M}_{R}\right|^{2} \\
& \times\left[m_{\tilde{\chi}_{j}^{ \pm}}^{2}-M_{R}|\mu| \cos \left(2 \tilde{\Phi}_{R}-\Phi_{\mu}\right) \sin \left(2 \theta_{k}\right)\right] \\
& \left.+\left|\tilde{M}_{R}\right|^{4} \sin ^{2}\left(2 \theta_{k}\right)\right\}
\end{aligned}
$$




$$
\begin{aligned}
\tilde{\Delta}_{2 j}^{*} & =2\left|\tilde{M}_{L}\right|\left|\tilde{M}_{R}\right|\left(m_{\tilde{\chi}_{j}^{ \pm}}^{2}-|\mu|^{2}\right) \\
& \times\left\{\operatorname { c o s } \theta _ { k } \left[|\mu| M_{R} \sin \theta_{k} e^{-i\left(\Phi_{u}-\tilde{\Phi}_{L}-\tilde{\Phi}_{R}\right)}\right.\right. \\
& +\cos \theta_{k} e^{i\left(\tilde{\Phi}_{L}-\tilde{\Phi}_{R}\right)} \\
& \left.\times\left[2\left(\left|\tilde{M}_{L}\right|^{2}+\left|\tilde{M}_{R}\right|^{2}\right) \sin ^{2} \theta_{k}-m_{\tilde{\chi}_{j}^{ \pm}}^{2}\right]\right] \\
& +\left|M_{L}\right| \sin \theta_{k}\left[|\mu| \cos \theta_{k} e^{i\left(\Phi_{u}+\Phi_{L}-\tilde{\Phi}_{L}-\tilde{\Phi}_{R}\right)}\right. \\
& \left.\left.-M_{R} \sin \theta_{k} e^{i\left(\Phi_{L}-\tilde{\Phi}_{L}+\tilde{\Phi}_{R}\right)}\right]\right\}, \\
\tilde{\Delta}_{3 j}^{*} & =\sqrt{2}\left|\tilde{M}_{L}\right|\left(m_{\tilde{\chi}_{j}^{ \pm}}^{2}-|\mu|^{2}\right) \\
& \times\left\{\left|M_{L}\right| \sin \theta_{k} e^{-i\left(\tilde{\Phi}_{L}-\Phi_{L}\right)}\right. \\
\times & {\left[M_{R}^{2}-m_{\tilde{\chi}_{j}^{ \pm}}^{2}+2\left|\tilde{M}_{R}\right|^{2} \cos ^{2} \theta_{k}\right]+\cos \theta_{k} } \\
& \times\left[|\mu|\left(m_{\tilde{\chi}_{j}^{ \pm}}^{2}-|\mu|^{2}\right) e^{-i\left(\Phi_{\mu}-\tilde{\Phi}_{L}\right)}\right. \\
& \left.\left.-M_{R}\left|\tilde{M}_{R}\right|^{2} \sin \left(2 \theta_{k}\right) e^{-i\left(2 \tilde{\Phi}_{R}-\tilde{\Phi}_{L}\right)}\right]\right\},
\end{aligned}
$$

and

$$
\tilde{\Delta}_{4 j}^{*}=0
$$

We note that the quantities $\tilde{\Delta}_{i j}^{*}, i=1,2$ can be obtained from $\Delta_{i j}^{*}, i=1,2$ by interchanging $\sin \theta_{k} \leftrightarrow \cos \theta_{k}$ and then taking the complex conjugate. The quantity $\tilde{\Delta}_{3 j}$, can be obtained from $\Delta_{4 j}^{*}$ in the same way.

As the delta factors becomes singular when $j=3$, because the particular values taken by the entries of the $H$ and $\tilde{H}$ matrices and the fact that $m_{\tilde{\chi}_{3}^{ \pm}}=|\mu|$, see Appendix A, the $p_{j \alpha}$ 's and $\tilde{p}_{j, \alpha}$ 's, when $j=3$, must be computed separately taking into account the unitary character of the $V$ and $U^{*}$ matrices. Using Eqs.(5.60) and (5.61), we get $p_{33}=\tilde{p}_{34}=1, p_{3 \alpha}=p_{\alpha 3}=\tilde{p}_{\alpha 4}=0, \alpha=1,2,4$, and $\tilde{p}_{3 \alpha}=0, \alpha=1,2,3$.

The quantities given in Eqs. (5.68-5.75) allow us to express, through the reduced projectors, all the essential quantities of the model in terms of the original parameters.

\subsection{Fundamental parameters in terms of the reduced projectors}

Equations (5.66) and (5.67) allows us to perform a change of parametrization from the original fundamental parameters to the reduced projectors and eigenphases. This change of parametrization is suitable when we essay to fix the value of the fundamental parameters by measuring physical observables which explicitly involves the entries of the diagonalizing $U^{*}$ and $V$ matrices such as the cross-section-type observables associated to the chargino pair production through unpolarized or polarized $e^{+} e^{-}$annihilation processes, for instance, total cross sections, asymmetries, T-odd asymmetries and polarization vectors. Indeed, in such a case, the relevant quantities depends directly on the entries of the diagonalizing $V$ and $U^{*}$ matrices, i.e., they depends in a relative simple forme on the reduced projectors and eigenphases. Thus, experimental measurements of this class of observables provide us of independent constraints serving to determinate, in principle, these eigenphases and reduced projectors, i.e., inserting these results into the relations described immediately below allows us to reconstruct all the fundamental L-R SUSY parameters. 
Recalling that $M_{R}$ is a real parameter, from Eqs. (5.66) and (5.67), we get $(\mathrm{j}=1,2,4)$

$$
\begin{aligned}
& M_{L}=m_{\tilde{\chi}_{j}^{ \pm}} \zeta_{j} \frac{\sqrt{\frac{P_{j 11}^{U^{*}}}{P_{j 11}^{V}}} \tilde{p}_{j 3} \tan \theta_{k}-\sqrt{\frac{P_{j 11}^{V}}{P_{j 11}^{U^{*}}}} p_{j 4}^{*}}{\tilde{p}_{j 3} \tan \theta_{k}-p_{j 4}^{*}} \\
& \tilde{M}_{L}=-\frac{m_{\tilde{\chi}_{j}^{ \pm}} \zeta_{j}}{\sqrt{2}} \frac{\sqrt{\frac{P_{j 11}^{U^{*}}}{P_{j 11}^{V}}}-\sqrt{\frac{P_{j 11}^{V}}{P_{j 11}^{U^{*}}}}}{\tilde{p}_{j 3} \sin \theta_{k}-p_{j 4}^{*} \cos \theta_{k}} \\
& \tilde{M}_{R}=\frac{m_{\tilde{\chi}_{j}^{ \pm}} \zeta_{j}}{\sqrt{2}} \frac{\sqrt{\frac{P_{j 11}^{U^{*}}}{P_{j 11}^{V}}}\left|\tilde{p}_{j 2}\right|^{2}-\sqrt{\frac{P_{j 11}^{V}}{P_{j 11}^{U^{*}}}}\left|p_{j 2}\right|^{2}}{p_{j 4}^{*} \tilde{p}_{j 2} \cos \theta_{k}-p_{j 2}^{*} \tilde{p}_{j 3} \sin \theta_{k}}, \\
& \mu=\frac{m_{\tilde{\chi}_{j}^{ \pm}} \zeta_{j}}{\left(p_{j 4}^{*}-\tilde{p}_{j 3} \tan \theta_{k}\right)\left(\tilde{p}_{j 3} p_{j 2}^{*} \tan \theta_{k}-\tilde{p}_{j 2} p_{j 4}^{*}\right)} \\
& \times\left\{\sqrt{\frac{P_{j 11}^{U^{*}}}{P_{j 11}^{V}}}\left[\tilde{p}_{j 2} \tilde{p}_{j 3}^{*} p_{j 4}^{*}-\left(\left(p_{j 2}^{*}-\tilde{p}_{j 2}\right)\left|\tilde{p}_{j 2}\right|^{2}+p_{j 2}^{*}\left|\tilde{p}_{j 3}\right|^{2}\right) \tan \theta_{k}\right]\right.
\end{aligned}
$$

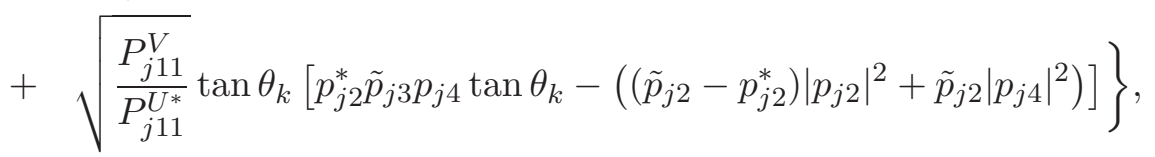

$$
\begin{aligned}
& M_{R}=\operatorname{sign}\left(\operatorname{Re} Z_{j}\right) m_{\tilde{\chi}_{j}^{ \pm}}\left|Z_{j}\right|, \quad \text { if } \operatorname{Re} Z_{j} \neq 0, \quad M_{R}=\mp m_{\tilde{\chi}_{j}^{ \pm}} \operatorname{Im} Z_{j}, \quad \text { if } \operatorname{Re} Z_{j}=0,
\end{aligned}
$$

and

$$
\zeta_{j}=\operatorname{sign}\left(\operatorname{Re} Z_{j}\right) \frac{Z_{j}^{*}}{\left|Z_{j}\right|}, \quad \text { if } \operatorname{Re} Z_{j} \neq 0, \quad \zeta_{j}= \pm i, \quad \text { if } \operatorname{Re} Z_{j}=0
$$

where

$$
Z_{j}=\frac{\sqrt{\frac{P_{j 1}^{U^{*}}}{P_{j 11}^{V}}} \tilde{p}_{j 2}^{*} \tilde{p}_{j 3} \tan \theta_{k}-\sqrt{\frac{P_{j 11}^{V}}{P_{j 11}^{U *}}} p_{j 2} p_{j 4}^{*}}{p_{j 2}^{*} \tilde{p}_{j 3} \tan \theta_{k}-\tilde{p}_{j 2} p_{j 4}^{*}} .
$$

When $j=3$, we only have

$$
\zeta_{3}=-\frac{\mu}{|\mu|} .
$$

Note that in the CP-conserving case $\operatorname{Im} Z_{j}=0$, and $\Phi_{\mu}=0, \pi$ i.e., from Eqs. (5.81) and (5.83), we get $\zeta_{j}= \pm 1, j=1, \ldots, 4$. Thus, in the CP-conserving case, there is not an analogous to the equation (5.81) that allows us to express $\tan \theta_{k}$ in terms of the eigenphases and reduced projectors, so in order to express the fundamental parameters in terms of them using Eqs.(5.76-5.80), we must known $\tan \theta_{k}$ from some other means. In the $\mathrm{CP}$-conserving case, the role of the eigenphases is to remedy the sign ambiguity of the physical chargino masses represented by the eigenvalues of an Hermitian matrix $H$ or $\tilde{H}$, which can be either positive or negative $[12,13]$. In addition to this, in the $\mathrm{CP}$-violating case, the eigenphases contain information on the complex phases introduced in the chargino mass matrix .

On the other hand, we note that using Eqs. (5.55), (5.59) and (5.64), we can express all the fundamental parameters completely in terms of the $U^{*}$-, $V$-type projectors, $P_{j}^{U^{*}}, P_{j}^{V}$ and pseudoprojectors, $\bar{P}_{j}$. 


\subsection{Equivalent formulas for the reduced projectors}

Using the Jarlskog's formulas [17], we can show that the projectors of the type $V$ and $U^{*}$ can be written in the form $[11,12,13](j=1,2,4)$

$$
\begin{aligned}
P_{j}^{V} & =\frac{\tilde{P}_{j}^{V}}{\tilde{\Delta}_{j}}, \\
P_{j}^{U^{*}} & =\frac{\tilde{P}_{j}^{U^{*}}}{\tilde{\Delta}_{j}},
\end{aligned}
$$

respectively, where

$$
\tilde{\Delta}_{j}=-3 m_{\tilde{\chi}_{j}^{ \pm}}^{8}+2 a m_{\tilde{\chi}_{j}^{ \pm}}^{6}-b m_{\tilde{\chi}_{j}^{ \pm}}^{4}+d .
$$

Thus entries of these projector matrices can be written in the form [11]

$$
\begin{aligned}
\tilde{P}_{j \alpha \beta}^{V} & =-m_{\tilde{\chi}_{j}^{ \pm}}^{6} H_{\alpha \beta}+m_{\tilde{\chi}_{j}^{ \pm}}^{4}\left(a H_{\alpha \beta}-H_{\alpha \beta}^{2}\right) \\
& +m_{\tilde{\chi}_{j}^{ \pm}}^{2}\left(a H_{\alpha \beta}^{2}-b H_{\alpha \beta}-H_{\alpha \beta}^{3}\right) \\
& +d \delta_{\alpha \beta}
\end{aligned}
$$

and

$$
\begin{aligned}
\tilde{P}_{j \alpha \beta}^{U^{*}} & =-m_{\tilde{\chi}_{j}^{ \pm}}^{6} \tilde{H}_{\alpha \beta}+m_{\tilde{\chi}_{j}^{ \pm}}^{4}\left(a \tilde{H}_{\alpha \beta}-\tilde{H}_{\alpha \beta}^{2}\right) \\
& +m_{\tilde{\chi}_{j}^{ \pm}}^{2}\left(a \tilde{H}_{\alpha \beta}^{2}-b \tilde{H}_{\alpha \beta}-\tilde{H}_{\alpha \beta}^{3}\right) \\
& +d \delta_{\alpha \beta},
\end{aligned}
$$

Now, combining Eqs. (5.55) and (5.84) and Eqs. (5.59) and (5.85), we deduce

$$
p_{j \alpha}=\frac{P_{j 1 \alpha}^{V}}{P_{j 11}^{V}}=\frac{\tilde{P}_{j 1 \alpha}^{V}}{\tilde{P}_{j 11}^{V}}
$$

and

$$
\tilde{p}_{j \alpha}=\frac{P_{j 1 \alpha}^{U^{*}}}{P_{j 11}^{U^{*}}}=\frac{\tilde{P}_{j 1 \alpha}^{U^{*}}}{\tilde{P}_{j 11}^{U^{*}}} .
$$

Equations (5.53) and (5.89) are equivalent expressions. Thus, combining these equations and comparing the expressions (5.69-5.71) with the corresponding $\tilde{P}_{j 1 \beta}, \beta=2,3,4$, computed from Eq. (5.87), we can show that

$$
\tilde{P}_{j 1 \alpha}^{V}=m_{\tilde{\chi}_{j}^{ \pm}}^{2} \Delta_{\alpha j}^{*}, \quad \forall \alpha=1, \ldots, 4,
$$

with 


$$
\begin{aligned}
\tilde{P}_{j 11}^{V} & =-\frac{1}{2}\left(m_{\tilde{\chi}_{j}^{ \pm}}^{2}-|\mu|^{2}\right)\left\{4 | \tilde { M } _ { L } | ^ { 2 } \operatorname { s i n } ^ { 2 } \theta _ { k } \left[m_{\tilde{\chi}_{j}^{ \pm}}^{2}\right.\right. \\
& \times\left(m_{\tilde{\chi}_{j}^{ \pm}}^{2}-M_{R}^{2}-2\left|\tilde{M}_{R}\right|^{2} \cos ^{2} \theta_{k}\right)-2\left|\tilde{M}_{L}\right|^{2} \\
& \left.\times\left(m_{\tilde{\chi}_{j}^{ \pm}}^{2}-M_{R}^{2}\right) \cos ^{2} \theta_{k}\right]+4\left|M_{L}\right|\left|\tilde{M}_{L}\right|^{2} \sin \left(2 \theta_{k}\right) \\
& \times\left[M_{R}\left|\tilde{M}_{R}\right|^{2} \cos \left(\Phi_{L}-2 \tilde{\Phi}_{L}+2 \tilde{\Phi}_{R}\right) \sin \left(2 \theta_{k}\right)\right. \\
& \left.-|\mu|\left(m_{\tilde{\chi}_{j}^{ \pm}}^{2}-M_{R}^{2}\right) \cos \left(\Phi_{L}-2 \tilde{\Phi}_{L}+\Phi_{\mu}\right)\right] \\
& +2\left|M_{L}\right|^{2}\left[\left(m_{\tilde{\chi}_{j}^{ \pm}}^{2}-M_{R}^{2}\right)\left(m_{\tilde{\chi}_{j}^{ \pm}}^{2}-|\mu|^{2}\right)-2\left|\tilde{M}_{R}\right|^{2}\right. \\
& \times\left(m_{\tilde{\chi}_{j}^{ \pm}}^{2}-|\mu| M_{R} \cos \left(2 \tilde{\Phi}_{R}-\Phi_{\mu}\right) \sin \left(2 \theta_{k}\right)\right. \\
& \left.\left.+\left|\tilde{M}_{R}\right|^{4} \sin ^{2}\left(2 \theta_{k}\right)\right]\right\} .
\end{aligned}
$$

In the same way, combining Eqs. (5.57) and (5.90) and comparing the expressions (5.73-5.75), with the corresponding $\tilde{P}_{j 1 \beta}^{U^{*}}, \beta=2,3,4$, computed from Eq. (5.88), we can show that

$$
\tilde{P}_{j 1 \alpha}^{U^{*}}=m_{\tilde{\chi}_{j}^{ \pm}}^{2} \tilde{\Delta}_{\alpha j}^{*}, \quad \forall \alpha=1, \ldots, 4,
$$

with

$$
\begin{aligned}
\tilde{P}_{j 11}^{U^{*}} & =-\frac{1}{2}\left(m_{\tilde{\chi}_{j}^{ \pm}}^{2}-|\mu|^{2}\right)\left\{4 | \tilde { M } _ { L } | ^ { 2 } \operatorname { c o s } ^ { 2 } \theta _ { k } \left[m_{\tilde{\chi}_{j}^{ \pm}}^{2}\right.\right. \\
& \times\left(m_{\tilde{\chi}_{j}^{ \pm}}^{2}-M_{R}^{2}-2\left|\tilde{M}_{R}\right|^{2} \sin ^{2} \theta_{k}\right)-2\left|\tilde{M}_{L}\right|^{2} \\
& \left.\times\left(m_{\tilde{\chi}_{j}^{ \pm}}^{2}-M_{R}^{2}\right) \sin ^{2} \theta_{k}\right]+4\left|M_{L}\right|\left|\tilde{M}_{L}\right|^{2} \sin \left(2 \theta_{k}\right) \\
& \times\left[M_{R}\left|\tilde{M}_{R}\right|^{2} \cos \left(\Phi_{L}-2 \tilde{\Phi}_{L}+2 \tilde{\Phi}_{R}\right) \sin \left(2 \theta_{k}\right)\right. \\
& \left.-|\mu|\left(m_{\tilde{\chi}_{j}^{ \pm}}^{2}-M_{R}^{2}\right) \cos \left(\Phi_{L}-2 \tilde{\Phi}_{L}+\Phi_{\mu}\right)\right] \\
& +2\left|M_{L}\right|^{2}\left[\left(m_{\tilde{\chi}_{j}^{ \pm}}^{2}-M_{R}^{2}\right)\left(m_{\tilde{\chi}_{j}^{ \pm}}^{2}-|\mu|^{2}\right)-2\left|\tilde{M}_{R}\right|^{2}\right. \\
& \times\left(m_{\tilde{\chi}_{j}^{ \pm}}^{2}-|\mu| M_{R} \cos \left(2 \tilde{\Phi}_{R}-\Phi_{\mu}\right) \sin \left(2 \theta_{k}\right)\right. \\
& \left.\left.+\left|\tilde{M}_{R}\right|^{4} \sin ^{2}\left(2 \theta_{k}\right)\right]\right\} .
\end{aligned}
$$

Note that Eq. (5.91), for $\alpha=2,3,4$ constitute an identity whereas for $\alpha=1$, it constitutes an equivalence. The same argument is valid for Eq. (5.93). Also, note that $\tilde{P}_{j 11}^{U^{*}}$ can be obtained from $\tilde{P}_{j 11}^{V}$ by interchanging $\sin \theta_{k} \leftrightarrow \cos \theta_{k}$.

\section{Disentangling L-R SUSY parameters}

In this section we use the results of previous section to disentangle $M_{L}$ from the rest of the fundamental parameters. We present two equivalent ways to express the norm of $M_{L}$, first in terms of the eigenphases and second in terms of the phase angle $\Phi_{L}$. Also, we propose an alternative change of parametrization to disentangle $M_{R}$ from the rest of parameters. 


\subsection{Expressing $M_{L}$ in terms of the eigenphases}

From Eq. (5.66) for $\alpha=1$, considering that $\tilde{p}_{j 1}=1, j=1,2,4$, and using (2.12), we get

$$
m_{\tilde{\chi}_{j}^{ \pm}} \zeta_{j} \sqrt{\frac{\tilde{P}_{j 11}^{U^{*}}}{\tilde{P}_{j 11}^{V}}}=M_{L}+\sqrt{2} \tilde{M}_{L} \cos \theta_{k} p_{j 4}^{*} .
$$

Inserting Eqs. (5.84) and (5.85) into Eq. (6.95) and taking into account the equivalences given in Eqs. (5.91) and (5.93) as well as the fact that $p_{j 4}^{*}=\Delta_{4 j} / \Delta_{1 j}$, we obtain

$$
m_{\tilde{\chi}_{j}^{ \pm}} \zeta_{j} \sqrt{\frac{\tilde{\Delta}_{1 j}}{\Delta_{1 j}}}=M_{L}+\sqrt{2} \tilde{M}_{L} \cos \theta_{k} \frac{\Delta_{4 j}}{\Delta_{1 j}} .
$$

Now, substituting into (6.96) the values of $\Delta_{1 j}$ and $\Delta_{4 j}$ given in Eqs. (5.70) and (5.71), respectively, and solving a linear algebraic equation for $M_{L}$, we get

$$
M_{L}=\tilde{A}_{j} \zeta_{j}+\tilde{B}_{j}, \quad j=1,2,4
$$

where

$$
\tilde{A}_{j}=-\frac{\sqrt{\tilde{\Delta}_{1 j} \Delta_{1 j}}}{\left(m_{\tilde{\chi}_{j}^{ \pm}}^{2}-|\mu|^{2}\right)} \frac{m_{\tilde{\chi}_{j}^{ \pm}}}{\tilde{\mathcal{D}}_{j}}
$$

and

$$
\begin{aligned}
\tilde{B}_{j} & =\frac{\tilde{M}_{L}^{2} \sin \left(2 \theta_{k}\right)}{\tilde{\mathcal{D}}_{j}}\left[\left(m_{\tilde{\chi}_{j}^{ \pm}}^{2}-M_{R}^{2}\right)|\mu| e^{-i \Phi_{\mu}}\right. \\
& \left.-M_{R}\left|\tilde{M}_{R}\right|^{2} e^{-2 i \tilde{\Phi}_{R}} \sin \left(2 \theta_{k}\right)\right]
\end{aligned}
$$

where

$$
\begin{aligned}
\tilde{\mathcal{D}}_{j} & =\left|\tilde{M}_{R}\right|^{4} \sin ^{2}\left(2 \theta_{k}\right)+\left(m_{\tilde{\chi}_{j}^{ \pm}}^{2}-M_{R}^{2}\right)\left(m_{\tilde{\chi}_{j}^{ \pm}}^{2}-|\mu|^{2}\right) \\
& -2\left|\tilde{M}_{R}\right|^{2} \\
& \times\left[m_{\tilde{\chi}_{j}^{ \pm}}^{2}-|\mu| M_{R} \cos \left(2 \tilde{\Phi}_{R}-\Phi_{\mu}\right) \sin \left(2 \theta_{k}\right)\right] .
\end{aligned}
$$

Equation (6.97) allows us to determinate the behaviour of $\left|M_{L}\right|$ and $\Phi_{L}$ in terms of the eigenphases $\zeta_{j}$ and the physical masses $m_{\tilde{\chi}_{j}^{ \pm}}$, when the rest of fundamental parameters are known. The method used to obtain it is direct and is based essentially on the fact that $\Delta_{4 j}$ is a linear function of $M_{L}$ and both $\Delta_{1 j}$ and $\tilde{\Delta}_{1 j}$ are independent of $\left|M_{L}\right|$ and $\Phi_{L}$.

Note that the same result for $M_{L}$ can be obtained if first we solve the system of equations (5.665.67) for the reduced projectors $p_{j \alpha}, \tilde{p}_{j, \alpha}, \alpha=2,3,4$, without introducing any explicit dependence on the parameters $\left|M_{L}\right|, \Phi_{L}$, and then we insert these results into either Eq. (5.66) or (5.67) when $\alpha=1$. From the results of previous sections and using Eqs. (5.66) and (5.67), we get $(\mathrm{j}=1,2,4)$

$$
\begin{aligned}
p_{j 2} & =\frac{2}{\tilde{\mathcal{D}}_{j}}\left\{\tilde{M}_{L}^{*} \tilde{M}_{R}^{*} \sin \theta_{k}\left[e^{2 i \tilde{\Phi}_{R}} \sin \theta_{k}\left(m_{\tilde{\chi}_{j}^{ \pm}}^{2}-2\left|\tilde{M}_{R}\right|^{2} \cos ^{2} \theta_{k}\right)-\mu M_{R} \cos \theta_{k}\right]\right. \\
& \left.+\tilde{M}_{L} \tilde{M}_{R} \cos \theta_{k}\left[e^{-2 i \tilde{\Phi}_{R}} M_{R} \cos \theta_{k}-\mu^{*} \sin \theta_{k}\right] m_{\tilde{\chi}_{j}^{ \pm}} \zeta_{j}^{*} \sqrt{\frac{\tilde{\Delta}_{1 j}}{\Delta_{1 j}}}\right\},
\end{aligned}
$$




$$
\begin{aligned}
p_{j 4} & =\frac{\sqrt{2}}{\tilde{\mathcal{D}}_{j}}\left\{\tilde{M}_{L}^{*} \sin \theta_{k}\left[\mu\left(M_{R}^{2}-m_{\tilde{\chi}_{j}^{ \pm}}^{2}\right)+M_{R} \tilde{M}_{R}^{2} \sin \left(2 \theta_{k}\right)\right]\right. \\
& \left.+\tilde{M}_{L} \cos \theta_{k}\left[m_{\tilde{\chi}_{j}^{ \pm}}^{2}-M_{R}^{2}-2\left|\tilde{M}_{L}\right|^{2} \sin ^{2} \theta_{k}\right] m_{\tilde{\chi}_{j}^{ \pm}} \zeta_{j}^{*} \sqrt{\frac{\tilde{\Delta}_{1 j}}{\Delta_{1 j}}}\right\}
\end{aligned}
$$

and $\tilde{p}_{j 2}$ and $\tilde{p}_{j 3}$ are obtained from $p_{j 2}^{*}$ and $p_{j 4}^{*}$ by interchanging $\sin \theta_{k} \leftrightarrow \cos \theta_{k}$, respectively.

\subsection{Expressing $\left|M_{L}\right|$ in terms of $\Phi_{L}$}

When $\alpha=1$, either Eq. (5.91) combined with Eqs. (5.68) and (5.92) or Eq. (5.93) combined with Eqs. (5.72) and (5.94), allow us to express the norm of $M_{L}$ in terms of the physical masses $m_{\tilde{\chi}_{j}^{ \pm}}, \Phi_{L}$ and the rest of the fundamental parameters . In both cases we obtain identical results. Thus, for instance, inserting (5.68) and (5.92) into (5.91) (with $\alpha=1$ ) we get

$$
\tilde{\mathcal{D}}_{j}\left|M_{L}\right|^{2}+\tilde{\mathcal{B}}_{j}\left|M_{L}\right|+\tilde{\mathcal{C}}_{j}=0, \quad j=1,2,4,
$$

where

$$
\begin{aligned}
\tilde{\mathcal{B}}_{j} & =2\left|\tilde{M}_{L}\right|^{2} \sin \left(2 \theta_{k}\right) \\
& \times\left[M_{R}\left|\tilde{M}_{R}\right|^{2} \cos \left(\Phi_{L}-2 \tilde{\Phi}_{L}+2 \tilde{\Phi}_{R}\right) \sin \left(2 \theta_{k}\right)\right. \\
& \left.-|\mu|\left(m_{\tilde{\chi}_{j}^{ \pm}}^{2}-M_{R}^{2}\right) \cos \left(\Phi_{L}-2 \tilde{\Phi}_{L}+\Phi_{\mu}\right)\right], \\
\tilde{\mathcal{C}}_{j} & =-\left|\tilde{M}_{L}\right|^{4}\left(m_{\tilde{\chi}_{j}^{ \pm}}^{2}-M_{R}^{2}\right) \sin ^{2}\left(2 \theta_{k}\right)-m_{\tilde{\chi}_{j}^{ \pm}}^{2}\left[\tilde{\mathcal{D}}_{j}\right. \\
& \left.-2\left|\tilde{M}_{L}\right|^{2}\left[m_{\tilde{\chi}_{j}^{ \pm}}^{2}-M_{R}^{2}-\left|\tilde{M}_{R}\right|^{2} \sin ^{2}\left(2 \theta_{k}\right)\right]\right]
\end{aligned}
$$

and $\tilde{\mathcal{D}}_{j}$ is given by Eq. (6.100). Thus, solving a quadratic algebraic equation for $\left|M_{L}\right|$, we get

$$
\left|M_{L}\right|=\frac{-\tilde{\mathcal{B}}_{j} \pm \sqrt{\tilde{\mathcal{B}}_{j}^{2}-4 \tilde{\mathcal{D}}_{j} \tilde{\mathcal{C}}_{j}}}{2 \tilde{\mathcal{D}}_{j}}, \quad j=1,2,4
$$

From Eq. (6.106) we deduce the constraints $\tilde{\mathcal{B}}_{j}^{2}-4 \tilde{\mathcal{D}}_{j} \tilde{\mathcal{C}}_{j} \geq 0$ and $\tilde{\mathcal{B}}_{j} / \tilde{\mathcal{D}}_{j}<0$.

Equation (6.97) for $\left|M_{L}\right|$ is equivalent to Eq. (6.106). For instance, in the CP-conserving case, when all the mixing phases except $\Phi_{L}$ are equal to zero, the choice of the eigenphase values $\xi_{j}= \pm 1$ in Eq. (6.97) correspond to the choice of the values $\Phi_{L}= \pm \pi$, in Eq. (6.106), respectively. Thus, in the CP-violating case, when all the fundamental parameters except $M_{L}, \Phi_{L}$ and $\tilde{\Phi}_{L}$ are known, Eq. (6.106) allows the mixing angle $\Phi_{L}-2 \tilde{\Phi}_{L}$ to play the role of the eigenphases.

\subsection{Disentangling $M_{R}$}

The explicit form of $M_{R}$ in terms of some redefined eigenphases $\zeta_{j}^{(2)}$ and the rest of fundamental parameters can be deduced from Eqs. (6.97-6.100) by interchanging $M_{L}$ with $M_{R}$ and $\tilde{M}_{L}$ with $\tilde{M}_{R}$. This last affirmation is based on the fact that $M_{L}$ and $M_{R}$ play a similar role in Eqs. (A.5-A.6) provided $\tilde{M}_{L}$ and $\tilde{M}_{R}$ are interchanged. Hence, we get

$$
M_{R}=\tilde{A}_{j}^{(2)} \zeta_{j}^{(2)}+\tilde{B}_{j}^{(2)}, \quad j=1,2,4,
$$


where

$$
\tilde{A}_{j}^{(2)}=-\frac{\sqrt{\tilde{\Delta}_{2 j}^{(2)} \Delta_{2 j}^{(2)}}}{\left(m_{\tilde{\chi}_{j}^{ \pm}}^{2}-|\mu|^{2}\right)} \frac{m_{\tilde{\chi}_{j}^{ \pm}}}{\tilde{\mathcal{D}}_{j}^{(2)}},
$$

with

$$
\begin{aligned}
\Delta_{2 j}^{(2)} & =\left(|\mu|^{2}-m_{\tilde{\chi}_{j}^{ \pm}}^{2}\right)\left\{\left(|\mu|^{2}-m_{\tilde{\chi}_{j}^{ \pm}}^{2}\right)\left(\left|M_{L}\right|^{2}-m_{\tilde{\chi}_{j}^{ \pm}}^{2}\right)\right. \\
& -2 \cos ^{2} \theta_{k}\left|\tilde{M}_{R}\right|^{2}\left(m_{\tilde{\chi}_{j}^{ \pm}}^{2}-\left|M_{L}\right|^{2}-2\left|\tilde{M}_{L}\right|^{2} \sin ^{2} \theta_{k}\right) \\
& -2\left|\tilde{M}_{L}\right|^{2} \\
& \times\left[m_{\tilde{\chi}_{j}^{ \pm}}^{2}-\left|M_{L}\right||\mu| \cos \left(2 \tilde{\Phi}_{L}-\Phi_{\mu}-\Phi_{L}\right) \sin \left(2 \theta_{k}\right)\right] \\
& \left.+\left|\tilde{M}_{L}\right|^{4} \sin ^{2}\left(2 \theta_{k}\right)\right\}, \\
\tilde{\Delta}_{2 j}^{(2)} & =\left(|\mu|^{2}-m_{\tilde{\chi}_{j}^{ \pm}}^{2}\right)\left\{\left(|\mu|^{2}-m_{\tilde{\chi}_{j}^{ \pm}}^{2}\right)\left(\left|M_{L}\right|^{2}-m_{\tilde{\chi}_{j}^{ \pm}}^{2}\right)\right. \\
& -2 \sin ^{2} \theta_{k}\left|\tilde{M}_{R}\right|^{2}\left(m_{\tilde{\chi}_{j}^{ \pm}}^{2}-\left|M_{L}\right|^{2}-2\left|\tilde{M}_{L}\right|^{2} \cos ^{2} \theta_{k}\right) \\
& -2\left|\tilde{M}_{L}\right|^{2} \\
& \times\left[m_{\tilde{\chi}_{j}^{ \pm}}^{2}-\left|M_{L}\right||\mu| \cos \left(2 \tilde{\Phi}_{L}-\Phi_{\mu}-\Phi_{L}\right) \sin \left(2 \theta_{k}\right)\right] \\
& \left.+\left|\tilde{M}_{L}\right|^{4} \sin ^{2}\left(2 \theta_{k}\right)\right\},
\end{aligned}
$$

and

$$
\begin{aligned}
\tilde{B}_{j}^{(2)} & =\frac{\tilde{M}_{R}^{2} \sin \left(2 \theta_{k}\right)}{\tilde{\mathcal{D}}_{j}^{(2)}}\left[\left(m_{\tilde{\chi}_{j}^{ \pm}}^{2}-\left|M_{L}\right|^{2}\right)|\mu| e^{-i \Phi_{\mu}}\right. \\
& \left.-M_{L}\left|\tilde{M}_{L}\right|^{2} e^{-2 i \tilde{\Phi}_{L}} \sin \left(2 \theta_{k}\right)\right],
\end{aligned}
$$

where

$$
\begin{aligned}
\tilde{\mathcal{D}}_{j}^{(2)} & =\left|\tilde{M}_{L}\right|^{4} \sin ^{2}\left(2 \theta_{k}\right)+\left(m_{\tilde{\chi}_{j}^{ \pm}}^{2}-\left|M_{L}\right|^{2}\right)\left(m_{\tilde{\chi}_{j}^{ \pm}}^{2}-|\mu|^{2}\right) \\
& -2\left|\tilde{M}_{L}\right|^{2} \\
& \times\left[m_{\tilde{\chi}_{j}^{ \pm}}^{2}-|\mu|\left|M_{L}\right| \cos \left(\Phi_{L}-2 \tilde{\Phi}_{L}+\Phi_{\mu}\right) \sin \left(2 \theta_{k}\right)\right] .
\end{aligned}
$$

Formulas (6.107-6.112) expressing $M_{R}$ in terms of the redefined eigenphases $\zeta_{j}^{(2)}$ and the rest of fundamental parameters may be obtained using a generalized projector formalism method as well. This method will be explained elsewhere.

Note that $\tilde{A}_{j}^{(2)}$ and $\tilde{\mathcal{D}}_{j}^{(2)}$ are real quantities and, as $M_{R}$ has been chosen from the start to be a real parameter, then in the $\mathrm{CP}$-violating case Eq. (6.107) implies a constraint for $\tan \theta_{k}$. Indeed, writing $\zeta_{j}^{(2)}=$ $e^{-i\left(\theta_{2 j}+\tilde{\theta}_{2 j}\right)}=e^{i \Theta_{2 j}}$, we get

$$
M_{R}=\tilde{A}_{j}^{(2)} \cos \Theta_{2 j}+\operatorname{Im}\left(\tilde{B}_{j}^{(2)}\right),
$$

where

$$
\sin \Theta_{2 j}=-\frac{\operatorname{Im}\left(\tilde{B}_{j}^{(2)}\right)}{\tilde{A}_{j}^{(2)}},
$$


with

$$
\begin{aligned}
\operatorname{Re}\left(\tilde{B}_{j}^{(2)}\right) & =\frac{\left|\tilde{M}_{R}\right|^{2} \sin \left(2 \theta_{k}\right)}{\tilde{\mathcal{D}}_{j}^{(2)}}\left[\left(m_{\tilde{\chi}_{j}^{ \pm}}^{2}-\left|M_{L}\right|^{2}\right)|\mu| \cos \left(2 \tilde{\Phi}_{R}-\Phi_{\mu}\right)\right. \\
& \left.-\left|M_{L}\right|\left|\tilde{M}_{L}\right|^{2} \cos \left(2 \tilde{\Phi}_{R}-2 \tilde{\Phi}_{L}+\Phi_{L}\right) \sin \left(2 \theta_{k}\right)\right]
\end{aligned}
$$

and

$$
\begin{aligned}
\operatorname{Im}\left(\tilde{B}_{j}^{(2)}\right) & =\frac{\left|\tilde{M}_{R}\right|^{2} \sin \left(2 \theta_{k}\right)}{\tilde{\mathcal{D}}_{j}^{(2)}}\left[\left(m_{\tilde{\chi}_{j}^{ \pm}}^{2}-\left|M_{L}\right|^{2}\right)|\mu| \sin \left(2 \tilde{\Phi}_{R}-\Phi_{\mu}\right)\right. \\
& \left.-\left|M_{L}\right|\left|\tilde{M}_{L}\right|^{2} \sin \left(2 \tilde{\Phi}_{R}-2 \tilde{\Phi}_{L}+\Phi_{L}\right) \sin \left(2 \theta_{k}\right)\right] .
\end{aligned}
$$

Note that combining Eqs. (6.113) and (6.114) we obtain a quadratic equation determining $M_{R}$, namely

$$
M_{R}^{2}-2 M_{R} \operatorname{Re}\left(\tilde{B}_{j}^{(2)}\right)+\left(\left|\tilde{B}_{j}^{(2)}\right|\right)^{2}-\left(\tilde{A}_{j}^{(2)}\right)^{2}=0
$$

where the solutions are given by

$$
M_{R}= \pm \sqrt{\left(\tilde{A}_{j}^{(2)}\right)^{2}-\left(\operatorname{Im}\left(\tilde{B}_{j}^{(2)}\right)\right)^{2}}+\operatorname{Re}\left(\tilde{B}_{j}^{(2)}\right),
$$

with the constraint $\left(\tilde{A}_{j}^{(2)}\right)^{2}-\left(\operatorname{Im}\left(\tilde{B}_{j}^{(2)}\right)\right)^{2} \geq 0$.

Equation (6.117) can be compared with a similar one found for $M_{2}$ in the context of the MSSM $[16,13]$. Indeed, using the present method in the context of the MSSM, we get $j=1,2$

$$
M_{2}^{2}-2 M_{2} \operatorname{Re}\left(\tilde{b}_{j}^{(1)}\right)+\left(\left|\tilde{b}_{j}^{(1)}\right|\right)^{2}-\left(\tilde{a}_{j}^{(1)}\right)^{2}=0
$$

where

$$
\tilde{a}_{j}^{(1)}=\frac{\sqrt{\tilde{\delta}_{1 j}^{(1)} \delta_{1 j}^{(1)}} m_{\tilde{\chi}_{j}^{ \pm}}}{|\mu|^{2}-m_{\tilde{\chi}_{j}^{ \pm}}^{2}}
$$

with

$$
\delta_{j}^{(1)}=|\mu|^{2}-m_{\tilde{\chi}_{j}^{ \pm}}^{2}+2 m_{W}^{2} \cos ^{2} \beta
$$

and

$$
\tilde{\delta}_{j}^{(1)}=|\mu|^{2}-m_{\tilde{\chi}_{j}^{ \pm}}^{2}+2 m_{W}^{2} \sin ^{2} \beta,
$$

and

$$
\begin{aligned}
\operatorname{Re}\left(\tilde{b}_{j}^{(1)}\right) & =\frac{m_{W}^{2}|\mu| \sin (2 \beta) \cos \Phi_{\mu}}{|\mu|^{2}-m_{\tilde{\chi}_{j}^{ \pm}}^{2}}, \\
\operatorname{Im}\left(\tilde{b}_{j}^{(1)}\right) & =-\frac{m_{W}^{2}|\mu| \sin (2 \beta) \sin \Phi_{\mu}}{|\mu|^{2}-m_{\tilde{\chi}_{j}^{ \pm}}^{2}} .
\end{aligned}
$$

Therefore, as before, from Eq. (6.119), the solutions for $M_{2}$ are given by

$$
M_{2}= \pm \sqrt{\left(\tilde{a}_{j}^{(1)}\right)^{2}-\left(\operatorname{Im}\left(\tilde{b}_{j}^{(1)}\right)\right)^{2}}+\operatorname{Re}\left(\tilde{b}_{j}^{(1)}\right),
$$


with the constraint $\left(\tilde{a}_{j}^{(1)}\right)^{2}-\left(\operatorname{Im}\left(\tilde{b}_{j}^{(1)}\right)\right)^{2} \geq 0$.

We note that in the L-R SUSY model, $M_{R}$ becomes singular at the points where either $\left(|\mu|^{2}-m_{\tilde{\chi}_{j}^{ \pm}}^{2}\right)$ or $\tilde{\mathcal{D}}_{j}^{(2)}$ vanishes whereas in the MSSM the singularities of $M_{2}$ are only present at the point where $\left(|\mu|^{2}-m_{\tilde{\chi}_{j}^{ \pm}}^{2}\right)$ vanishes. Apart from that, the role played by $M_{R}, \mu$ and $\tan \theta_{k}$ in the chargino sector in the context of the L-R SUSY model, when the remaining parameters are fixed, is similar to the role played by $M_{2}, \mu$ and $\tan \beta$ in the chargino sector in the context of the MSSM. This suggests us to make use of some useful technics implemented in the literature $[16,20,23,24,25]$ in the context of the MSSM, concerning to the measurement of some independent cross section physical observables related to the chargino and neutralino pair production in $e^{+} e^{-}$annihilation processes, that could be applied in the context of the L-R SUSY model to fix the chargino and neutralino parameters.

\section{Determining L-R SUSY parameters}

In this section we give some examples on the analytical and numerical reconstruction of the fundamental L-R SUSY parameters of the ino sector by considering some possible $\mathrm{CP}$-conserving and $\mathrm{CP}$-violating scenarios.

\subsection{Scenario $S c p v_{3}$}

Let us first to consider the scenario $S c p v_{3}$ given in Tab. 4, where the L-R SUSY parameters $M_{R}, \tan \theta_{k}$ and $|\mu|$ are known and where the phases $\Phi_{\mu}=\tilde{\Phi}_{L}=\tilde{\Phi}_{R}=0$. As before, we assume that $g_{L}=g_{R}=0.65$, and $k_{u}=92.75$.

Figures 8 and 9 show the values of $\left(\left|M_{L}\right|-m_{\tilde{\chi}_{j}^{ \pm}}\right) / 2$ predicted by Eq. (6.97) as a function of the chargino physical masses $m_{\tilde{\chi}_{j}^{ \pm}}$for different values of the eigenphase $\operatorname{argument} \operatorname{Arg}\left(\xi_{j}\right)=0, \pi / 4, \pi / 2,3 \pi / 4, \pi$, under the conditions described in scenario $S c p v_{3}$ with $\tan \theta_{k}=4$ and $\tan \theta_{k}=30$, respectively. Comparing these figures we observe that for a given value of the chargino mass, the variation of $|M|_{L}-m_{\tilde{\chi}_{j}^{ \pm}}$, with respect to the argument of the eigenphases $\xi_{j}$ when the ratio $\tan \theta_{k}=k_{u} / k_{d}$ is small is greater than the variation of this quantity when this ratio is large. In the case where $\tan \theta_{k}=4$, we observe that for values of $m_{\tilde{\chi}_{j}^{ \pm}} \approx 470 \mathrm{GeV}$, the values of the parameter $\left|M_{L}\right|$ are approximately the same $(\sim 470 \mathrm{GeV})$, whereas for values of $m_{\tilde{\chi}_{j}^{ \pm}} \approx 160 \mathrm{GeV}$, the values of this parameter lie in the range of $160 \mathrm{GeV}-167 \mathrm{GeV}$, approximately. The singularities in the plot of Fig. 8 occurs when the factor $\left(m_{\tilde{\chi}_{j}^{ \pm}}^{2}-|\mu|^{2}\right)$ in the denominator of Eq. (6.98) vanishes, i.e., when $m_{\tilde{\chi}_{j}^{ \pm}}=248 \mathrm{GeV}$ and also when the factor $\tilde{\mathcal{D}}_{j}=0$ in the denominator of Eqs. (6.98) and (6.99) vanishes, i.e., when $m_{\tilde{\chi}_{j}^{ \pm}} \approx 247.7 \mathrm{GeV}$ and $m_{\tilde{\chi}_{j}^{ \pm}} \approx 498.6 \mathrm{GeV}$. By the same reasons, the singularities in the plot of Fig. 9 occurs at the points $m_{\tilde{\chi}_{j}^{ \pm}}=248 \mathrm{GeV}, m_{\tilde{\chi}_{j}^{ \pm}}=246 \mathrm{GeV}$ and $m_{\tilde{\chi}_{j}^{ \pm}}=498.4 \mathrm{GeV}$, respectively. On the other hand, contrary to the case of the neutralino [11], in the case where $\tan \theta_{k}=30$, we don't observe a distinguishable value of the chargino mass where the different curves intersect, i.e., the corresponding graph don't allows us to determine an optimal value of $\left|M_{L}\right|$. 


$\begin{array}{cccccc}\text { Scenario } & |\mu| & M_{R} & \Phi_{\mu}=\tilde{\Phi}_{L}=\tilde{\Phi}_{R} & \tan \theta_{k} & \operatorname{Arg}\left(\xi_{j}\right) \\ & & & & \\ & & & & & 0 \\ \text { Spvc }_{3} & 248 & 500 & 0 & 4 & \pi / 4 \\ & & & & 30 & \pi / 2 \\ & & & & & \pi \\ \end{array}$

Table 4: Input parameters for scenario $S c p v_{3}$. All mass quantities are in GeV.

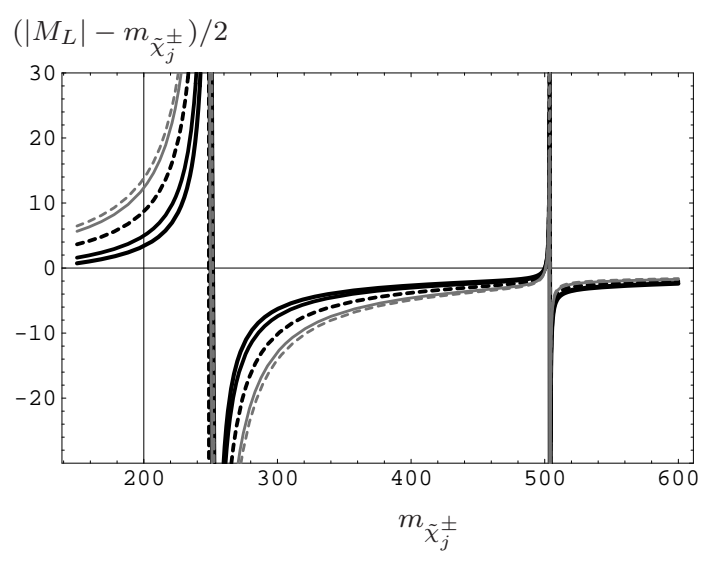

Figure 8: Reconstruction of $\left|M_{L}\right|$ as a function of the physical chargino masses $m_{\tilde{\chi}_{j}^{ \pm}}$, using the general formula (6.97), for inputs of scenario $S c p v_{3}$ with $\tan \theta_{k}=4$. The curves are: $\operatorname{Arg}\left(\xi_{j}\right)=0$ (heavy solid), $\operatorname{Arg}\left(\xi_{j}\right)=\pi / 4$ (solid), $\operatorname{Arg}\left(\xi_{j}\right)=\pi / 2$ (dashed), $\operatorname{Arg}\left(\xi_{j}\right)=3 \pi / 4$ (light solid), $\operatorname{Arg}\left(\xi_{j}\right)=\pi$ (light dashed).

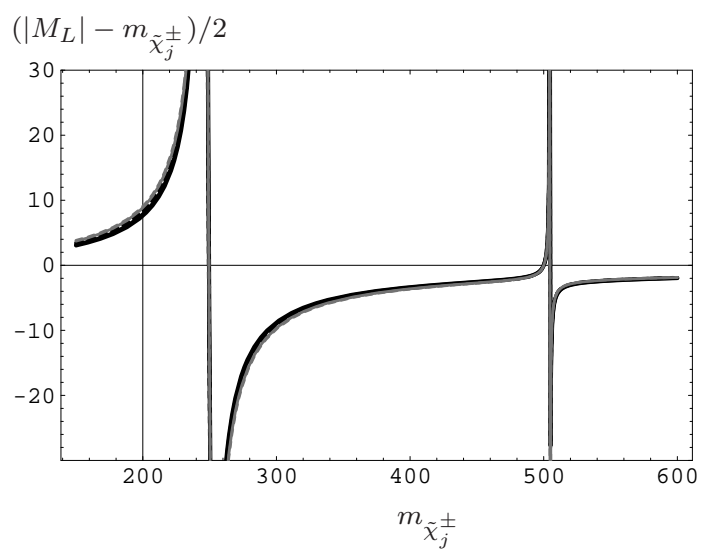

Figure 9: The same as in Fig. 8 but considering $\tan \theta_{k}=30$. 


\subsection{Scenario $\mathrm{Scpv}_{4}$}

Let us now to consider a scenario where the physical mass of two lightest charginos, $m_{\tilde{\chi}_{1}^{ \pm}}$and $m_{\tilde{\chi}_{2}^{ \pm}}$are known. Then, from Eq. (6.97), the parameters $\left|M_{L}\right|$ and $\Phi_{L}$ and the eigenphases $\zeta_{1}$ and $\zeta_{2}$, can be expressed analytically, up to a twofold discrete ambiguity, in terms of parameters $|\mu|, \Phi_{\mu}, M_{R}, \tan \theta_{k}, \tilde{\Phi}_{R}$ and $\tilde{\Phi}_{L}$. Indeed, following the same treatment as in the case of the neutralino in the MSSM[13], if the two lightest chargino masses are known, then from Eq. (6.97), we deduce the following expression for the chargino eigenphases

$$
\zeta_{1,2}=\frac{\tilde{A}_{1}^{2}-\tilde{A}_{2}^{2} \pm|\Delta \tilde{B}|^{2}+i \epsilon \sqrt{\tilde{\Delta}_{c h}}}{2 \tilde{A}_{1,2} \Delta \tilde{B}^{*}}
$$

where

$$
\begin{gathered}
\Delta \tilde{B}=\tilde{B}_{2}-\tilde{B}_{1} \\
\tilde{\Delta}_{c h}=4 \tilde{A}_{1}^{2} \tilde{A}_{2}^{2}-\left(\tilde{A}_{1}^{2}+\tilde{A}_{2}^{2}-|\Delta \tilde{B}|^{2}\right)^{2} \geq 0
\end{gathered}
$$

and $\epsilon= \pm 1$. Then, inserting Eq. (7.126) into (6.97) we get

$$
M_{L}=\frac{\tilde{A}_{1}^{2}-\tilde{A}_{2}^{2} \pm\left(\tilde{B}_{1}+\tilde{B}_{2}\right) \Delta \tilde{B}+i \epsilon \sqrt{\tilde{\Delta}_{c h}}}{2 \Delta \tilde{B}^{*}} .
$$

On the other hand, under similar conditions, we can give an equivalent description to the one given above. Indeed, writing $\tilde{\mathcal{B}}_{j}, j=1,2$, in the form

$$
\tilde{\mathcal{B}}_{j}=\frac{\tilde{\mathcal{P}}_{j}+\tilde{\mathcal{Q}}_{j} \tan \left(\Phi_{L}-2 \tilde{\Phi}_{L}\right)}{\sqrt{1+\tan ^{2}\left(\Phi_{L}-2 \tilde{\Phi}_{L}\right)}}
$$

with

$$
\begin{aligned}
\tilde{\mathcal{P}}_{j} & =2\left|\tilde{M}_{L}\right|^{2} \sin \left(2 \theta_{k}\right) \\
& \times\left[M_{R}\left|\tilde{M}_{R}\right|^{2} \cos \left(2 \tilde{\Phi}_{R}\right) \sin \left(2 \theta_{k}\right)\right. \\
& \left.-|\mu|\left(m_{\tilde{\chi}_{j}^{ \pm}}^{2}-M_{R}^{2}\right) \cos \Phi_{\mu}\right]
\end{aligned}
$$

and

$$
\begin{aligned}
\tilde{\mathcal{Q}}_{j} & =2\left|\tilde{M}_{L}\right|^{2} \sin \left(2 \theta_{k}\right) \\
& \times\left[-M_{R}\left|\tilde{M}_{R}\right|^{2} \sin \left(2 \tilde{\Phi}_{R}\right) \sin \left(2 \theta_{k}\right)\right. \\
& \left.+|\mu|\left(m_{\tilde{\chi}_{j}^{ \pm}}^{2}-M_{R}^{2}\right) \sin \Phi_{\mu}\right],
\end{aligned}
$$

and inserting it into Eq. (6.106), after some algebraic manipulations we get

$$
\tan \left(\Phi_{L}-2 \tilde{\Phi}_{L}\right)=\tilde{\mathbb{R}} \equiv \frac{-\tilde{\mathbb{B}}-\tilde{\epsilon} \sqrt{\tilde{\mathbb{B}}^{2}-4 \tilde{\mathbb{A}}}}{2 \tilde{\mathbb{C}}}
$$

where $\tilde{\mathbb{B}}^{2}-4 \tilde{\mathbb{A}} \tilde{\mathbb{C}} \geq 0$,

$$
\tilde{\mathbb{A}}=\frac{1}{2} F\left(\tilde{\mathcal{Q}}_{1}, \tilde{\mathcal{Q}}_{2}, \tilde{\mathcal{Q}}_{1}, \tilde{\mathcal{Q}}_{2}\right)-\left(\tilde{\mathcal{D}}_{1} \tilde{\mathcal{C}}_{2}-\tilde{\mathcal{D}}_{2} \tilde{\mathcal{C}}_{1}\right)^{2}
$$




\begin{tabular}{llllllll} 
Scenario & $|\mu|$ & $M_{R}$ & $m_{\tilde{\chi}_{1}^{ \pm}}$ & $m_{\tilde{\chi}_{2}^{ \pm}}$ & $\tan \theta_{k}$ & $\tilde{\Phi}_{R}$ \\
\cline { 1 - 5 } & & & & & \\
$S_{S c p v_{4}}$ & 248 & 300 & 158.5 & $\begin{array}{l}247.30 \\
247.38\end{array}$ & 30 & 0 \\
\hline
\end{tabular}

Table 5: Input parameters for scenario $S c p v_{4}$. All mass quantities are in $\mathrm{GeV}$.

$$
\tilde{\mathbb{B}}=F\left(\tilde{\mathcal{P}}_{1}, \tilde{\mathcal{P}}_{2}, \tilde{\mathcal{Q}}_{1}, \tilde{\mathcal{Q}}_{2}\right)
$$

and

$$
\tilde{\mathbb{C}}=\frac{1}{2} F\left(\tilde{\mathcal{P}}_{1}, \tilde{\mathcal{P}}_{2}, \tilde{\mathcal{P}}_{1}, \tilde{\mathcal{P}}_{2}\right)-\left(\tilde{\mathcal{D}}_{1} \tilde{\mathcal{C}}_{2}-\tilde{\mathcal{D}}_{2} \tilde{\mathcal{C}}_{1}\right)^{2}
$$

with

$$
\begin{aligned}
F\left(\tilde{\mathcal{P}}_{1}, \tilde{\mathcal{P}}_{2}, \tilde{\mathcal{Q}}_{1}, \tilde{\mathcal{Q}}_{2}\right)= & \left(\tilde{\mathcal{D}}_{1} \tilde{\mathcal{C}}_{2}+\tilde{\mathcal{D}}_{2} \tilde{\mathcal{C}}_{1}\right)\left(\tilde{\mathcal{P}}_{1} \tilde{\mathcal{Q}}_{2}+\tilde{\mathcal{P}}_{2} \tilde{\mathcal{Q}}_{1}\right) \\
- & 2\left(\tilde{\mathcal{D}}_{1} \tilde{\mathcal{C}}_{1} \tilde{\mathcal{P}}_{2} \tilde{\mathcal{Q}}_{2}+\tilde{\mathcal{D}}_{2} \tilde{\mathcal{C}}_{2} \tilde{\mathcal{P}}_{1} \tilde{\mathcal{Q}}_{1}\right),
\end{aligned}
$$

where $\tilde{\epsilon}= \pm 1$.

Moreover, combining Eq. (6.103) for $j=1$ with Eq. (6.103) for $j=2$, and then using Eq. (7.130) for $j=1,2$, we get

$$
\left|M_{L}\right|=\frac{\left(\tilde{\mathcal{D}}_{1} \tilde{\mathcal{C}}_{2}-\tilde{\mathcal{D}}_{2} \tilde{\mathcal{C}}_{1}\right) \sqrt{1+\tilde{\mathbb{R}}^{2}}}{\left(\tilde{\mathcal{D}}_{2} \tilde{\mathcal{P}}_{1}-\tilde{\mathcal{D}}_{1} \tilde{\mathcal{P}}_{2}\right)+\left(\tilde{\mathcal{D}}_{2} \tilde{\mathcal{Q}}_{1}-\tilde{\mathcal{D}}_{1} \tilde{\mathcal{Q}}_{2}\right) \tilde{\mathbb{R}}} .
$$

Equations (7.133) and (7.138) allows us to determine the phase difference $\Phi_{L}-2 \tilde{\Phi}_{L}$ and the norm $\left|M_{L}\right|$, respectively, up to a twofold discrete ambiguity, in terms of the two lightest chargino masses and the fundamental parameters $|\mu|, \Phi_{\mu}, M_{R}, \tan \theta_{k}$ and $\tilde{\Phi}_{R}$.

For instance, let us consider the CP-violating scenario $S c p v_{4}$ given in Tab. 5. Figure 10, show the behaviour of $\Phi_{L}-2 \tilde{\Phi}_{L}$ as a function of $\Phi_{\mu}$ when this last parameter is allowed to vary in the interval $[-0.95(\mathrm{rad}), 0.95(\mathrm{rad})]$. This interval correspond approximately to the rang where the discriminant under the root in Eq. (7.133), is greater or equal to zero. The two different curves observed in this figure demonstrate the twofold ambiguity corresponding to the two possible values of $\tilde{\epsilon}= \pm 1$. Figure 11 , show the behaviour of $\left|M_{L}\right|$, computed from Eq.(7.138), as a function of $\Phi_{\mu}$, in the same interval as in Figure 10. The values of $\left|M_{L}\right|$ fluctuate in the range of $166.7 \mathrm{GeV}-167.3 \mathrm{GeV}$ approximately. The two fold ambiguity induced by the two different signs of $\tilde{\epsilon}$ is practically not observed in this case.

The values of the phase angles and $\left|M_{L}\right|$ are very sensible to the change of the physical masses of the charginos. For instance, according to the input parameters of scenario $S c p v_{4}$, if the mass of the second chargino is chosen to be $m_{\tilde{\chi}_{2}^{ \pm}}=247.38 \mathrm{GeV}$, then the values of $\Phi_{\mu}$ where the discriminant under the root in Eq. (7.133) is greater or equal to zero are restricted to the interval [-0.85 rad, $0.85 \mathrm{rad}]$. Also, the values of the phase difference $\Phi_{L}-2 \tilde{\Phi}_{L}$ and $\left|M_{L}\right|$, for a given value of $\Phi_{\mu}$ vary with respect to precedent case, now the values of $\left|M_{L}\right|$ fluctuate in the range of $166.8 \mathrm{GeV}-167.5 \mathrm{GeV}$ approximately, as we can see from Figures 12 and 13, respectively. 


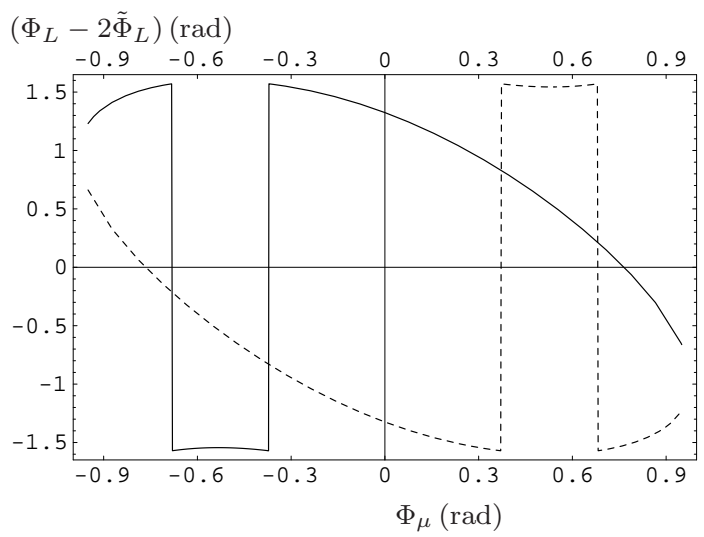

Figure 10: behaviour of $\Phi_{L}-2 \tilde{\Phi}_{L}$ as a function of $\Phi_{\mu}$, computed from Eq. (7.133), for input parameters of scenario $S c p v_{4}$, with $m_{\tilde{\chi}_{2}^{ \pm}}=247.30 \mathrm{GeV}$. The curves are: $\tilde{\epsilon}=1$ (solid), $\tilde{\epsilon}=-1$ (dashed).

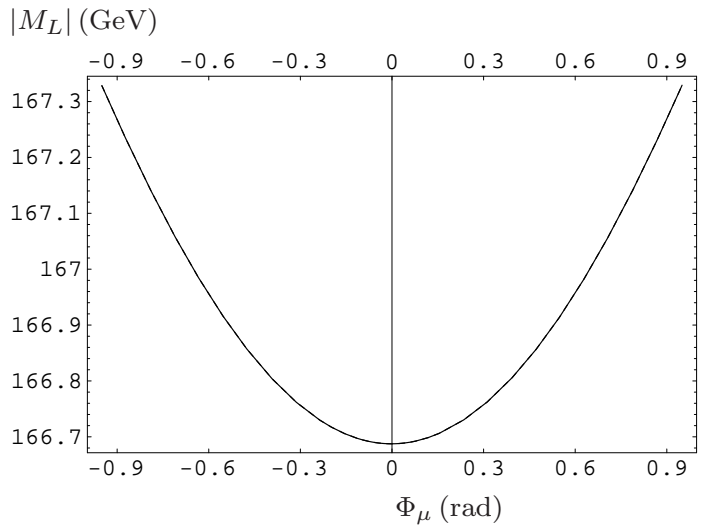

Figure 11: Norm of $M_{L}$ as a function of $\Phi_{\mu}$, computed from Eq. (7.138), for input parameters of scenario $S c p v_{4}$, with $m_{\tilde{\chi}_{2}^{ \pm}}=247.30 \mathrm{GeV}$. The curve are practically the same for $\tilde{\epsilon}=1$ as for $\tilde{\epsilon}=-1$.

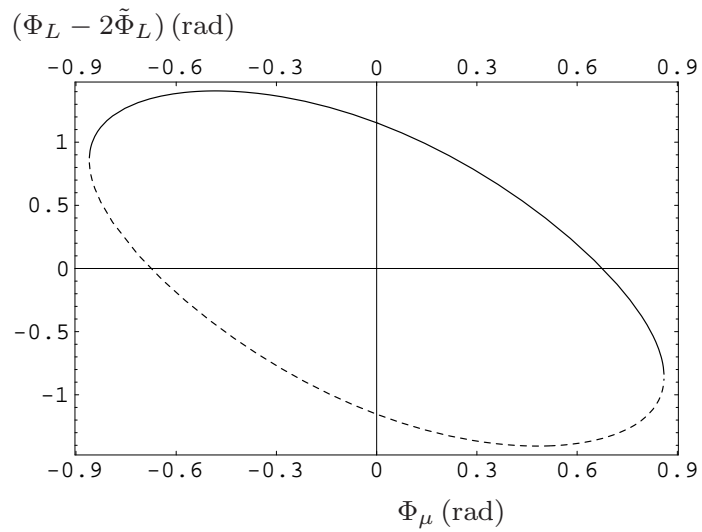

Figure 12: behaviour of $\Phi_{L}-2 \tilde{\Phi}_{L}$ as a function of $\Phi_{\mu}$, computed from Eq. (7.133), for input parameters of scenario $\mathrm{Scpv}_{4}$, with $m_{\tilde{\chi}_{2}^{ \pm}}=247.38 \mathrm{GeV}$. The curves are: $\tilde{\epsilon}=1$ (solid), $\tilde{\epsilon}=-1$ (dashed). 


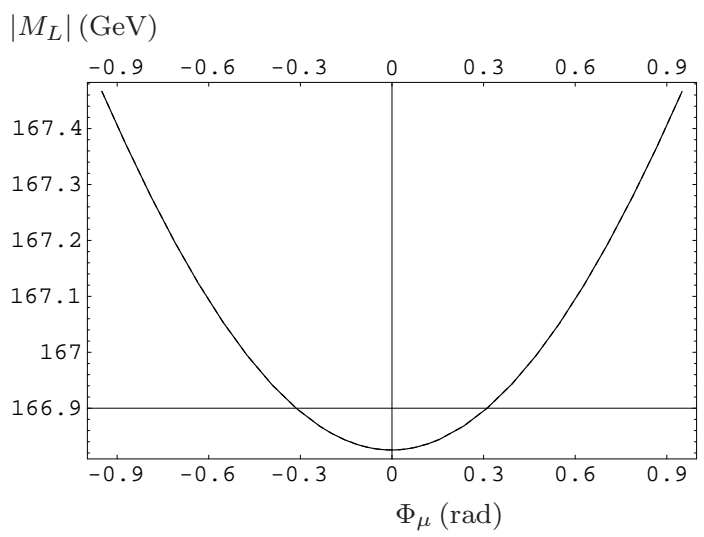

Figure 13: Norm of $M_{L}$ as a function of $\Phi_{\mu}$, computed from Eq. (7.138), for input parameters of scenario $S c p v_{4}$, with $m_{\tilde{\chi}_{2}^{ \pm}}=247.30 \mathrm{GeV}$. The curve are practically the same for $\tilde{\epsilon}=1$ as for $\tilde{\epsilon}=-1$.

\subsection{Scenario $S c p v_{5}$}

Let us assume now that in addition to the two lightest chargino masses $m_{\tilde{\chi}_{1}^{ \pm}}$and $m_{\tilde{\chi}_{2}^{ \pm}}$, we also known the physical masses of the two lightest neutralinos $m_{\tilde{\chi}_{1}^{0}}$ and $m_{\tilde{\chi}_{2}^{0}}$.

In the same way as in Section (6.2), from the neutralino sector of the L-R SUSY model, we can express the norm of $M_{L}$ in terms of the neutralino physical masses $m_{\tilde{\chi}_{j}^{0}}, j=1, \ldots, 4$ and the fundamental parameters $\Phi_{L}, M_{R}, M_{V},|\mu|, \Phi_{\mu}$ and $\tan \theta_{k}$, by solving the algebraic equation [11]

$$
\mathcal{D}_{j}\left|M_{L}\right|^{2}+\mathcal{B}_{j}\left|M_{L}\right|+\mathcal{C}_{j}=0,
$$

where

$$
\begin{aligned}
\mathcal{B}_{j} & =-4\left(M_{W_{L}}\right)^{2}\left\{|\mu|\left(m_{\tilde{\chi}_{j}^{0}}^{2}-M_{R V}^{2}\right)\left(m_{\tilde{\chi}_{j}^{0}}^{2}-4|\mu|^{2}\right)\right. \\
& \times \cos \left(\Phi_{L}+\Phi_{\mu}\right) \sin \left(2 \theta_{k}\right)+2 \kappa^{2}\left(M_{W_{L}}\right)^{2} M_{R V} \\
& \left.\times\left[m_{\tilde{\chi}_{j}^{0}}^{2}-4|\mu|^{2} \sin ^{2}\left(2 \theta_{k}\right)\right] \cos \Phi_{L}\right\} \\
\mathcal{C}_{j} & =m_{\tilde{\chi}_{j}^{0}}^{2}\left(m_{\tilde{\chi}_{j}^{0}}^{2}-M_{R V}^{2}\right)\left(m_{\tilde{\chi}_{j}^{0}}^{2}-4|\mu|^{2}\right)^{2} \\
& -2 m_{\tilde{\chi}_{j}^{0}}^{2}\left(M_{W_{L}}\right)^{2}\left(m_{\tilde{\chi}_{j}^{0}}^{2}-4|\mu|^{2}\right)\left[m_{\tilde{\chi}_{j}^{0}}^{2}\left(1+4 \kappa^{2}\right)\right. \\
& \left.-M_{R V}{ }^{2}+8|\mu| M_{R V} \kappa^{2} \cos \left(\Phi_{\mu}\right) \sin \left(2 \theta_{k}\right)\right] \\
& +\left(M_{W_{L}}\right)^{4}\left[m_{\tilde{\chi}_{j}^{0}}^{2}\left(1+4 \kappa^{2}\right)^{2}-M_{R V}{ }^{2}\right] \\
& \times\left[m_{\tilde{\chi}_{j}^{0}}^{2}-4|\mu|^{2} \sin \left(2 \theta_{k}\right)^{2}\right]
\end{aligned}
$$

and

$$
\begin{aligned}
\mathcal{D}_{j} & =-\left\{\left(m_{\tilde{\chi}_{j}^{0}}^{2}-M_{R V}^{2}\right)\left(m_{\tilde{\chi}_{j}^{0}}^{2}-4|\mu|^{2}\right)^{2}-8 \kappa^{2}\left(M_{W_{L}}\right)^{2}\right. \\
& \times\left(m_{\tilde{\chi}_{j}^{0}}^{2}-4|\mu|^{2}\right)\left[m_{\tilde{\chi}_{j}^{0}}^{2}+2|\mu| M_{R V} \cos \Phi_{\mu} \sin \left(2 \theta_{k}\right)\right] \\
& \left.+16 \kappa^{4}\left(M_{W_{L}}\right)^{4}\left[m_{\tilde{\chi}_{j}^{0}}^{2}-4|\mu|^{2} \sin ^{2}\left(2 \theta_{k}\right)\right]\right\}
\end{aligned}
$$


where $M_{W_{L}}$ is the mass of the left-handed gauge bosons given in Eq. (2.15), $M_{R V}=\left(4 g_{V}^{2} M_{R}+g_{R}^{2} M_{V}\right) / g_{1}^{2}$ and $\kappa=\frac{g_{R} g_{V}}{g_{1} g_{L}}$, where $g_{1}=\left(g_{R}^{2}+4 g_{V}^{2}\right)^{1 / 2}$ and $g_{V}$ is the coupling constant associated to the gauge group $U(1)_{B-L}$.

Thus, writing $\mathcal{B}_{j}, j=1,2$, in the form

$$
\mathcal{B}_{j}=\frac{\mathcal{P}_{j}+\mathcal{Q}_{j} \tan \Phi_{L}}{\sqrt{1+\tan ^{2} \Phi_{L}}}
$$

with

$$
\begin{aligned}
\mathcal{P}_{j} & =-4\left(M_{W_{L}}\right)^{2}\left\{|\mu|\left(m_{\tilde{\chi}_{j}^{0}}^{2}-M_{R V}^{2}\right)\left(m_{\tilde{\chi}_{j}^{0}}^{2}-4|\mu|^{2}\right)\right. \\
& \times \cos \Phi_{\mu} \sin \left(2 \theta_{k}\right)+2 \kappa^{2}\left(M_{W_{L}}\right)^{2} M_{R V} \\
& \left.\times\left[m_{\tilde{\chi}_{j}^{0}}^{2}-4|\mu|^{2} \sin ^{2}\left(2 \theta_{k}\right)\right]\right\}
\end{aligned}
$$

and

$$
\begin{aligned}
\mathcal{Q}_{j} & =4|\mu|\left(M_{W_{L}}\right)^{2}\left(m_{\tilde{\chi}_{j}^{0}}^{2}-M_{R V}^{2}\right)\left(m_{\tilde{\chi}_{j}^{0}}^{2}-4|\mu|^{2}\right) \\
& \times \sin \Phi_{\mu} \sin \left(2 \theta_{k}\right)
\end{aligned}
$$

and proceeding as in the previous subsection, we get

$$
\tan \Phi_{L}=\mathbb{R} \equiv \frac{-\mathbb{B}-\epsilon \sqrt{\mathbb{B}^{2}-4 \mathbb{A} \mathbb{C}}}{2 \mathbb{A},}
$$

where $\epsilon= \pm 1, \mathbb{B}^{2}-4 \mathbb{A} \mathbb{C} \geq 0$, and $\mathbb{A}, \mathbb{B}$ and $\mathbb{C}$ are obtained from Eqs. (7.134), (7.135) and (7.136), respectively, by removing the tilde on the variables.

Moreover, as in the precedent subsection, we also get

$$
\left|M_{L}\right|=\frac{\left(\mathcal{D}_{1} \mathcal{C}_{2}-\mathcal{D}_{2} \mathcal{C}_{1}\right) \sqrt{1+\mathbb{R}^{2}}}{\left(\mathcal{D}_{2} \mathcal{P}_{1}-\mathcal{D}_{1} \mathcal{P}_{2}\right)+\left(\mathcal{D}_{2} \mathcal{Q}_{1}-\mathcal{D}_{1} \mathcal{Q}_{2}\right) \mathbb{R}}
$$

Equations (7.145) and (7.146) express the phase $\Phi_{L}$ and norm $\left|M_{L}\right|$, in terms of the two lightest neutralino masses and the parameters $|\mu|, \Phi_{\mu}, M_{R}, M_{V}, \tan \theta_{k}$, respectively.

Now, combining Eqs. (7.133) and (7.145), we obtain an expression for the chargino mixing angle $\tilde{\Phi}_{L}$, in terms of the two lightest neutralino physical masses, the two lightest chargino physical masses and the set of parameters described previously in addition to the mixing angle $\tilde{\Phi}_{R}$, namely,

$$
\tan \left(2 \tilde{\Phi}_{L}\right)=\frac{\mathbb{R}-\tilde{\mathbb{R}}}{1+\mathbb{R} \tilde{\mathbb{R}}},
$$

where $\tilde{\mathbb{R}}\left(m_{\tilde{\chi}_{1}^{ \pm}}, m_{\tilde{\chi}_{2}^{ \pm}},|\mu|, \Phi_{\mu}, M_{R}, \tilde{\Phi}_{R}, \tan \theta_{k}\right)$ and $\mathbb{R}\left(m_{\tilde{\chi}_{1}^{0}}, m_{\tilde{\chi}_{2}^{0}},|\mu|, \Phi_{\mu}, M_{R}, M_{V}, \tan \theta_{k}\right)$ are given by the right side member of Eqs. (7.133) and (7.145), respectively. Moreover, equating Eqs. (7.138) and (7.146), we obtain an equation serving to determine, at least numerically, one of the remaining parameters. 


\subsubsection{Case $\tilde{\Phi}_{L}=0$}

In the case $\tilde{\Phi}_{L}=0$, from Eq. (7.147) we get $\tilde{\mathbb{R}}=\mathbb{R}$ and, taking in account this result, combining (7.138) and (7.146), after some manipulations we obtain

$$
\tilde{\mathbb{R}}=\mathbb{R}=-\frac{g\left[\mathcal{P}_{1}, \mathcal{P}_{2}, \tilde{\mathcal{P}}_{1}, \tilde{\mathcal{P}}_{2}\right]}{g\left[\mathcal{Q}_{1}, \mathcal{Q}_{2}, \tilde{\mathcal{Q}}_{1}, \tilde{\mathcal{Q}}_{2}\right]},
$$

where

$$
g\left[\mathcal{P}_{1}, \mathcal{P}_{2}, \tilde{\mathcal{P}}_{1}, \tilde{\mathcal{P}}_{2}\right]=\left(\mathcal{D}_{1} \mathcal{C}_{2}-\mathcal{D}_{2} \mathcal{C}_{1}\right)\left(\tilde{\mathcal{D}}_{1} \tilde{\mathcal{P}}_{2}-\tilde{\mathcal{D}}_{2} \tilde{\mathcal{P}}_{1}\right)-\left(\tilde{\mathcal{D}}_{1} \tilde{\mathcal{C}}_{2}-\tilde{\mathcal{D}}_{2} \tilde{\mathcal{C}}_{1}\right)\left(\mathcal{D}_{1} \mathcal{P}_{2}-\mathcal{D}_{2} \mathcal{P}_{1}\right)
$$

The equality $\tilde{\mathbb{R}}=\mathbb{R}$ in Eq. (7.148) produces an algebraic equation relating the parameters $M_{R}, M_{V}$, $|\mu|, \Phi_{\mu}, \tilde{\Phi}_{R}$ and $\tan \theta_{k}$, namely

$$
(\mathbb{A} \tilde{\mathbb{C}}-\mathbb{C} \tilde{\mathbb{A}})^{2}-(\mathbb{B} \tilde{\mathbb{C}}-\mathbb{C} \tilde{\mathbb{B}})(\mathbb{A} \tilde{\mathbb{B}}-\mathbb{B} \tilde{\mathbb{A}})=0,
$$

whereas the equality of $\mathbb{R}$ with the third member in Eq. (7.148) leads to the following algebraic equation for these parameters:

$$
\mathbb{A} g\left[\mathcal{P}_{1}, \mathcal{P}_{2}, \tilde{\mathcal{P}}_{1}, \tilde{\mathcal{P}}_{2}\right]^{2}-\mathbb{B} g\left[\mathcal{P}_{1}, \mathcal{P}_{2}, \tilde{\mathcal{P}}_{1}, \tilde{\mathcal{P}}_{2}\right] g\left[\mathcal{Q}_{1}, \mathcal{Q}_{2}, \tilde{\mathcal{Q}}_{1}, \tilde{\mathcal{Q}}_{2}\right]+\mathbb{C} g\left[\mathcal{Q}_{1}, \mathcal{Q}_{2}, \tilde{\mathcal{Q}}_{1}, \tilde{\mathcal{Q}}_{2}\right]^{2}=0
$$

For fixed $M_{V},|\mu|, \Phi_{\mu}$ and $\tilde{\Phi}_{R}$, Equations (7.150) and (7.151) represent a system of equations serving to determine, at least numerically, $M_{R}$ and $\tan \theta_{k}$. Indeed, (7.150) corresponds to an algebraic equation of order 32 in the variables $M_{R}$ and $\sin \left(2 \theta_{k}\right)$ whereas (7.151), which can be factorized by $\left(\mathcal{D}_{1} \mathcal{C}_{2}-\mathcal{D}_{2} \mathcal{C}_{1}\right)^{2}$, corresponds to an algebraic equation of order 16 in these variables. Clearly, the analytical treatment to obtain the solutions of these equations is hard, the order of them is too large, however numerical extraction of solutions shouldn't represent, in principle, any problem. Apart from the fact that the solutions have to verify Eqs. (7.150-7.150), they also must verify the constraints described above, namely $\tilde{\mathbb{B}}^{2}-4 \tilde{\mathbb{A}} \tilde{\mathbb{C}} \geq 0$, $\mathbb{B}^{2}-4 \mathbb{A} \mathbb{C} \geq 0, \tilde{\mathcal{B}}_{j}^{2}-4 \tilde{\mathcal{D}}_{j} \tilde{\mathcal{C}}_{j} \geq 0, \tilde{\mathcal{B}}_{j} / \tilde{\mathcal{D}}_{j}<0, \mathcal{B}_{j}^{2}-4 \mathcal{D}_{j} \mathcal{C}_{j} \geq 0$, and $\mathcal{B}_{j} / \mathcal{D}_{j}<0$, this fact reduce considerably the number of possible solutions.

\section{Disentangle of the chargino sector L-R SUSY parameters based on cross- section physical observables}

Further independent relations serving determine the fundamental parameters can be obtained by computing some physical observables such as the total cross section of the chargino pair production in $e^{+} e^{-}$annihilation, Left-Right asymmetries and polarization vectors, similarly as it has been in the case of the MSSM [23]. In this section, we give an outline of the general procedure that we could follow to fix the fundamental L-R SUSY based on experimental measurements of cross-section-type observables.

The production of the chargino pairs at $e^{+} e^{-}$colliders in the context of the L-R SUSY model at the tree level arise from $e^{+} e^{-} \rightarrow \gamma, Z_{L}, Z_{R} \rightarrow \tilde{\chi}_{i}^{+} \tilde{\chi}_{j}^{-}$in the s-channel, and $e^{+} e^{-} \rightarrow \tilde{\nu}_{L, R} \rightarrow \tilde{\chi}_{i}^{+} \tilde{\chi}_{j}^{-}$in the 
t-channel. The Lagrangian corresponding to these interactions is given by $[15,18]$

$$
\begin{aligned}
\mathcal{L} & =-e A_{\mu} \overline{\tilde{\chi}_{i}^{+}} \gamma^{\mu} \tilde{\chi}_{j}^{+}+\frac{g_{L}}{\cos \theta_{W}} Z_{L}^{\mu} \overline{\tilde{\chi}_{i}^{+}} \gamma^{\mu}\left(O_{i j}^{\prime L} \gamma_{L}+O_{i j}^{\prime R} \gamma_{R}\right) \tilde{\chi}_{j}^{+} \\
& +\frac{g_{R} \sqrt{\cos \left(2 \theta_{W}\right)}}{\cos \theta_{W}} Z_{R}^{\mu} \overline{\tilde{\chi}_{i}^{+}} \gamma^{\mu}\left(O_{i j}^{L} \gamma_{L}+O_{i j}^{R} \gamma_{R}\right) \tilde{\chi}_{j}^{+} \\
& -g \sum_{\ell=i, j} \overline{\tilde{\chi}_{\ell}^{+}}\left\{\left[V_{1 \ell} \gamma_{L}-\left(\frac{m_{\nu_{s}} U_{3 \ell}^{*}}{\sqrt{2} m_{W} \sin \theta_{k}}+\frac{m_{e_{s}} U_{4 \ell}^{*}}{\sqrt{2} m_{W} \cos \theta_{k}}\right) \gamma_{R}\right] e_{s} \tilde{\nu}_{L s}^{*}\right. \\
& \left.+\left[U_{2 \ell}^{*} \gamma_{R}-\left(\frac{m_{\nu_{s}} V_{3 \ell}}{\sqrt{2} m_{W} \sin \theta_{k}}+\frac{m_{e_{s}} V_{4 \ell}}{\sqrt{2} m_{W} \cos \theta_{k}}\right) \gamma_{L}\right] e_{s} \tilde{\nu}_{R s}^{*}\right\},
\end{aligned}
$$

where $\gamma_{L, R}=\left(1 \mp \gamma_{5}\right) / 2$ and

$$
\begin{aligned}
O_{i j}^{L} & =V_{2 i}^{*} V_{2 j}+V_{3 i}^{*} V_{3 j}+V_{4 i}^{*} V_{4 j}, \\
O_{i j}^{R} & =U_{2 i}^{*} U_{2 j}+U_{3 i}^{*} U_{3 j}+U_{4 i}^{*} U_{4 j} \\
O_{i j}^{\prime L} & =-V_{1 i}^{*} V_{1 j}-\frac{1}{2}\left(V_{3 i}^{*} V_{3 j}+V_{4 i}^{*} V_{4 j}\right)+\delta_{i j} \sin ^{2} \theta_{W}, \\
O_{i j}^{\prime R} & =-U_{1 i} U_{1 j}^{*}-\frac{1}{2}\left(U_{3 i} U_{3 j}^{*}+U_{4 i} U_{4 j}^{*}\right)+\delta_{i j} \sin ^{2} \theta_{W} .
\end{aligned}
$$

Thus, using Eqs. (5.60) and (5.61) the basic coupling vertices of the interaction Lagrangian (8.152) and the so called bilinear and quartic charges determining the cross-section-type physical observables, can be entirely written in terms of reduced projectors and eigenphases.

For instance, using Eqs. (5.60) and (5.61) we can write the coupling (8.153-8.156) in terms of the eigenphases and reduced projectors, namely

$$
\begin{aligned}
O_{i j}^{L} & =\zeta_{i} \zeta_{j}^{*} \sqrt{P_{i 11}^{V} P_{j 11}^{V}}\left(p_{i 2} p_{j 2}^{*}+\delta_{i 3} \delta_{j 3}+p_{i 4} p_{j 4}^{*}\right), \\
O_{i j}^{R} & =\sqrt{P_{i 11}^{U^{*}} P_{j 11}^{U^{*}}}\left(\tilde{p}_{i 2}^{*} \tilde{p}_{j 2}+\tilde{p}_{i 3}^{*} \tilde{p}_{j 3}+\delta_{i 3} \delta_{j 3}\right), \\
O_{i j}^{\prime L} & =\delta_{i j} \sin ^{2} \theta_{W}-\zeta_{i} \zeta_{j}^{*} \sqrt{P_{i 11}^{V} P_{j 11}^{V}}\left[\left(1-\delta_{i 3}\right)\left(1-\delta_{j 3}\right)+\frac{1}{2}\left(\delta_{i 3} \delta_{j 3}+p_{i 4} p_{j 4}^{*}\right)\right], \\
O_{i j}^{\prime} R & =\delta_{i j} \sin ^{2} \theta_{W}-\sqrt{P_{i 11}^{U^{*}} P_{j 11}^{U^{*}}}\left[\left(1-\delta_{i 3}\right)\left(1-\delta_{j 3}\right)+\frac{1}{2}\left(\delta_{i 3} \delta_{j 3}+\tilde{p}_{i 3} \tilde{p}_{j 3}^{*}\right)\right],
\end{aligned}
$$

where we have used the fact that $p_{i 3}=\tilde{p}_{i 4}=\delta_{i 3}, p_{i 1}=\tilde{p}_{i 1}=1-\delta_{i 3}$, and the choice $\tilde{\eta}_{j}=1, j=1, \ldots, 4$.

Note that when $i=j$, the coupling (8.157-8.160) are independent on the eigenphases, they only depends on the norm of the reduced projectors. On the other hand, when $i \neq j$, using Eqs. (5.62) and (5.63), we can show that these couplings in addition to the explicit dependence on the eigenphases, only depends on the norm of four $V$-type and four $U^{*}$-type reduced projectors.

Let us write $p_{i j}$ in the form

$$
p_{i j}=\left|p_{i j}\right| e^{i \beta_{i j}}
$$

where $\beta_{i j}$ is a real phase. Inserting this result into (5.62), and splitting the real and imaginary part, we get

$$
1+a_{i j}^{(2)} \cos \left(\beta_{i 2}-\beta_{j 2}\right)+a_{i j}^{(4)} \cos \left(\beta_{i 4}-\beta_{j 4}\right)=0
$$


and

$$
a_{i j}^{(2)} \sin \left(\beta_{i 2}-\beta_{j 2}\right)+a_{i j}^{(4)} \sin \left(\beta_{i 4}-\beta_{j 4}\right)=0,
$$

where $a_{i j}^{(k)}=\left|p_{i k}\right|\left|p_{j k}\right|, i, j=1, \ldots, 4,(i>j \perp i, j \neq 3)$. Solving these equations for the unknown $\beta_{i j}$ variables, we obtain

$$
\beta_{i 2}-\beta_{j 2}= \pm \arccos \left(X_{i j}\right)
$$

and

$$
\beta_{i 4}-\beta_{j 4}=\mp \arccos \left(Y_{i j}\right)
$$

where

$$
X_{i j}=\frac{-1+\left[\left(a_{i j}^{(4)}\right)^{2}-\left(a_{i j}^{(2)}\right)^{2}\right]}{2 a_{i j}^{(2)}}
$$

and

$$
Y_{i j}=\frac{-1-\left[\left(a_{i j}^{(4)}\right)^{2}-\left(a_{i j}^{(2)}\right)^{2}\right]}{2 a_{i j}^{(4)}}
$$

when $i>j \perp i, j \neq 3$.

The same relations are valid for the norm and phases of the $U^{*}$-type reduced projectors. Thus, according to Eqs. (8.164) and (8.165) (and the corresponding ones for the $U^{*}$-type reduced projectors) all phase differences in (8.157-8.160) only depends on the norm of the reduced projectors.

Moreover, Eq. (8.164) allow us eliminate two phases and obtain a real algebraic equations relating the norm of the reduced projectors, namely

$$
\begin{aligned}
& \beta_{22}=\beta_{12} \pm \arccos X_{21}, \\
& \beta_{42}=\beta_{12} \pm \arccos X_{41}
\end{aligned}
$$

and

$$
\arccos X_{21}-\arccos X_{41}+\arccos X_{42}=0 .
$$

In the same way, from Eq. (8.165), we get

$$
\begin{aligned}
& \beta_{24}=\beta_{14} \mp \arccos Y_{21}, \\
& \beta_{44}=\beta_{14} \mp \arccos Y_{41}
\end{aligned}
$$

and

$$
\arccos Y_{21}-\arccos Y_{41}+\arccos Y_{42}=0 .
$$

Note that from Eq. (5.63), analogous equations for the phases and norms of the reduced projectors of the $U^{*}$-type can be obtained by defining $\tilde{p}_{i j}=\left|\tilde{p}_{i j}\right| e^{i \tilde{\beta}_{i j}}, \tilde{a}_{i j}^{(k)}=\left|\tilde{p}_{i k}\right|\left|\tilde{p}_{j k}\right|, k=2,3, i, j=1, \ldots, 4,(i>$ $j \perp i, j \neq 3)$.

Thus, from Eqs. (8.168-8.173), and the analogous ones for the $U^{*}$-type reduced projectors, we deduce that the number of independent parameters determining the complete set of reduced projectors is twelve. The independent parameters can be chosen to be $\left|p_{12}\right|,\left|p_{22}\right|,\left|p_{14}\right|,\left|p_{24}\right|,\left|\tilde{p}_{12}\right|,\left|\tilde{p}_{22}\right|,\left|\tilde{p}_{13}\right|,\left|\tilde{p}_{23}\right|, \beta_{12}, \beta_{14}, \tilde{\beta}_{12}, \tilde{\beta}_{13}$. 
Moreover, using Eqs. (5.68-5.75), the independent phases can be expressed in terms of the fundamental L-R SUSY parameters and physical masses as

$$
\tan \beta_{1 j}=\frac{\operatorname{Im}\left(p_{1 j}\right)}{\operatorname{Re}\left(p_{1 j}\right)}=\frac{\operatorname{Im}\left(\Delta_{j 1}^{*}\right)}{\operatorname{Re}\left(\Delta_{j 1}^{*}\right)}
$$

when $j=2,4$, and

$$
\tan \tilde{\beta}_{1 j}=\frac{\operatorname{Im}\left(\tilde{p}_{1 j}\right)}{\operatorname{Re}\left(\tilde{p}_{1 j}\right)}=\frac{\operatorname{Im}\left(\tilde{\Delta}_{j 1}^{*}\right)}{\operatorname{Re}\left(\tilde{\Delta}_{j 1}^{*}\right)}
$$

when $j=2,3$. Note that the independent phases are connected with the eigenphases through Eqs. (5.81) and (5.83), i.e., eventually we could also choose the eigenphases as independent parameters in place of them.

On the other hand, if two of the chargino physical masses are known, for instance $m_{\tilde{\chi}_{1,2}^{ \pm}}$, combining Eqs. (5.80) and (5.81) we get that both the $\zeta_{j}$ eigenphases, $j=1,2,4$, and $\tan \theta_{k}$ parameter can be expressed in terms of the above mentioned set of twelve independent parameters and two given chargino physical masses. Moreover, as $\zeta_{3}=-\mu /|\mu|$, with the help of Eq. (5.79) when $j=1$ or $j=2$, we can also express the $\zeta_{3}$ eigenphase in terms of this set of parameters and physical masses. In this way, we conclude that all the L-R SUSY fundamental parameters can be expressed in terms of above mentioned set of independent parameters and physical masses.

In sum, when two chargino physical masses are known, the quartic charges determining the crosssection-type observables, in addition to the known chargino masses, depend on a subset of reduced projector norms and phases. Due to the large number of independent parameters we need to determine (twelve, if two physical chargino masses are known, or ten, if four physical chargino masses are known), the problem for extracting some information on the fundamental L-R SUSY parameters by measuring this class of observables is complex. Comparing with the corresponding case in the MSSM, in similar circumstances, the quartic charges only depends on two independent parameters[23, 24], in our approach they can be taken to be the norm of a $U^{*}$-type reduced projector and the one of a $V$-type reduced projector.

Returning to the counting of independent variables, in an idealized situation where all the physical chargino masses are known we need to determine ten parameters corresponding exactly to the number of independent equations obtained by measuring polarized or unpolarized chargino pair production total cross section $\sigma_{T}: e^{+} e^{-} \rightarrow \tilde{\chi}_{i}^{+} \tilde{\chi}_{j}^{+}, i, j=1, \ldots, 4 ; i \geq j$. The same occurs in the context of the MSSM where the number of independent parameters is two (when the two lightest chargino masses are known). Further independent relations as forward-backward asymmetries in the case of unpolarized beams or leftright asymmetries in the case of polarized beams could constitute a fundamental complement to fix all unknown reduced projectors, i.e., the unknown L-R SUSY parameters, up to a discrete ambiguity. Moreover, as in the MSSM [23], this discrete ambiguity could be resolved by analyzing manifestly CP-non invariant observables such as those related to normal polarization of the charginos or T-odd asymmetries related to the chargino pair production with longitudinally polarized beams and subsequent decays of one of the charginos into a sneutrino and anti-lepton. Evidently, from both the analytical and numerical point of view the situation is not evident. 
In the next section, we propose a suitable parametrization for the independent reduced projectors, i.e., basic couplings and cross-section-type physical observables that could help us to visualize the solution to our problem in a more excellent manner.

\subsection{Parametrization in spherical coordinates}

The particular choice of the set of independent reduced projectors determining the structure of the $V$ and $U^{*}$ matrices allows us to use spherical coordinates to parameterize the physical cross section observables. Indeed, from Eq. (5.60) and the unitary character of the $V$ matrix, we have

$$
P_{j 11}^{V}\left(1+\left|p_{j 2}\right|^{2}+\left|p_{j 4}\right|^{2}\right)=1, \quad j=1,2 .
$$

Then, we can define $(j=1,2)$

$$
\begin{aligned}
\sin \theta^{(j)} \cos \phi^{(j)} & =\sqrt{P_{j 11}^{V}}\left|p_{j 2}\right|, \\
\sin \theta^{(j)} \sin \phi^{(j)} & =\sqrt{P_{j 11}^{V}}\left|p_{j 4}\right|, \\
\cos \theta^{(j)} & =\sqrt{P_{j 11}^{V}},
\end{aligned}
$$

where $0 \leq \theta^{(j)} \leq \pi / 2$ and $0 \leq \phi^{(j)} \leq \pi$.

In the same way, from Eq. (5.61) and the unitary character of the $U^{*}$ matrix, we have

$$
P_{j 11}^{U^{*}}\left(1+\left|\tilde{p}_{j 2}\right|^{2}+\left|\tilde{p}_{j 3}\right|^{2}\right)=1, \quad j=1,2 .
$$

Then, again we can define $(j=1,2)$

$$
\begin{aligned}
\sin \tilde{\theta}^{(j)} \cos \tilde{\phi}^{(j)} & =\sqrt{P_{j 11}^{U^{*}}}\left|\tilde{p}_{j 2}\right|, \\
\sin \tilde{\theta}^{(j)} \sin \tilde{\phi}^{(j)} & =\sqrt{P_{j 11}^{U^{*}}}\left|\tilde{p}_{j 3}\right|, \\
\cos \tilde{\theta}^{(j)} & =\sqrt{P_{j 11}^{U^{*}}},
\end{aligned}
$$

where $0 \leq \tilde{\theta}^{(j)} \leq \pi / 2$ and $0 \leq \tilde{\phi}^{(j)} \leq \pi$

Thus, all independent reduced projector are expressed in terms of the spherical coordinated in the form $(j=1,2)$

$$
\begin{aligned}
\left|p_{j 2}\right| & =\tan \theta^{(j)} \cos \phi^{(j)}, & & \left|p_{j 4}\right|=\tan \theta^{(j)} \sin \phi^{(j)} \\
\left|\tilde{p}_{j 2}\right| & =\tan \tilde{\theta}^{(j)} \sin \tilde{\phi}^{(j)}, & \left|\tilde{p}_{j 3}\right| & =\tan \tilde{\theta}^{(j)} \cos \tilde{\phi}^{(j)} .
\end{aligned}
$$

The norms $\left|p_{42}\right| \equiv \sqrt{X}$ and $\left|p_{44}\right| \equiv \sqrt{Y}$, are obtained by solving the following system of equations deduced from Eqs. (8.170) and (8.173):

$$
\begin{gathered}
a_{20} X^{2}+a_{11} X Y+a_{02} Y^{2}+a_{10} X+a_{01} Y+a_{00}=0 \\
b_{20} X^{2}+b_{11} X Y+b_{02} Y^{2}+b_{10} X+b_{01} Y+b_{00}=0
\end{gathered}
$$


where the $a_{\alpha \beta}$ coefficients, $\alpha, \beta=1,2$ are given by

$$
\begin{aligned}
& a_{20}=\frac{1}{4}\left\{1+\cos ^{2} \phi^{(1)} \tan ^{2} \theta^{(1)}+\frac{1}{2} \tan ^{2} \theta^{(2)}\right. \\
& \left.\times \quad\left[\left(\cos \left(2 \phi^{(1)}\right)+\cos \left(2 \phi^{(2)}\right)\right) \sec ^{2} \theta^{(1)}+2 \sin ^{2} \phi^{(1)}\right]\right\}, \\
& a_{11}=\frac{1}{4}\left\{-\sin ^{2} \phi^{(2)} \tan ^{2} \theta^{(2)}\left(2+\cos ^{2} \phi^{(1)} \tan ^{2} \theta^{(1)}\right)\right. \\
& -\tan ^{2} \phi^{(1)}-\tan ^{2} \phi^{(2)}+\sin ^{2} \phi^{(1)} \tan ^{2} \theta^{(1)} \\
& \left.\times\left[-2+\tan ^{2} \theta^{(2)}\left(-\cos ^{2} \phi^{(2)}+\sin ^{2} \phi^{(2)}\left(\tan ^{2} \phi^{(1)}+\tan ^{2} \phi^{(2)}\right)\right)\right]\right\}, \\
& a_{02}=\frac{1}{8}\left\{2 \sin ^{2} \phi^{(1)} \tan ^{2} \theta^{(1)} \tan ^{2} \phi^{(1)}+\left[2 \sin ^{2} \phi^{(2)} \tan ^{2} \theta^{(2)}\right.\right. \\
& \left.\left.+\left(2+\left(\cos \left(2 \phi^{(1)}\right)+\cos \left(2 \phi^{(2)}\right)\right) \tan ^{2} \theta^{(1)} \tan ^{2} \theta^{(2)}\right) \tan ^{2} \phi^{(1)}\right] \tan ^{2} \phi^{(2)}\right\}, \\
& a_{10}=\frac{1}{16}\left\{\operatorname { c o t } ^ { 2 } \theta ^ { ( 1 ) } \operatorname { c o t } ^ { 2 } \theta ^ { ( 2 ) } \operatorname { s e c } ^ { 2 } \phi ^ { ( 1 ) } \operatorname { s e c } ^ { 2 } \phi ^ { ( 2 ) } \left[2+2 \cos ^{2} \phi^{(1)} \tan ^{2} \theta^{(1)}\right.\right. \\
& \left.+\left((\cos (2 \phi(1))+\cos (2 \phi(2))) \sec ^{2} \theta^{(1)}+2 \sin ^{2} \phi^{(1)}\right) \tan ^{2} \theta^{(2)}\right] \\
& \left.\times\left[2+\left(\cos \left(2 \phi^{(1)}\right)+\cos \left(2 \phi^{(2)}\right)\right) \tan ^{2} \theta^{(1)} \tan ^{2} \theta^{(2)}\right]\right\}, \\
& a_{01}=\frac{1}{8}\left\{\left[-4-\sec ^{2} \phi^{(2)}\left(2 \cot ^{2} \theta^{(2)}+\left(\cos \left(2 \phi^{(1)}\right)+\cos \left(2 \phi^{(2)}\right)\right) \tan ^{2} \theta^{(1)}\right)\right] \tan ^{2} \phi^{(1)}\right. \\
& \left.-\left[4+\sec ^{2} \phi^{(1)}\left(2 \cot ^{2} \theta^{(1)}+\left(\cos \left(2 \phi^{(1)}\right)+\cos \left(2 \phi^{(2)}\right)\right) \tan ^{2} \theta^{(2)}\right)\right] \tan ^{2} \phi^{(2)}\right\},
\end{aligned}
$$

and

$$
a_{00}=\frac{1}{4}\left\{\left(1+\cot ^{2} \theta^{(1)} \sec ^{2} \phi^{(1)}\right)\left(1+\cot ^{2} \theta^{(2)} \sec ^{2} \phi^{(2)}\right)-\tan ^{2} \phi^{(1)} \tan ^{2} \phi^{(2)}\right\},
$$

and the $b_{\alpha \beta}$ coefficients are obtained from the corresponding $a_{\beta \alpha}$ by replacing $\sin \phi^{(j)}$ by $\cos \phi^{(j)}, j=1,2$ or vice versa, in all the terms containing these functions in either the explicit or implicit form.

Similarly, the norms $\left|\tilde{p}_{42}\right| \equiv \sqrt{\tilde{X}}$ and $\left|\tilde{p}_{43}\right| \equiv \sqrt{\tilde{Y}}$, are obtained by solving the following equation system:

$$
\begin{gathered}
\tilde{a}_{20} \tilde{X}^{2}+\tilde{a}_{11} \tilde{X} \tilde{Y}+\tilde{a}_{02} \tilde{Y}^{2}+\tilde{a}_{10} \tilde{X}+\tilde{a}_{01} \tilde{Y}+\tilde{a}_{00}=0 \\
\tilde{b}_{20} \tilde{X}^{2}+\tilde{b}_{11} \tilde{X} \tilde{Y}+\tilde{b}_{02} \tilde{Y}^{2}+\tilde{b}_{10} \tilde{X}+b_{01} \tilde{Y}+\tilde{b}_{00}=0
\end{gathered}
$$

where the $\tilde{a}_{\alpha \beta}$ and $\tilde{b}_{\alpha \beta}$ coefficients, $\alpha, \beta=1,2$ are the same as the $a_{\alpha \beta}$ and $b_{\alpha \beta}$ coefficients, respectively, with the obvious changes. 
Note that from the geometric point of view, the independent reduced projector-type parameters lie on four disconnected spherical surfaces and the dependent ones are determined by intersecting two conical sections.

Thus, for instance, from Eqs. (8.153-8.156), we deduce that the coupling constants in spherical coordinates are given by

$$
\begin{aligned}
O_{i j}^{L} & =\delta_{i j}-\zeta_{i} \zeta_{j}^{*} \cos \theta^{(i)} \cos \theta^{(j)} \\
O_{i j}^{R} & =\delta_{i j}-\cos \tilde{\theta}^{(i)} \cos \tilde{\theta}^{(j)}, \\
O_{i j}^{\prime L} & =\delta_{i j} \sin ^{2} \theta_{W}-\zeta_{i} \zeta_{j}^{*} \cos \theta^{(i)} \cos \theta^{(j)} \\
& \times\left\{1+\frac{1}{2} \tan \theta^{(i)} \tan \theta^{(j)} \sin \phi^{(i)} \sin \phi^{(j)}\left[Y_{i j} \mp \mathbf{i} \sqrt{1-Y_{i j}^{2}}\right]\right\}, \\
O_{i j}^{\prime L} & =\delta_{i j} \sin ^{2} \theta_{W}-\cos \tilde{\theta}^{(i)} \cos \tilde{\theta}^{(j)} \\
& \times\left\{1+\frac{1}{2} \tan \tilde{\theta}^{(i)} \tan \theta^{(j)} \sin \tilde{\phi}^{(i)} \sin \tilde{\phi}^{(j)}\left[\tilde{Y}_{i j} \mp \mathbf{i} \sqrt{1-\tilde{Y}_{i j}^{2}}\right]\right\},
\end{aligned}
$$

when $i, j=1,2$, and

$$
O_{3 j}^{L}=O_{j 3}^{L}=O_{3 j}^{R}=O_{j 3}^{R}=\delta_{3 j}, \quad O_{3 j}^{L}=O_{j 3}^{\prime L}=O_{3 j}^{\prime R}=O_{j 3}^{\prime R}=\delta_{3 j}\left(\sin ^{2} \theta_{W}-\frac{1}{2}\right),
$$

when $j=1,2,3$. Here,

$$
Y_{i j}=\frac{-2+\left[\cos \left(2 \phi^{(i)}\right)+\cos \left(2 \phi^{(j)}\right)\right] \tan ^{2} \theta^{(i)} \tan ^{2} \theta^{(j)}}{4 \tan \theta^{(i)} \tan \theta^{(j)} \sin \phi^{(i)} \sin \phi^{(j)}}
$$

and $Y_{i i} \equiv 1$, with analogous expressions for $\tilde{Y}_{i j}$. As we have pointed out, when the two lightest chargino masses are known, from Eq. (5.80) we can express the fundamental parameter $\tan \theta_{k}$ in terms of these masses and the independent reduced projector-type parameters, i.e., in terms of the two lightest chargino masses, the spherical angles defined in this section and the four independent reduced projector phases, see Appendix B. Moreover, using Eq. (5.81), $\zeta_{j}, j=1,2$, can also be expressed in terms of this set of parameters. Hence, in a first stage, we should analyze those observables that depend directly on these parameters.

On the other hand, when either $i=4$ or $j=4$, the couplings in Eqs. (8.153-8.156) in addition to the basic independent parameters depend on the norms $\left|p_{42}\right|,\left|p_{44}\right|,\left|\tilde{p}_{42}\right|,\left|\tilde{p}_{43}\right|$, i.e., we must solve Eqs. (8.186-8.187) and Eqs. (8.194-8.195) to obtain the explicit dependence of these coupling in terms of the physical chargino masses and the complete set of angular independent parameters. From both, the analytical and numerical point of view, the determination of the independent parameters through the experimental measurements of observables containing these dependent terms is more complex.

\section{Conclusions}

In this paper we have studied the consequences produced by the introduction of $\mathrm{CP}$-phases into the chargino mass matrix in the context of the L-R SUSY model. We have analyzed the chargino mass spectrum and treated the inverse parameter problem. Thus, the chargino mass matrix was described by eight real 
fundamental parameters, i.e., the four usual parameters $\left|M_{L}\right|,|\mu|, M_{R}$, and $\tan \theta_{k}$ in addition to the four real phases $\Phi_{L}, \Phi_{\mu}, \tilde{\Phi}_{L}$ and $\tilde{\Phi}_{R}$. To find analytical expressions for the chargino physical masses $m_{\tilde{\chi}_{j}^{ \pm}}, j=1, \ldots, 4$, and some connecting relations among the parameters, at the tree level, we have diagonalized the non-symmetric chargino mass matrix by constructing two diagonalizing unitary matrices. The masses, obtained by solving the associated characteristic polynomial to this problem, have been ordered by sizes and plotted as a function of the Higgsino parameter $\mu$, and also as a function of $\tan \theta_{k}$ by considering some possible $\mathrm{CP}$-conserving and CP-violating scenarios. We have observed by comparing different plots that the effects on the mass spectrum are more significative when the mixing phases $\tilde{\Phi}_{L}$ or $\Phi_{L}$ varies. Some comparisons with the MSSM have been given.

The inverse problem consisting to determine the fundamental parameters in terms of the chargino physical masses, the reduced projectors and the eigenphases have been solved using the projector formalism based on the construction of the two mentioned diagonalizing matrices of this problem. Also, combining these results with the corresponding Jarlskog's formulas we have obtained an alternative way to disentangle the unknown L-R SUSY parameters. In particular, under some conditions, we have found analytic expressions to disentangle the parameters $\left|M_{L}\right|, \Phi_{L}, \tilde{\Phi}_{L}$, from the rest of the parameters. Thus, we have considered three types of CP-violating scenarios, all of them characterized by a big rate between $k_{u}$ and $k_{d}$. In one of these scenarios we have supposed that the quantities that could be first measured are the two lightest chargino masses and the two lightest neutralino masses. In this case, we have observed that analytical and numerical expressions can be given which allow us disentangle the parameters $\left|M_{L}\right|, \Phi_{L}$ and $\tilde{\Phi}_{L}$ up to a twofold discrete ambiguity. Also, an additional equation should allows us to extract, at least numerically, an additional parameter.

In a more general schema, we have demonstrated that the fundamental L-R SUSY parameters can be expressed in terms of twelve independent parameters associated to the reduced projectors, which can be represented by four pair of spherical angles an four independent reduced projector phases. The analytical or numerical determination of these parameters through the measurement of cross-section-type observables could allow us, in principle, to known all the fundamental L-R SUSY parameters. However, the treatment of this problem is not simple since the large number of involved parameters difficult its resolution. A possible issue to the problem could be to give some appropriate physical inputs in the chargino and neutralino sectors.

The formulas deduced in this article allow us to considerer other possibilities of input data. For instance, some scenarios where the lightest chargino and neutralino masses are known. Also, as the basic system of equations given in Eqs. (5.66) and (5.67) only involve the matrix elements of the original chargino mass matrix, the projectors, the reduced projectors, the chargino physical masses and the eigenphases, and these equations remains uncoupled with respect to the index $j$, the present formalism can be directly generalized to any chargino number and any complex non-symmetric mass matrix. For instance, the inverse problem for determining the fundamental parameters including all terms of the Lagrangian (2.8) can be treated in the same way, i.e., we could determine the contribution of the charged right-handed higgsino fields $\tilde{\Delta}_{R}$ and $\tilde{\delta}_{R}$ to the determination of the fundamental parameters. However, in this case, to determine the unknown physical chargino masses in terms of the fundamental parameters we must solve a quintic equation which requires some additional work. These last aspects could be treated in a separate communication. 


\section{Acknowledgments}

The author dedicates this article to the memory of his friend Jeff Delbecque, Celui qui savait paler aux femmes. He thank Patricia Aguilar, member of the organization Baobab Familial of Montreal, for valuable support, Artorix de la Cruz de Oña and Mariana Frank for enlightening discussions, and the referees for valuable lecture and suggestions in order to enhance the presentation and contents of this paper.

\section{A Chargino mass spectrum}

According to Eq. (3.25), the chargino masses predicted by the present model are given by the positive roots of the eigenvalues associated to either the Hermitian matrix $H \equiv M^{\dagger} M$ or the Hermitian matrix $\tilde{H} \equiv M M^{\dagger}$. These eigenvalues can be obtained by solving the common characteristic equation associated to these matrices. Indeed, starting from Eq. (3.25), we get

$$
\left(M^{\dagger} M\right) V-V M_{D}^{2}=0
$$

and

$$
\left(M M^{\dagger}\right) U^{*}-U^{*} M_{D}^{2}=0
$$

which expressed by components writes

$$
\left(H_{i i}-m_{\tilde{\chi}_{j}^{ \pm}}^{2}\right) V_{i j}+\sum_{k \neq i}^{4} H_{i k} V_{k j}, \quad i, j=1, \ldots, 4,
$$

and

$$
\left(\tilde{H}_{i i}-m_{\tilde{\chi}_{j}^{ \pm}}^{2}\right) U_{i j}^{*}+\sum_{k \neq i}^{4} \tilde{H}_{i k} U_{k j}^{*}, \quad i, j=1, \ldots, 4,
$$

respectively, where $H_{i j}=\sum_{k=1}^{4} M_{k i}^{*} M_{k j}$ :

$$
\begin{aligned}
H_{11} & =\left|M_{L}\right|^{2}+2\left|\tilde{M}_{L}\right|^{2} \sin ^{2} \theta_{k}, \\
H_{22} & =M_{R}^{2}+2\left|\tilde{M}_{R}\right|^{2} \sin ^{2} \theta_{k}, \\
H_{33} & =|\mu|^{2} \\
H_{44} & =|\mu|^{2}+2\left(\left|\tilde{M}_{L}\right|^{2}+\left|\tilde{M}_{R}\right|^{2}\right) \cos ^{2} \theta_{k}, \\
H_{12} & =H_{21}^{*}=2\left|\tilde{M}_{L}\right|\left|\tilde{M}_{R}\right| e^{i\left(\tilde{\Phi}_{R}-\tilde{\Phi}_{L}\right)} \sin ^{2} \theta_{k}, \\
H_{13} & =H_{31}^{*}=0 \\
H_{14} & =H_{41}^{*}=\sqrt{2}\left|\tilde{M}_{L}\right|\left[e^{i\left(\tilde{\Phi}_{L}-\Phi_{L}\right)}\left|M_{L}\right| \cos \theta_{k}\right. \\
& \left.-e^{i\left(\Phi_{\mu}-\tilde{\Phi}_{L}\right)}|\mu| \sin \theta_{k}\right] \\
H_{23} & =H_{32}^{*}=0, \\
H_{24} & =H_{42}^{*}=\sqrt{2}\left|\tilde{M}_{R}\right|\left[e^{i \tilde{\Phi}_{R}} M_{R} \cos \theta_{k}\right. \\
& \left.-e^{i\left(\Phi_{\mu}-\tilde{\Phi}_{R}\right)}|\mu| \sin \theta_{k}\right] \\
H_{34} & =H_{43}^{*}=0 .
\end{aligned}
$$


and $\tilde{H}_{i j}=\sum_{k=1}^{4} M_{i k} M_{j k}^{*}$ :

$$
\begin{aligned}
\tilde{H}_{11} & =\left|M_{L}\right|^{2}+2\left|\tilde{M}_{L}\right|^{2} \cos ^{2} \theta_{k}, \\
\tilde{H}_{22} & =M_{R}^{2}+2\left|\tilde{M}_{R}\right|^{2} \cos ^{2} \theta_{k}, \\
\tilde{H}_{33} & =|\mu|^{2}+2\left(\left|\tilde{M}_{L}\right|^{2}+\left|\tilde{M}_{R}\right|^{2}\right) \sin ^{2} \theta_{k}, \\
\tilde{H}_{44} & =|\mu|^{2}, \\
\tilde{H}_{12} & =\tilde{H}_{21}^{*}=2\left|\tilde{M}_{L}\right|\left|\tilde{M}_{R}\right| e^{i\left(\tilde{\Phi}_{L}-\tilde{\Phi}_{R}\right)} \cos ^{2} \theta_{k}, \\
\tilde{H}_{13} & =\tilde{H}_{31}^{*}=\sqrt{2}\left|\tilde{M}_{L}\right|\left[e^{i\left(\Phi_{L}-\tilde{\Phi}_{L}\right)}\left|M_{L}\right| \sin \theta_{k}\right. \\
& \left.-e^{i\left(\tilde{\Phi}_{L}-\Phi_{\mu}\right)}|\mu| \cos \theta_{k}\right], \\
\tilde{H}_{14} & =\tilde{H}_{41}^{*}=0, \\
\tilde{H}_{23} & =\tilde{H}_{32}^{*}=\sqrt{2}\left|\tilde{M}_{R}\right|\left[e^{-i \tilde{\Phi}_{R}} M_{R} \sin \theta_{k}\right. \\
& \left.-e^{i\left(\tilde{\Phi}_{R}-\Phi_{\mu}\right)}|\mu| \cos \theta_{k}\right], \\
\tilde{H}_{24} & =\tilde{H}_{42}^{*}=0, \\
\tilde{H}_{34} & =\tilde{H}_{43}^{*}=0 .
\end{aligned}
$$

For fixed $j$, each of the Eqs. (A.3) and (A.4) represents a system of homogeneous linear equations depending on only one of the chargino masses. Thus, the chargino masses can be determined by solving the characteristic equation associated to these systems, that is

$$
X^{4}-a X^{3}+b X^{2}-c X+d=0
$$

where

$$
\begin{gathered}
a=\left|M_{L}\right|^{2}+M_{R}^{2} \\
+2\left(\left|\tilde{M}_{L}\right|^{2}+\left|\tilde{M}_{R}\right|^{2}+|\mu|^{2}\right) \\
b=|\mu|^{4}+2|\mu|^{2}\left(\left|M_{L}\right|^{2}+M_{R}^{2}+\left|\tilde{M}_{L}\right|^{2}+\left|\tilde{M}_{R}\right|^{2}\right) \\
+2|\mu| \sin \left(2 \theta_{k}\right)\left[\left|M_{L}\right|\left|\tilde{M}_{L}\right|^{2} \cos \left(\Phi_{L}-2 \tilde{\Phi}_{L}+\Phi_{\mu}\right)\right. \\
\left.+\quad M_{R}\left|\tilde{M}_{R}\right|^{2} \cos \left(2 \tilde{\Phi}_{R}-\Phi_{\mu}\right)\right]+2 M_{R}^{2}\left|\tilde{M}_{L}\right|^{2} \\
+\left|M_{L}\right|^{2}\left(M_{R}^{2}+2\left|\tilde{M}_{R}\right|^{2}\right) \\
+\left(\left|\tilde{M}_{L}\right|^{2}+\left|\tilde{M}_{R}\right|^{2}\right)^{2} \sin ^{2}\left(2 \theta_{k}\right),
\end{gathered}
$$




$$
\begin{aligned}
c & =|\mu|^{4}\left(M_{R}^{2}+\left|M_{L}\right|^{2}\right)+2|\mu|^{3} \sin \left(2 \theta_{k}\right) \\
& \times\left[\left|M_{L}\right|\left|\tilde{M}_{L}\right|^{2} \cos \left(\Phi_{L}-2 \tilde{\Phi}_{L}+\Phi_{\mu}\right)\right. \\
& \left.+M_{R}\left|\tilde{M}_{R}\right|^{2} \cos \left(2 \tilde{\Phi}_{R}-\Phi_{\mu}\right)\right]+|\mu|^{2} \\
& \times\left[\left(\left|\tilde{M}_{L}\right|^{2}+\left|\tilde{M}_{R}\right|^{2}\right)^{2} \sin ^{2}\left(2 \theta_{k}\right)+2\left|M_{L}\right|^{2}\left|\tilde{M}_{R}\right|^{2}\right. \\
& \left.+2 M_{R}^{2}\left(\left|M_{L}\right|^{2}+\left|\tilde{M}_{L}\right|^{2}\right)\right]+2|\mu|\left|M_{L}\right| M_{R} \sin \left(2 \theta_{k}\right) \\
& \times\left[M_{R}\left|\tilde{M}_{L}\right|^{2} \cos \left(\Phi_{L}-2 \tilde{\Phi}_{L}+\Phi_{\mu}\right)\right. \\
& \left.+\left|M_{L}\right|\left|\tilde{M}_{R}\right|^{2} \cos \left(2 \tilde{\Phi}_{R}-\Phi_{\mu}\right)\right]+\sin ^{2}\left(2 \theta_{k}\right) \\
& \times\left[2 M_{R}\left|M_{L}\right|\left|\tilde{M}_{L}\right|^{2}\left|\tilde{M}_{R}\right|^{2} \cos \left(\Phi_{L}-2 \tilde{\Phi}_{L}+2 \tilde{\Phi}_{R}\right)\right. \\
& \left.+M_{R}^{2}\left|\tilde{M}_{L}\right|^{4}+\left|M_{L}\right|^{2}\left|\tilde{M}_{R}\right|^{4}\right]
\end{aligned}
$$

and

$$
\begin{aligned}
d & =|\mu|^{2}\left\{\operatorname { s i n } ^ { 2 } ( 2 \theta _ { k } ) \left[M_{R}^{2}\left|\tilde{M}_{L}\right|^{4}+\left|M_{L}\right|^{2}\left|\tilde{M}_{R}\right|^{4}\right.\right. \\
& \left.+2\left|M_{L}\right| M_{R}\left|\tilde{M}_{L}\right|^{2}\left|\tilde{M}_{R}\right|^{2} \cos \left(\Phi_{L}-2 \tilde{\Phi}_{L}+2 \tilde{\Phi}_{R}\right)\right] \\
& +2 M_{R}|\mu|\left|M_{L}\right| \sin \left(2 \theta_{k}\right) \\
& \times\left[M_{R}\left|\tilde{M}_{L}\right|^{2} \cos \left(\Phi_{L}-2 \tilde{\Phi}_{L}+\Phi_{\mu}\right)\right. \\
& \left.\left.+\left|M_{L}\right|\left|\tilde{M}_{R}\right|^{2} \cos \left(2 \tilde{\Phi}_{R}-\Phi_{\mu}\right)\right]+\left|M_{L}\right|^{2} M_{R}^{2}|\mu|^{2}\right\} .
\end{aligned}
$$

Solving Eq. (A.7), we get the analytic formulas for the chargino masses

$$
\begin{aligned}
& m_{\tilde{\chi}_{1}^{ \pm}}^{2}, m_{\tilde{\chi}_{2}^{ \pm}}^{2}=\frac{a}{4}-\frac{\alpha}{2} \mp \frac{1}{2} \sqrt{\beta-\varpi-\frac{\lambda}{4 \alpha}}, \\
& m_{\tilde{\chi}_{3}^{ \pm}}^{2}, m_{\tilde{\chi}_{4}^{ \pm}}^{2}=\frac{a}{4}+\frac{\alpha}{2} \mp \frac{1}{2} \sqrt{\beta-\varpi+\frac{\lambda}{4 \alpha}},
\end{aligned}
$$

where

$$
\begin{aligned}
\alpha & =\sqrt{\frac{\beta}{2}+\varpi} \\
\varpi & =\frac{\epsilon}{32^{\frac{1}{3}}}+\frac{\left(2^{\frac{1}{3}} \gamma\right)}{3 \epsilon} \\
\epsilon & =\left(\delta+\sqrt{\delta^{2}-4 \gamma^{3}}\right)^{\frac{1}{3}} \\
\beta & =\frac{a^{2}}{2}-\frac{4 b}{3} \\
\lambda & =a^{3}-4 a b+8 c \\
\gamma & =b^{2}-3 a c+12 d, \\
\delta & =2 b^{3}-9 a b c+27 c^{2}+27 a^{2} d-72 b d
\end{aligned}
$$

We note that the physical masses have been ordered according to their increasing magnitude. Also, as $|\mu|^{2}$ is an exact root of Eq. (A.7), then it is always possible to find a neighborhood in the fundamental parameter space where one of the chargino physical masses take the value $|\mu|$. 


\section{B Disentangling $\tan \theta_{k}$}

When the two lightest physical chargino masses are known, from Eq. (5.80), we get

$$
f_{4} \tan ^{4} \theta_{k}+f_{3} \tan ^{3} \theta_{k}+f_{2} \tan ^{2} \theta_{k}+f_{1} \tan \theta_{k}+f_{0}=0,
$$

where

$$
\begin{aligned}
& f_{0}=\sin ^{2} \theta^{(1)} \sin ^{2} \theta^{(2)} \sin ^{2} \phi^{(1)} \sin ^{2} \phi^{(2)}\left\{m_{\tilde{\chi}_{1}^{ \pm}}^{2} \cos ^{2} \tilde{\phi}^{(2)} \cos ^{2} \phi^{(1)} \sin ^{2} \tilde{\theta}^{(2)} \sin ^{2} \theta^{(1)}\right. \\
& \left.-m_{\tilde{\chi}_{2}^{ \pm}}^{2} \cos ^{2} \tilde{\phi}^{(1)} \cos ^{2} \phi^{(2)} \sin ^{2} \tilde{\theta}^{(1)} \sin ^{2} \theta^{(2)}\right\} \text {, } \\
& f_{1}=2 \sin ^{2} \theta^{(1)} \sin ^{2} \theta^{(2)} \sin \phi^{(1)} \sin \phi^{(2)} \\
& \times\left\{\cos \left[\beta_{22}-\beta_{24}+\tilde{\beta}_{22}-\tilde{\beta}_{23}\right] \cos \tilde{\phi}^{(2)} \cos \phi^{(2)} \sin ^{2} \tilde{\theta}^{(2)} \sin \tilde{\phi}^{(2)} \sin \phi^{(1)}\right. \\
& \times\left(m_{\tilde{\chi}_{2}^{ \pm}}^{2} \cos ^{2} \tilde{\phi}^{(1)} \sin ^{2} \tilde{\theta}^{(1)}-m_{\tilde{\chi}_{1}^{ \pm}}^{2} \cos ^{2} \phi^{(1)} \sin ^{2} \theta^{(1)}\right)+\cos \left[\beta_{12}-\beta_{14}+\tilde{\beta}_{12}-\tilde{\beta}_{13}\right] \\
& \times \cos \tilde{\phi}^{(1)} \cos \phi^{(1)} \sin ^{2} \tilde{\theta}^{(1)} \sin \tilde{\phi}^{(1)} \sin \phi^{(2)} \\
& \left.\times\left(m_{\tilde{\chi}_{2}^{ \pm}}^{2} \cos ^{2} \phi^{(2)} \sin ^{2} \theta^{(2)}-m_{\tilde{\chi}_{1}^{ \pm}}^{2} \cos ^{2} \tilde{\phi}^{(2)} \sin ^{2} \tilde{\theta}^{(2)}\right)\right\}, \\
& f_{2}=m_{\tilde{\chi}_{1}^{ \pm}}^{2} \sin ^{2} \tilde{\theta}^{(2)} \sin ^{2} \theta^{(2)} \\
& \times\left[\cos ^{2} \tilde{\phi}^{(1)} \cos ^{2} \tilde{\phi}^{(2)} \sin ^{4} \tilde{\theta}^{(1)} \sin ^{2} \tilde{\phi}^{(1)} \sin ^{2} \phi^{(2)}\right. \\
& \left.+\cos ^{2} \phi^{(1)} \cos ^{2} \phi^{(2)} \sin ^{2} \tilde{\phi}^{(2)} \sin ^{4} \theta^{(1)} \sin ^{2} \phi^{(1)}\right] \\
& +4\left(m_{\tilde{\chi}_{1}^{ \pm}}^{2}-m_{\tilde{\chi}_{2}^{ \pm}}^{2}\right) \cos \left[\beta_{12}-\beta_{14}+\tilde{\beta}_{12}-\tilde{\beta}_{13}\right] \cos \left[\beta_{22}-\beta_{24}+\tilde{\beta}_{22}-\tilde{\beta}_{23}\right] \\
& \times \cos \tilde{\phi}^{(1)} \cos \tilde{\phi}^{(2)} \cos \phi^{(1)} \cos \phi^{(2)} \sin ^{2} \tilde{\theta}^{(1)} \sin ^{2} \tilde{\theta}^{(2)} \\
& \times \sin \tilde{\phi}^{(1)} \sin \tilde{\phi}^{(2)} \sin \phi^{(1)} \sin \phi^{(2)} \sin ^{2} \theta^{(1)} \sin ^{2} \theta^{(2)} \\
& \text { - } m_{\tilde{\chi}_{2}^{ \pm}}^{2} \sin ^{2} \tilde{\theta}^{(1)} \sin ^{2} \theta^{(1)} \\
& \times\left[\cos ^{2} \tilde{\phi}^{(1)} \cos ^{2} \tilde{\phi}^{(2)} \sin ^{4} \tilde{\theta}^{(2)} \sin ^{2} \tilde{\phi}^{(2)} \sin ^{2} \phi^{(1)}\right. \\
& \left.+\cos ^{2} \phi^{(1)} \cos ^{2} \phi^{(2)} \sin ^{2} \tilde{\phi}^{(1)} \sin ^{4} \theta^{(2)} \sin ^{2} \phi^{(2)}\right] \text {, } \\
& f_{3}=2 \sin ^{2} \tilde{\theta}^{(1)} \sin ^{2} \tilde{\theta}^{(2)} \sin \tilde{\phi}^{(1)} \sin \tilde{\phi}^{(2)}\left\{\cos \left[\beta_{22}-\beta_{24}+\tilde{\beta}_{22}-\tilde{\beta}_{23}\right]\right. \\
& \times \cos \tilde{\phi}^{(2)} \cos \phi^{(2)} \sin \tilde{\phi}^{(1)} \sin ^{2} \theta^{(2)} \sin \phi^{(2)} \\
& \times\left(m_{\tilde{\chi}_{2}^{ \pm}}^{2} \cos ^{2} \phi^{(1)} \sin ^{2} \theta^{(1)}-m_{\tilde{\chi}_{1}^{ \pm}}^{2} \cos ^{2} \tilde{\phi}^{(1)} \sin ^{2} \tilde{\theta}^{(1)}\right)+\cos \left[\beta_{12}-\beta_{14}+\tilde{\beta}_{12}-\tilde{\beta}_{13}\right] \\
& \times \cos \tilde{\phi}^{(1)} \cos \phi^{(1)} \sin \tilde{\phi}^{(2)} \sin ^{2} \theta^{(1)} \sin \phi^{(1)} \\
& \left.\times\left(m_{\tilde{\chi}_{2}^{ \pm}}^{2} \cos ^{2} \tilde{\phi}^{(2)} \sin ^{2} \tilde{\theta}^{(2)}-m_{\tilde{\chi}_{1}^{ \pm}}^{2} \cos ^{2} \phi^{(2)} \sin ^{2} \theta^{(2)}\right)\right\}
\end{aligned}
$$


and

$$
\begin{aligned}
f_{4} & =\sin ^{2} \tilde{\theta}^{(1)} \sin ^{2} \tilde{\theta}^{(2)} \sin ^{2} \tilde{\phi}^{(1)} \sin ^{2} \tilde{\phi}^{(2)}\left\{m_{\tilde{\chi}_{1}^{ \pm}}^{2} \cos ^{2} \tilde{\phi}^{(1)} \cos ^{2} \phi^{(2)} \sin ^{2} \theta^{(2)} \sin ^{2} \tilde{\theta}^{(1)}\right. \\
& \left.-m_{\tilde{\chi}_{2}^{ \pm}}^{2} \cos ^{2} \tilde{\phi}^{(2)} \cos ^{2} \phi^{(1)} \sin ^{2} \theta^{(1)} \sin ^{2} \tilde{\theta}^{(2)}\right\} .
\end{aligned}
$$

The four solutions of Eq. (B.15) are obtained from Eqs. (A.12-A.14) by putting

$$
a=-\frac{f_{3}}{f_{4}}, \quad b=\frac{f_{2}}{f_{4}}, \quad c=-\frac{f_{1}}{f_{4}}, \quad d=\frac{f_{0}}{f_{4}} .
$$

Note that $\tan \theta_{k}$ depend on the phases $\beta_{12}, \beta_{14}, \tilde{\beta}_{12}$ and $\tilde{\beta}_{13}$ through the phase differences $\beta_{12}-\beta_{14}$ and $\tilde{\beta}_{12}-\tilde{\beta}_{13}$.

\section{Summary of results}

In this section we summarize the principal relations among the parameters deduced from the chargino sector of the L-R SUSY model. Here, we don't display neither the results for the disentangled quantities obtained by considering the two lightest neutralino physical masses as input parameters or the disentangled expressions for $M_{R}$ and redefined eigenphases obtained as a consequence of introducing a novel parametrization. With respect to this last point, using this novel parametrization it is possible to think other scenarios starting with different sets of input parameters.

\section{References}

[1] R.M. Francis, M. Frank and C.S. Kalman, Phys. Rev. D 432369 (1991).

[2] H.N. Saif and C.S. Kalman, Z. Phys. C 56447 (1992).

[3] J. C. Pati and A. Salam, Phys. Rev. D 10275 (1974); R. N. Mohapatra and J. C. Pati, Phys. Rev. D 11 566, 2558 (1975); G. Senjanović and R. N. Mohapatra, Phys. Rev. D 121502 (1975); R. E. Marshak and R. N. Mohapatra, Phys. Lett. B 91222 (1980).

[4] M. Cvetic and J. Paty, Phys. Lett. B 13557 (1984)

[5] M. Frank and C. S. Kalman, Phys. Rev. D 38, 1469 (1988).

[6] R. Kuchimanchi and R. N. Mohapatra, Phys. Rev. D 484352 (1993).

[7] H. N. Saif, Mod. Phys. Lett. A 91131 (1994).

[8] K.Huitu, J. Maalampi, and M. Raidal, Phys. Lett. B 32860 (1994).

[9] K.Huitu, J. Maalampi, and M. Raidal, Nucl. Phys. B 420449 (1994).

[10] K. Huitu and J. Maalampi, preprint HU-SEFT R 1994-14. 
[11] N. Alvarez-Moraga and A. de la Cruz de Oña, Phys. Rev. D 72, 1 (2005).

[12] G. J. Gounaris, C. Le Mouël, and P. I. Porfyriadis, Phys. Rev. D 65, 035002 (2002).

[13] G. J. Gounaris and C. Le Mouël, Phys. Rev. D 66, 55007 (2002).

[14] H.P. Nilles, Phys. Rep. 100, 1 (1984); H.E. Haber and G. L. Kane, Phys. Rep. 117, 75 (1985).

[15] M. Frank, C. S. Kalman, and H. S. Saif, Z. Phys. C 59, 655 (1993).

[16] S. Y. Choi, M. Guchait, J. Kalinowski, and P. M. Zerwas, Phys. Lett. B479, 235 (2000).

[17] C. Jarlskog, Phys. Rev. D 362128 (1887); C. Jarlskog, Z. Phys. C 29, 491 (1985); C. Jarlskog, Stockholm Report USIP 87-14.

[18] M. Frank and H. S. Saif, Z. Phys. C 69, 673 (1996).

[19] R.N. Mohapatra, Supersymmetric Left Right Model, Automatic conservation and constraints on the $W_{R}$ Mass, ArXiv: hep-ph/9806520 (1998); C. S. Aulakh, A. Melfo, G. Senjanović, Phys. Rev. D 58, 115007 (1998).

[20] S. Y. Choi, A. Djouadi, H. S. Song, and P. M. Zerwas, Eur. Phys. J. C 8, 669 (1999).

[21] S. Y. Choi, J. Kalinowski, G. Moortgat-Pick, and P. M. Zerwas, Eur. Phys. J. C. 22, 563 (2001); arXiv:hep-ph/0108117 (2001).

[22] M. M. El Kleishen, A. A. Aboshousha, and A. A. Shafik, Phys. Rev D 45, 4345 (1992).

[23] S.Y. Choi, KIAS-P-99-094, hep-ph/9910325.

[24] J. L. Kneur and G. Moultaka, Phys. Rev. D 61, 095003 (2000); hep-ph/9907360.

[25] V. D. Barger, T. Han. T. J. Li, and T. Plehn, Phys. Lett. B 475, 342 (2000); arXiv:hep-ph/9907425. 